\title{
Recognizing Even-Cycle and Even-Cut Matroids
}

\author{
by \\ Cheolwon Heo
}

\author{
A thesis \\ presented to the University of Waterloo \\ in fulfillment of the \\ thesis requirement for the degree of \\ Master of Mathematics \\ in \\ Combinatorics and Optimization
}

Waterloo, Ontario, Canada, 2016

(c) Cheolwon Heo 2016 
I hereby declare that I am the sole author of this thesis. This is a true copy of the thesis, including any required final revisions, as accepted by my examiners.

I understand that my thesis may be made electronically available to the public. 


\begin{abstract}
Even-cycle and even-cut matroids are classes of binary matroids that generalize respectively graphic and cographic matroids. We give algorithms to check membership for these classes of matroids. We assume that the matroids are 3-connected and are given by their $(0,1)$-matrix representations. We first give an algorithm to check membership for p-cographic matroids that is a subclass of even-cut matroids. We use this algorithm to construct algorithms for membership problems for even-cycle and even-cut matroids and the running time of these algorithms is polynomial in the size of the matrix representations. However, we will outline only how theoretical results can be used to develop polynomial time algorithms and omit the details of algorithms.
\end{abstract}




\section{Acknowledgements}

I would like to express my gratitude to my advisor Dr. Bertrand Guenin who always supported and encouraged me. Without his assistance and guidance, this thesis would not have come into being. During the program, I had a great time to learn from his immense knowledge and research attitude. 


\section{Table of Contents}

Author's Declaration $\quad$ ii

$\begin{array}{ll}\text { Abstract } & \text { iii }\end{array}$

Acknowledgements $\quad$ iv

List of Figures $\quad$ vii

1 Introduction $\quad 1$

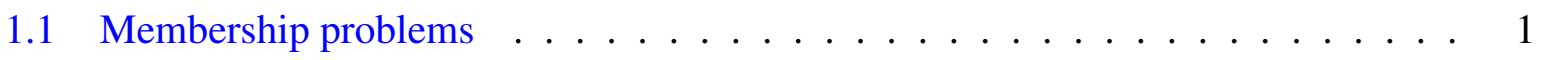

1.2 The case of graphic matroids . . . . . . . . . . . . . . . 3

1.3 What makes the problem difficult? ............... 5

1.3.1 A first bad example . . . . . . . . . . . . . . 5

1.3.2 An even-cycle matroid with an exponential number of inequivalent representations ....................... 7

1.3.3 An even-cut matroid with exponential number of inequivalent representations .......................... 8

1.4 P-graphic and p-cographic matroids . . . . . . . . . . . . . 10

1.5 Thesis Overview . . . . . . . . . . . . . . . . . 12

2 Recognizing even-cycle and even-cut matroids 14

2.1 An algorithm for recognizing even-cycle matroids . . . . . . . . . . . . . . 14 
2.2 An algorithm for recognizing even-cut matroids $\ldots \ldots \ldots \ldots$

2.3 Matroid connectivity . . . . . . . . . . . . . . . . . . 18

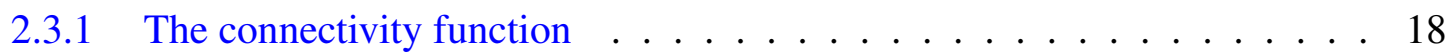

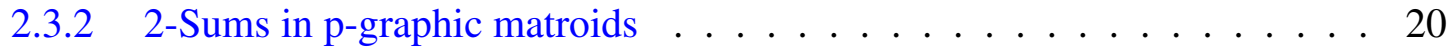

2.4 Bounding the number of representations $\ldots \ldots \ldots$. . . . . . . . . . . . 24

3 Recognizing p-graphic and p-cographic matroids $\quad 27$

3.1 From p-graphic to p-cographic . . . . . . . . . . . . . . . . . 27

3.2 An algorithm for recognizing $\mathrm{p}$-cographic matroids . . . . . . . . . . . . . . 29

3.3 Anemone classes . . . . . . . . . . . . . . . . . . . . . . . . . 30

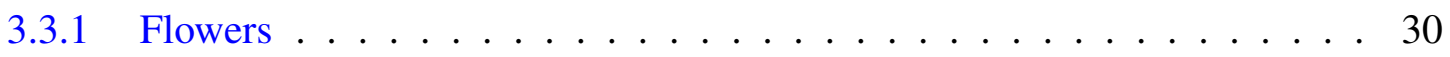

3.3.2 Representations of ordinary anemones . . . . . . . . . . . . . 32

3.3.3 Ordinary anemones related by arranging petals and loose edges . . . . . 40

3.3.4 Bounding the number of representations with anemones . . . . . . . 43

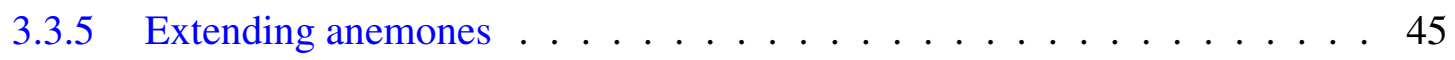

3.4 Bounding the number of representations $\ldots \ldots \ldots \ldots$

3.4.1 Equivalence classes and anemone classes . . . . . . . . . . . . . . . 49

3.4.2 Equivalence classes that are not anemone type $\ldots \ldots \ldots \ldots 1$

3.4.3 Homologous representations . . . . . . . . . . . . . 52

3.4.4 Whitney flip sequences and the gap function . . . . . . . . . . 53

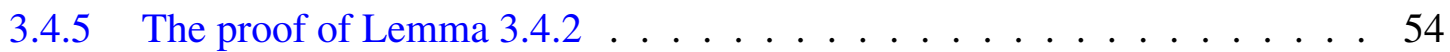

$\begin{array}{ll}\text { References } & 61\end{array}$ 


\section{List of Figures}

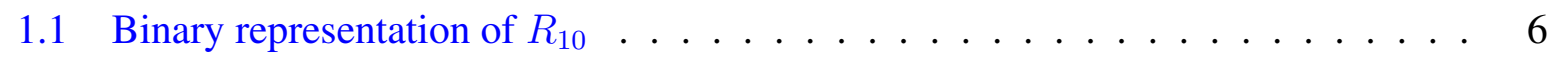

1.2 Inequivalent signed-graph representations of $R_{10} \ldots \ldots \ldots \ldots$

1.3 Inequivalent graft representations of $R_{10} \ldots \ldots \ldots \ldots \ldots$

1.4 Construction of the example in Section $1.3 .2 \ldots \ldots \ldots \ldots$

1.5 Shuffling in the example in Section $1.3 .2 \ldots \ldots \ldots \ldots$

1.6 Construction of the example in Section 1.3.3 . . . . . . . . . . . . . . 9

1.7 Shuffling of the example in Section $1.3 .3 \ldots \ldots$. . . . . . . . . 10

3.1 Folding/unfolding between a $B P$-representation and a $T_{4}$-representation $\ldots \ldots 28$

3.2 Construction of a skewed anemone . . . . . . . . . . . . . . . 33

3.3 Linearly many ordinary anemones $\ldots \ldots \ldots \ldots 4$ 


\section{Chapter 1}

\section{Introduction}

\subsection{Membership problems}

A matroid is graphic if its circuits correspond to the circuits of some graph $G$ (by a cycle in a graph we mean a subset of edges that induces a subgraph where all vertices have even degree, and by a circuit we mean an inclusion-wise minimal cycle, i.e. a subset of edges that induces a connected graph where every vertex has degree two). We say that $G$ is a representation of that matroid and denote the matroid by cycle $(G)$. A matroid is cographic if it is the dual of a graphic matroid, or equivalently, if its circuits correspond to the inclusion-wise minimal cuts of some graph $G$. We say that $G$ is a representation of that matroid and denote the matroid by $\operatorname{cut}(G)$. In [15], Tutte gave a polynomial time algorithm to recognize if a binary matroid (described by its $(0,1)$-matrix representation) is a graphic matroid (and hence to recognize if a binary matroid is a cographic matroid). The main contribution of this thesis is to prove analogous results for two classes of binary matroids arising from graph-like objects, namely, the class of even-cycle matroids and the class of even-cut matroids when the given matroids are 3-connected.

A signed-graph is a pair $(G, \Sigma)$ where $G$ is a graph and $\Sigma$ is a subset of the edges of $G$, i.e. $\Sigma \subseteq E(G)$. We say that a set $B \subseteq E(G)$ is even (resp. odd) if $|B \cap \Sigma|$ is even (resp. odd). We will say that an edge $e$ is odd when $e \in \Sigma$ and even otherwise. A matroid is an even-cycle matroid if its circuits correspond to the inclusion-wise minimal even cycles of some signedgraph $(G, \Sigma)$. We say that $(G, \Sigma)$ is a signed-graph representation (or simply representation) of that matroid and denote the matroid by ecycle $(G, \Sigma)$. Observe that when $\Sigma=\emptyset$, every cycle is even, hence ecycle $(G, \Sigma)=\operatorname{cycle}(G)$. In particular, graphic matroids are even-cycle matroids. Even-cycle matroids are binary matroids, indeed, ecycle $(G, \Sigma)$ can be represented by the $(0,1)$-matrix obtained by appending a row corresponding to the characteristic vector of 
$\Sigma$ to the vertex-edge incidence matrix of $G$. A graft is a pair $(G, T)$ where $G$ is a graph and $T$ is a subset of the vertices of $G$ of even cardinality. Vertices $T$ are called terminals. A cut $\delta_{G}(U):=\{u v \in E(G): u \in U, v \notin U\}^{1}$ is even (resp. odd) if $|U \cap T|$ is even (resp. odd). Note that as we have an even number of terminals, this is well-defined. A matroid is an even-cut matroid if its circuits corresponds to the inclusion-wise minimal even cuts of some graft $(G, T)$. We say that $(G, T)$ is a graft representation (or simply representation) of that matroid and denote the matroid by ecut $(G, T)$. Observe that when $T=\emptyset$, every cut is even, hence ecut $(G, T)=\operatorname{cut}(G)$. In particular, cographic matroids are even-cut matroids. Even-cut matroids are binary matroids, indeed, ecut $(G, T)$ can be represented by the $(0,1)$-matrix obtained by appending a row corresponding to the characteristic vector of a $T$-join (defined in 1.3.1) to the $(0,1)$-matrix representing cut $(G)$. Note, the term representation for an even-cycle (resp. even-cut) matroids will always refer to the signed-graph (resp. graft) representation. We will use the term matrix representation to indicate the $(0,1)$-matrix representing the matroid over the two-element field.

Let $\mathcal{M}$ be a class of binary matroid. By the membership problem for $\mathcal{M}$ we mean the following: we are given a $(0,1)$-matrix representation $A$ of a binary matroid $M$, we return either YES if $M \in \mathcal{M}$ and NO if $M \notin \mathcal{M}$. An algorithm for testing membership problem is polynomial if its running time is polynomial in the size of $A$. The columns of $A$ correspond to the elements $E(M)$ of $M$ and the number of rows of $A$ is given by the rank of $M$ which is bounded by $|E(M)|$. Since $A$ is a $(0,1)$-matrix, saying that the algorithm runs in time polynomial in the size of $A$ is equivalent to saying that the algorithm runs in time polynomial in the number of elements of $M$. The main results in the paper are,

(1) A polynomial time algorithm for testing membership in the class of even-cycle matroids when the given matroids are 3-connected;

(2) A polynomial time algorithm for testing membership in the class of even-cut matroids when the given matroids are 3-connected.

A class $\mathcal{M}$ of matroid is minor closed if for all $M \in \mathcal{M}$ every minor $N$ of $M$ is in $\mathcal{M}$. Evencycle and even-cut matroids are minor closed classes of matroids. A binary matroid $M$ is minimally non-even-cycle (resp. minimally non-even-cut) if it is not an even-cycle (resp. even-cut) matroid but every proper minor of $M$ is an even-cycle (resp. even-cut) matroid. The following theorem is proven by the Matroid Minors Project [8].

Theorem 1.1.1. Every minor-closed class of binary matroids are well-quasi-ordered.

\footnotetext{
${ }^{1}$ We omit the sub-index $G$ when there is no ambiguity.
} 
It follows from Theorem 1.1.1 that minimally non-even-cycle (resp. non-even-cut) matroids have bounded sizes. The algorithm in (1) returns a compact certificate for membership. Namely, if the matroid, say $M$, being tested is an even-cycle matroid, the algorithm returns a signed-graph that is a representation of $M$. The algorithm in (2) returns a compact certificate for membership If the matroid, say $M$, being tested is an even-cut matroid, the algorithm returns a graft that is a representation of $M$.

In this thesis, we do not give all the details of the algorithms, but we focus on the theoretical underpinnings of the algorithms, namely the fact that we can keep track of the representations. We will outline only how these theoretical results can be used to develop polynomial time algorithms. We prove (1) and (2) are polynomial, but we do not work out the exact bound. Furthermore, the polynomial bound will depend on some constant efficient $c$ that arises from the Matroid Minors Project and that has no explicit bound (see [8]). However, the algorithm does not use the value $c$ for its computation.

\subsection{The case of graphic matroids}

Before identifying some of the challenges in designing a polynomial time algorithm for testing membership in even-cycle or even-cut matroid, we will sketch how the membership problem can be solved for the class of graphic matroids. The key property is the following result of Whitney in [17] that under some mild connectivity assumption, graphic matroids have a unique representation,

Theorem 1.2.1. If $M$ is a 3-connected graphic matroid there exists a unique graph $G$ with $\operatorname{cycle}(G)=M .^{2}$

We review the definition of $k$-connected matroids in Section 2.3.1. Given a matroid $M$ and $I, J \subseteq E(M)$ where $I \cap J=\emptyset$, the matroid obtained by deleting elements $I$ and contracting elements $J$ is denoted by $M \backslash I / J$. Note, that the order in which the minor operations are conducted do not matter so the notation is well defined. Similarly, we denote by $G \backslash I / J$ the graph obtained from $G$ by deleting edges $I$ and contracting edges $J$. There is a one-to-one correspondence between minor operations in a graphic matroid and minor operations in its representation, namely,

Remark 1.2.2. Let $I, J \subseteq E(G)$ where $I \cap J=\emptyset$. Then cycle $(G) \backslash I / J=$ cycle $(G \backslash I / J)$.

\footnotetext{
${ }^{2}$ We will consider two graphs that only differ in vertex labels or isolated vertices to be identical.
} 
Note, that the Remark 1.2.2 implies in particular that the class of graphic matroids is closed under minors. If $M^{\prime}$ is obtained from $M$ by contracting (resp. deleting) some element $e$ we say that $M$ is obtained from $M^{\prime}$ by uncontracting (resp. undeleting) element $e$. We use the analogous definition for graphs as well. Suppose $M$ is a graphic representation with representation $G$ and let $M^{\prime}=M \backslash I / J$ be a minor of $M$. Then by the previous remark, $G$ arises from a representation of $M^{\prime}$ by undeleting a set of edges $I$ and uncontracting a set of edges $J$.

We are now ready to sketch an algorithm for testing if a matroid $M$ is graphic. We will limit ourselves to the case where $M$ is 3-connected. In [13] Seymour proved that there exists a sequence of 3-connected matroids $M_{1}, M_{2}, \ldots, M_{k}$ where $M_{1}, M_{2}, \ldots, M_{k}$ are 3-connected, $M_{k}$ is the graphic matroid of a wheel (possibly $K_{4}$ ) or the non-graphic matroid of a whirl and for every $i \in[k-1]^{3}, M_{i+1}$ is isomorphic to a single element contraction or deletion of $M_{i}$. Moreover, this sequence of 3-connected matroids can be constructed in time polynomial in $E(M)$. If $M_{k}$ is non-graphic, then so is $M$. We may assume $M_{k}$ is graphic. Let $G_{k}$ denote the wheel for which cycle $\left(G_{k}\right)=M_{k}$. Suppose that iteratively we constructed representations $G_{i}$ for $i=r, \ldots, k$. If $r=1$ then $M_{1}=M$ is graphic. Otherwise $M_{r-1}$ is obtained from $M_{r}$ by undeleting (or uncontracting) some element $e$. If $M_{r-1}$ is graphic then its representation $G_{r-1}$ is obtained by undeleting (or uncontracting) some edge $e$ from a representation of $M_{r}$. But by Theorem 1.2.1 the representation of $M_{r}$ is unique, it is $G_{r}$. Thus it suffices to check if we can undelete (or uncontract) an edge of $G_{r}$ to get a representation of $M_{r-1}$. We omit a description of this last step but refer the interested reader to [14] and [3] for a detailed explanation of how this can be carried.

We will see that the condition that $M$ be 3-connected cannot be omitted in Theorem 1.2.1. Given a graph $G$ and $X \subseteq E(G)$, we denote by $V_{G}(X)$ the set of vertices spanned by edges $X$, i.e. $V_{G}(X)=V(G[X])$. We denote by $\mathcal{B}_{G}(X)$ the set $V_{G}(X) \cap V_{G}(\bar{X})^{4}$; i.e. $\mathcal{B}_{G}(X)$ is the set of vertices in the boundary of $X$ and $\bar{X}$. Consider a graph $G$ with $X \subseteq E(G)$ and $|X|,|\bar{X}| \geq 2$ where $\mathcal{B}_{G}(X)$ consists of two vertices $u, v$. Let $G^{\prime}$ be obtained by identifying vertex $u$ and $v$ of $G[X]$ with vertices $v$ and $u$ of $G[\bar{X}]$ (in that order). We say that $G^{\prime}$ is obtained from $G$ by a Whitney-flip on $X$. We also call the operation that consists of identifying two components of a graph, or splitting two blocks of a graph a Whitney flip. It can be readily checked that if $G$ and $G^{\prime}$ are related by a sequence of Whitney-flips, then they have the same cycles, i.e. they are representations of the same graphic matroid. In [17], Whitney proved the converse namely,

Theorem 1.2.3. Any two representations of a graphic matroid are related by a sequence of Whitney flips.

\footnotetext{
${ }^{3}[k]=\{1, \ldots, k\}$.

${ }^{4} \bar{S}$ denotes the complement of set $S$.
} 
It is well known that if a graphic matroid $M$ is 3-connected, then every representation $G$ of $M$ is 3-connected. Since there are no Whitney-flip possible in a 3-connected graph, Theorem 1.2.1 follows immediately from Theorem 1.2.3. Theorem 1.2.3 provides a very precise description of the set of all representations of a graphic matroid. We will see that the situation is markedly worse in the case of even-cycle and even-cut matroids.

\subsection{What makes the problem difficult?}

\subsubsection{A first bad example}

Unlike graphic matroids, which have a unique graph representation up to Whitney-flips, evencycle and even-cut matroids may have multiple representations up to Whitney-flips, even though they are 3-connected. Before we see examples, we will introduce a natural ways to attain signedgraph (resp. graft) representations from one signed-graph (resp. graft) representation.

Two graphs $G$ and $G^{\prime}$ are equivalent if $G$ and $G^{\prime}$ are related by a sequence of Whitneyflips. Since cycle $(G)=\operatorname{cycle}\left(G^{\prime}\right)$ it follows in particular, that for any $\Sigma \subseteq E(G)$ we also have $\operatorname{ecycle}(G, \Sigma)=\operatorname{ecycle}\left(G^{\prime}, \Sigma\right)$. The set $\Sigma^{\prime} \subseteq E(G)$ is a signature of $(G, \Sigma)$ if ecycle $(G, \Sigma)=$ ecycle $\left(G, \Sigma^{\prime}\right)$. It can be readily checked that $\Sigma^{\prime}$ is a signature if and only if $\Sigma^{\prime}=\Sigma \triangle \delta(U)$ for some $U \subseteq V(G)$. We say that $\left(G, \Sigma^{\prime}\right)$ is obtained by resigning $(G, \Sigma)$. Thus $(G, \Sigma)$ and $\left(G^{\prime}, \Sigma^{\prime}\right)$ are representations of the same even-cycle matroid if they are related by resigning and Whitney-flips. We will call such a pair of signed-graphs, equivalent. Note that this is indeed an equivalence relation (see [10]), thus we can partition the set of all representations of an evencycle matroid into equivalence classes.

We say that a subset of edges $J$ is a $T$-join of $G$ where $(G, T)$ is a graft, if the vertices of odd degree of $G[J]$ is given by $T$. A graft $(G, T)$ is equivalent to a graft $\left(G^{\prime}, T^{\prime}\right)$ if $G^{\prime}$ is equivalent to $G$ and there exists a $T$-join of $G$ that is a $T^{\prime}$-join of $G^{\prime}$. Note that as $G$ and $G^{\prime}$ have the same cycles this implies readily that for every $J \subseteq E(G), J$ is a $T$-join of $G$ if and only if $J$ is a $T^{\prime}$-join of $G^{\prime}$. Clearly, if $(G, T)$ and $\left(G^{\prime}, T^{\prime}\right)$ are equivalent then ecut $(G, T)=\operatorname{ecut}\left(G^{\prime}, T^{\prime}\right)$ since cocycles of ecut $(G, T)$ are precisely the cycles of $G$ and the $T$-joins of $(G, T)$. Note that this is indeed an equivalence class (see [6]), thus we can partition the set of all representations of an even-cut matroid into equivalence classes.

Now, we will introduce an example with multiple inequivalent representations. The matroid $R_{10}$ is a 4-connected binary matroid with the following matrix representation in Figure 1.1 (see [7]). The matroid $R_{10}$ is an even-cycle matroid and it has six inequivalent representations that are all isomorphic to the signed-graph $\left(K_{5}, E\left(K_{5}\right)\right)$ (see [11]). We introduce two examples 


$$
\left(\begin{array}{llllllllll}
a & b & c & d & e & f & g & h & i & j \\
1 & 0 & 0 & 0 & 0 & 1 & 1 & 0 & 0 & 1 \\
0 & 1 & 0 & 0 & 0 & 1 & 1 & 1 & 0 & 0 \\
0 & 0 & 1 & 0 & 0 & 0 & 1 & 1 & 1 & 0 \\
0 & 0 & 0 & 1 & 0 & 0 & 0 & 1 & 1 & 1 \\
0 & 0 & 0 & 0 & 1 & 1 & 0 & 0 & 1 & 1
\end{array}\right)
$$

Figure 1.1: Binary representation of $R_{10}$.

in Figure 1.2. The matroid $R_{10}$ is also an even-cut matroid and it has ten inequivalent represen-
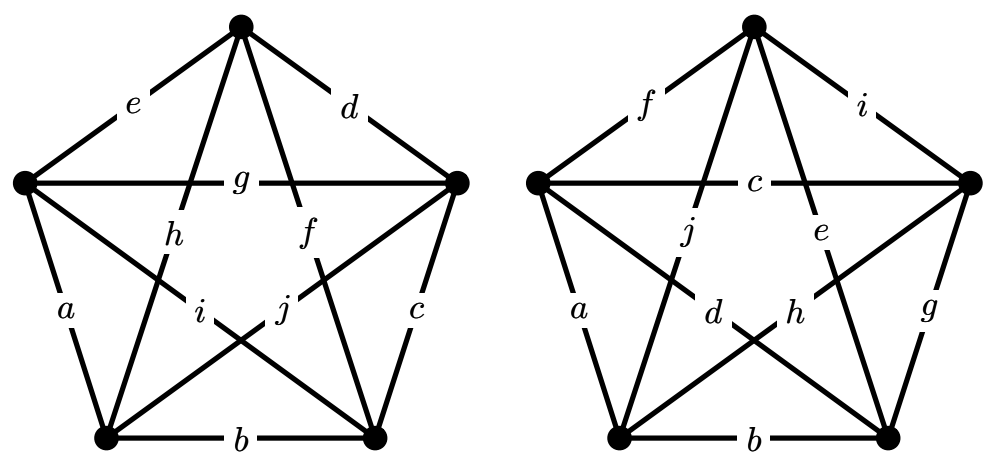

Figure 1.2: Inequivalent signed-graph representations of $R_{10}$. All edges are odd. The edge labelling is the same as in Figure 1.1.

tations that are all isomorphic to the graft given in Figure 1.3. We introduce two examples in Figure 1.3. Figure 1.2 and Figure 1.3 show that it is possible for a 4-connected even-cycle (or even-cut) matroid to have multiple inequivalent representations. Thus unlike the case of graphic matroid, a membership algorithm for either even-cycle of even-cut matroids would need to keep track of multiple representations. This would not be a problem as long as the number of pairwise inequivalent representations is bounded by a polynomial in the number of elements of matroids. Alas we will see that this is not case for the class of even-cycle matroid, nor is it the case for the class of even-cut matroids. 

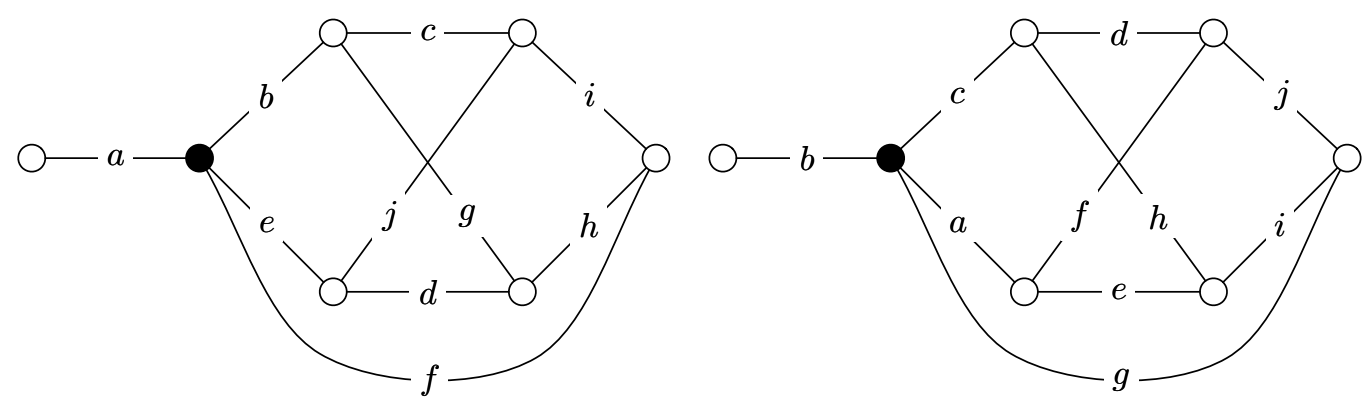

Figure 1.3: Inequivalent graft representations of $R_{10}$. White vertices are terminals. The edge labelling is the same as in Figure 1.1

\subsubsection{An even-cycle matroid with an exponential number of inequivalent representations}

In this section, we will introduce an even-cycle matroid that has an exponential number of pairwise inequivalent representations. A mixed graph is a graph with both edges and directed edges. Let $\vec{H}$ be a mixed graph with exactly two directed edges, say $L$ and $R$, that are disjoint. Construct $G$ from $\vec{H}$ by identifying the tail of $L$ with the tail of $R$; by identifying the head of $L$ with the head of $R$; and by removing $L$ and $R$. Denote by $s_{1}$ (resp. $s_{2}$ ) the tail (resp. head) of $L$ in $\vec{H}$. Let $\Sigma:=\delta_{\vec{H}}\left(s_{1}\right) \triangle \delta_{\vec{H}}\left(s_{2}\right)$. Then $(G, \Sigma)$ is a signed graph. Suppose we have a sets $X_{0}, X_{1}, \ldots, X_{n}$ where $n \geq 2$ with the following properties: (i) $X_{0}=\{L\}, X_{n}=E(\vec{H}) \backslash\{R\}$; (ii) for all $i \in[n], X_{i-1} \subset X_{i}, V_{\vec{H}}\left(X_{i-1}\right) \subset V_{\vec{H}}\left(X_{i}\right)$ and $\left|\mathcal{B}_{\vec{H}}\left(X_{i}\right)\right|=2$. For all $i \in[n]$, let $P_{i}=X_{i}-X_{i-1}$. We illustrate the construction of $(G, \Sigma)$ in Figure 1.4. Note that we can construct an example where $n=\Theta(|E(G)|)$ and ecycle $(G, \Sigma)$ is 3-connected. Now, construct a new mixed graph $\vec{H}^{\prime}$ from $\vec{H}$ by repeatedly doing Whitney-flips on sets $X_{i}$ for all $i \in I \subseteq[n]$ so that the ends of $L$ and $R$ remain disjoint. Let $\left(G^{\prime}, \Sigma^{\prime}\right)$ be the signed-graph that arises from $\vec{H}^{\prime}$ by the same way in the construction of $(G, \Sigma)$. We say that $(G, \Sigma)$ and $\left(G^{\prime}, \Sigma\right)$ are related by shuffling $\left\{P_{i} \mid i \in I\right\}$. We illustrate this shuffling in Figure 1.5. It can be readily checked in [10] that ecycle $(G, \Sigma)=\operatorname{ecycle}\left(G^{\prime}, \Sigma^{\prime}\right)$. It is now straightforward to see that we can have an exponential number (in the number of elements) of inequivalent signed-graph representations all related by shuffling subsets of edges. Hence, we can have an exponential number of pairwise inequivalent representations for an even-cycle matroid. In particular, it implies that a polynomial time recognition algorithm for even-cycle matroids cannot record the set of all pairwise inequivalent representations. 

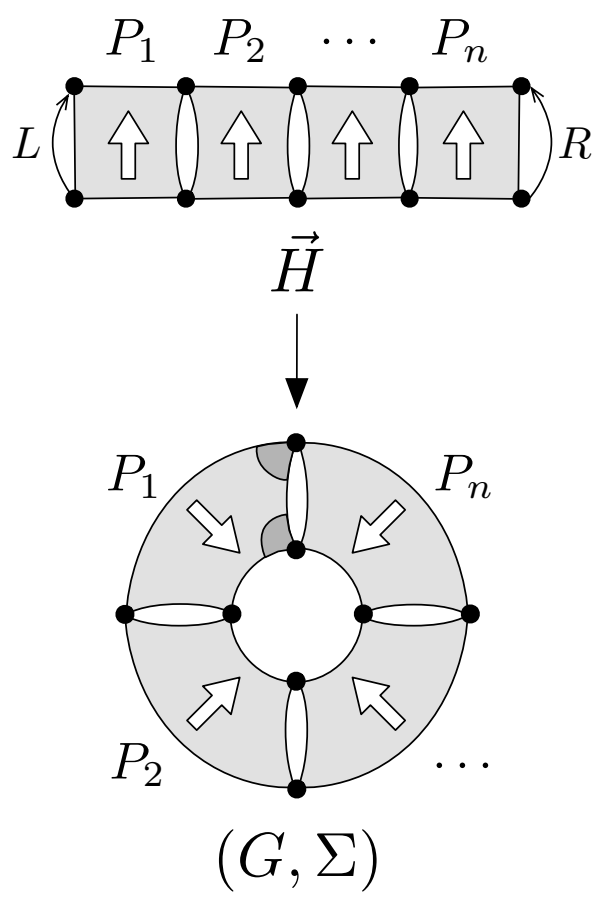

Figure 1.4: Construction of the example in Section 1.3.2. Shaded edges are odd.

\subsubsection{An even-cut matroid with exponential number of inequivalent repre- sentations}

In this section, we will introduce an even-cut matroid that has an exponential number of inequivalent representations. Consider a graft $(G, T)$ where $|T|=4$. Suppose that we have a partition $P_{1}, P_{2}, \ldots, P_{n}$ of $E(G)$ and for every $i \in[n]$, we have $\mathcal{B}_{G}\left(P_{i}\right)=T$. We illustrate the construction of $(G, T)$ in Figure 1.6. Note that we can construct an example where $n=\Theta(|E(G)|)$ and $\operatorname{ecut}(G, T)$ is 3-connected. For every $i \in[n]$ let $G_{i}=G\left[P_{i}\right]$. Denote by $t_{1}, t_{2}, t_{3}, t_{4}$ the terminal vertices $T$. For every $i \in I \subseteq[n]$ let $G_{i}^{\prime}$ be a graph constructed from $G_{i}$ by relabelling the terminals in arbitrary one of three possible ways, (i) interchange the labels of $t_{1}$ and $t_{2}$ the labels of $t_{3}$ and $t_{4}$; (ii) interchange the labels of $t_{1}$ and $t_{3}$ the labels of $t_{2}$ and $t_{4}$; (iii) interchange the labels of $t_{1}$ and $t_{4}$ the labels of $t_{2}$ and $t_{3}$. Now let $G^{\prime}$ be obtained from $G$ by identifying vertices $t_{1}$ (resp. $\left.t_{2}, t_{3}, t_{4}\right)$ in each of $G_{i}$ for $i \in[n]$. Then $\left(G^{\prime}, T\right)$ is a graft. We say that $(G, T)$ and $\left(G^{\prime}, T\right)$ are related by shuffling $\left\{P_{i} \mid i \in I\right\}$. We illustrate this shuffling in Figure 1.7. It can be readily checked in [6] that $\operatorname{ecut}(G, T)=\operatorname{ecut}\left(G^{\prime}, T\right)$. It is now straightforward to see 


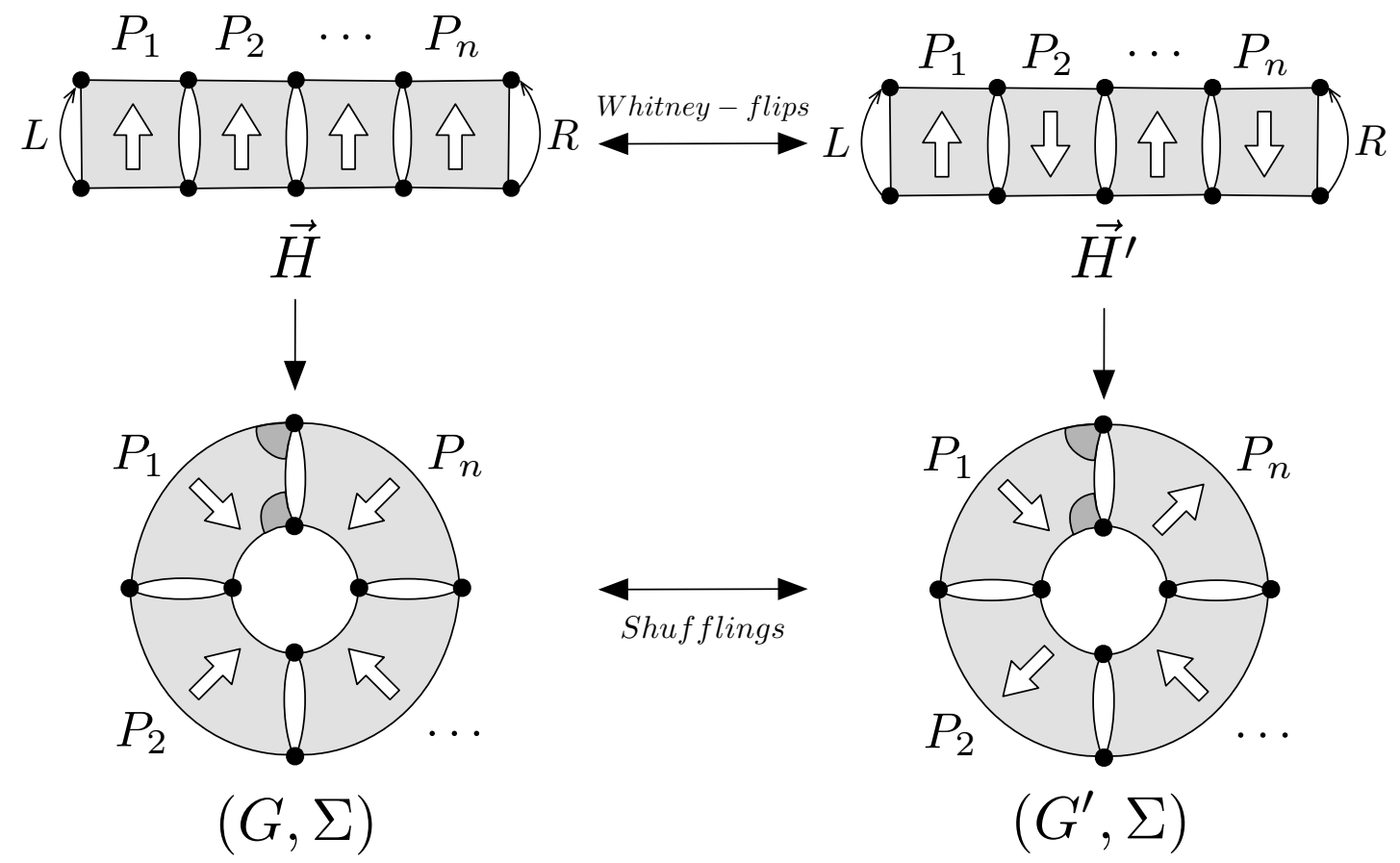

Figure 1.5: Shuffling in the example in Section 1.3.2. Shaded edges are odd.

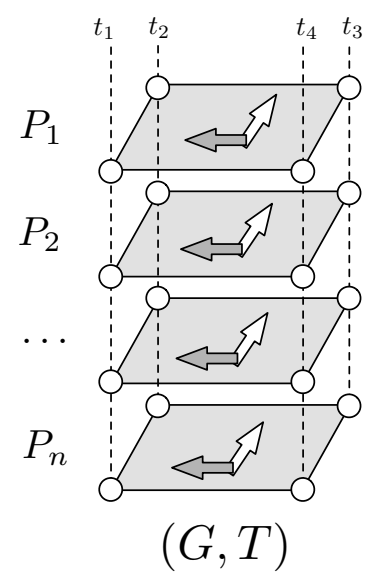

Figure 1.6: Construction of the example in Section 1.3.3. White vertices are terminals. Dotted edges mean identifying vertices. 


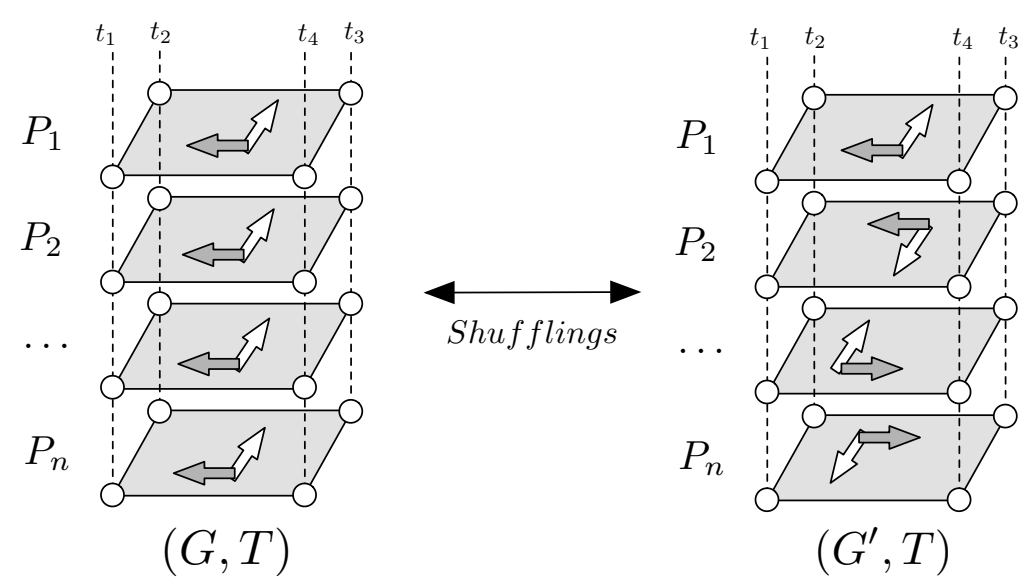

Figure 1.7: Shuffling of the example in Section 1.3.3. White vertices are terminals.

that we can have an exponential number (in the number of elements) of inequivalent graft representations all related by shuffling subsets of edges. Hence, we can have an exponential number of pairwise inequivalent representations for an even-cut matroid. In particular, it implies that a polynomial time recognition algorithm for even-cut matroids cannot record the set of all pairwise inequivalent representations.

\subsection{P-graphic and p-cographic matroids}

In Section 1.3.2 and Section 1.3.3, we showed that there exist examples that have an exponential number of inequivalent representations. In this section, we will introduce a subclass of evencycle matroids, p-graphic matroids, such that even-cycle matroids that are not p-graphic are nicely behaved. We also introduce a subclass of even-cut matroids, $p$-cographic matroids, such that even-cut matroids that are not p-cographic are nicely behaved.

Before we proceed, we require a number of definitions from [11]. Denote by $\operatorname{loop}(H)$ the set of loops of a graph $H$. Consider a graph $H$ and a vertex $v$ and $\alpha \subseteq \delta_{H}(v) \cup \operatorname{loop}(H)$. We say that $G$ is obtained from $H$ by splitting $v$ into $v_{1}, v_{2}$ according to $\alpha$ if $V(G)=V(H)-\{v\} \cup\left\{v_{1}, v_{2}\right\}$ and for every $e=(u, w) \in E(H)$ :

(1) if $e \notin \delta_{H}(v) \cup \operatorname{loop}(H)$, then $e=(u, w) \in E(G)$; 
(2) if $e \in \operatorname{loop}(H)-\alpha$, then $e=(u, w) \in E(G)^{5}$;

(3) if $e \in \operatorname{loop}(H) \cap \alpha$, then $e=\left(v_{1}, v_{2}\right) \in E(G)$;

(4) if $e \in \delta_{H}(v) \cap \alpha$ and $w=v$ then $e=\left(u, v_{1}\right) \in E(G)$;

(5) if $e \in \delta_{H}(v)-\alpha$ and $w=v$ then $e=\left(u, v_{2}\right) \in E(G)$.

Note that if you identify $v_{1}$ and $v_{2}$ in $H$ you get the graph $G$ back. Consider a signed graph $(G, \Sigma)$ we say that $u \in V(G)$ is a blocking vertex if every odd circuit of $(G, \Sigma)$ uses $u$. Equivalently, $u$ is a blocking vertex if there exists a signature $\Gamma$ of $(G, \Sigma)$ where $\Gamma \subseteq \delta_{G}(u) \cup \operatorname{loop}(G)$ (see [11]).

Remark 1.4.1. If an even-cycle matroid has a representation with a blocking vertex then it is graphic.

Proof. Let $(G, \Sigma)$ be the representation. We may assume that $\Sigma \subseteq \delta(u) \cup \operatorname{loop}(G)$ for some vertex $u$. Let $G^{\prime}$ be obtained from $G$ by splitting $u$ according to $\Sigma$. Then clearly cycle $\left(G^{\prime}\right)=$ $\operatorname{ecycle}(G, \Sigma)$.

Consider a signed graph $(G, \Sigma)$ we say that $u, v \in V(G)$ is a blocking pair if every odd circuit of $(G, \Sigma)$ uses at least one of $u, v$. Equivalently, $u, v$ is a blocking pair if there exists a signature $\Gamma$ of $(G, \Sigma)$ where $\Gamma \subseteq \delta_{G}(u) \cup \delta_{G}(v) \cup \operatorname{loop}(G)$ (see [11]). We say that an evencycle matroid $M$ is pinch-graphic (or p-graphic for short) if it has a representation that has a blocking pair. We say that $(G, \Sigma)$ is a blocking pair representation or BP-representation of $M$. The name arises from the fact that if we identify a blocking pair $u, v$ of a representation $(G, \Sigma)$ where $\Sigma \subseteq \delta(u) \cup \delta(v) \cup \operatorname{loop}(G)$ then we obtain a blocking vertex which is, by Remark 1.4.1, a representation of a graphic matroid. In other words, pinch-graphic matroids are one 'pinch' away from being graphic. Observe that examples in Section 1.3.2 have by construction a blocking pair.

Consider a signed graph $(G, \Sigma)$ and $I, J \subseteq E(G)$ where $I \cap J \neq \emptyset$. If $I$ contains an odd circuit, then let $\Gamma=\emptyset$ otherwise there exists a signature $\Gamma$ of $\Sigma$ where $\Gamma \cap I=\emptyset$. Then $(G, \Sigma) / I \backslash J$ denotes the signed graph $(G / I \backslash J, \Gamma)$. Note that minors of signed graph are only defined up to resigning. Analogously to Remark 1.2.2 we have

Remark 1.4.2. Let $I, J \subseteq E(G)$ where $I \cap J=\emptyset$. Then ecycle $(G, \Sigma) / I \backslash J=\operatorname{ecycle}((G, \Sigma) / I \backslash$ $J$ ).

Similarly, for even-cut matroids we have an analogous Remark 1.4.3.

\footnotetext{
${ }^{5}$ If $e$ is a loop on $v$ in $H$, then it can be arbitrarily chosen to be a loop at $v_{1}$ or at $v_{2}$ in $G$.
} 
Remark 1.4.3. If an even-cut matroid has a representation with exactly two terminals then it is cographic.

Proof. Let $(G, T)$ be a representation where $T=\left\{t_{1}, t_{2}\right\}$. Let $G^{\prime}$ be obtained from $G$ by identifying $t_{1}$ and $t_{2}$. Then clearly $\operatorname{cut}\left(G^{\prime}\right)=\operatorname{ecut}(G, T)$.

We say that an even-cut matroid $M$ is pinch-cographic (or p-cographic for short) if it has a representation $(G, T)$ where $|T| \leq 4$. We say that $(G, T)$ is a $T_{4}$-representation of $M$. The name arises from the fact that if we identify say $t_{1}, t_{2} \in T$ in that representation, we obtain a representation with only at most two terminals which is, by Remark 1.4.3, a representation of a cographic matroid. In other words, p-cographic matroids are one 'pinch' away from being cographic. Observe that examples in Section 1.3.3 have by construction four terminals.

Consider a graft $(G, T)$ and $I, J \subseteq E(G)$ where $I \cap J \neq \emptyset$. Let $G^{\prime}=G / I \backslash J$. If $J$ contains an odd cut then $(G, T) / I \backslash J$ denotes $\left(G^{\prime}, \emptyset\right)$. Otherwise then there exists a $T$-join $L$ of $G$ that is disjoint from $J$ and $(G, T) / I \backslash J$ denotes $\left(G^{\prime}, T^{\prime}\right)$ where $T^{\prime}$ is the set of vertices of odd degree of $G^{\prime}[L-I]$. Analogously to Remark 1.4.2 we have

Remark 1.4.4. Let $I, J \subseteq E(G)$ where $I \cap J=\emptyset$. Then ecut $(G, T) / I \backslash J=\operatorname{ecut}((G, T) \backslash I / J)$.

\subsection{Thesis Overview}

In the rest of the thesis, we will introduce two steps for solving the membership problems for even-cycle and even-cut matroids. The main idea starts from the following nice properties in Theorem 1.5.1 and Theorem 1.5.2.

Theorem 1.5.1. There exists a constant c such that every 3-connected even-cycle matroid that is not p-graphic has at most c inequivalent representations.

Theorem 1.5.2. There exists a constant $c$ such that every 3-connected even-cut matroid that is not p-cographic has at most $c$ inequivalent representations.

We postpone the proof of Theorem 1.5.1 and Theorem 1.5.2 until Section 2.4. According to these theorems, to keep track of all representations up to equivalence classes is a good strategy as the number of equivalence classes is bounded by a constant. In Chapter 2, by relying on these nice properties, we assume that we have polynomial time algorithms that recognize p-graphic and pcographic matroids when the given matroids are 3-connected. Then we will solve the weakened membership problems for even-cycle and even-cut matroids using these algorithms, namely, 
(1) Assuming the existence of a polynomial time algorithm recognizing p-graphic matroids, we show how to design a polynomial time algorithm for testing membership for the class of even-cycle matroids when the given matroids are 3-connected;

(2) Assuming the existence of a polynomial-time algorithm recognizing p-cographic matroids, we show how to design a polynomial time algorithm for testing membership for the class of even-cut matroids when the given matroids are 3-connected.

To complete our algorithms, we need algorithms to recognize p-graphic matroids and p-cographic matroids. However, membership problems for $\mathrm{p}$-graphic and p-cographic are equivalent since they are dual to each other.

Proposition 1.5.3. A matroid $M$ is p-graphic if and only if dual of $M$ is p-cographic.

We postpone the proof of Proposition 1.5.3 until Section 3.1. In Chapter 3, we will solve the membership problem for p-cographic matroids, namely,

(3) We show how to design a polynomial time algorithm for testing membership for the class of p-cographic matroids when the given matroids are 3-connected. 


\section{Chapter 2}

\section{Recognizing even-cycle and even-cut matroids}

The goal of this chapter is to solve the membership problems for even-cycle and even-cut matroids when the given matroids are 3-connected. In this chapter, we assume that there exist algorithms for recognizing p-graphic and p-cographic matroids, respectively. These recognition algorithms will be explained in Chapter 3. We also assume that these algorithms return a compact certificate for membership or non-membership. We will use these algorithms to solve membership problem for even-cycle and even-cut matroids. In Section 2.1 and 2.2, we give brief outlines of algorithms for recognizing even-cycle and even-cut matroids. In Section 2.3, we will introduce concepts of matroid connectivity and some ingredients to prove the main results that are used in the algorithms. The proofs for these results will be in Section 2.4.

\subsection{An algorithm for recognizing even-cycle matroids}

To solve the membership problem for even-cycle matroids, we will use a similar strategy for the membership problem for graphic matroids as the one mentioned in Section 1.2. In this section, we give a brief overview of these algorithms as this will also be helpful in outlining the membership algorithm for even-cycle and even-cut matroids. We start with the following observation,

Proposition 2.1.1. Let $\mathcal{M}$ be a minor closed class of binary matroids and suppose that we have a polynomial time algorithm to solve the membership problem for $\mathcal{M}$ when the given matroids are 3-connected. Then given any 3-connected binary matroid $M \notin \mathcal{M}$ described by its (0,1)-matrix 
representation, we can find in polynomial time a 3-connected minor $N$ of $M$ that is minimally not in $\mathcal{M}$.

Proof. Pick $e \in E(M)$. We check if $M / e \notin \mathcal{M}$ and $M / e$ is 3-connected. If it is, we define $M^{\prime}$ as $M / e$. Otherwise we check if $M \backslash e \notin \mathcal{M}$ and $M \backslash e$ is 3-connected. If it is, we define $M^{\prime}$ as $M \backslash e$. We repeat for every element $e \in M$. If $M^{\prime}$ is not defined, then return $N=M$. Otherwise, we repeat the above procedure with $M^{\prime}$. We recursively find minors of $M$, until we find a 3-connected minor $N$ of $M$ that is minimally not in $\mathcal{M}$.

Observe that if a signed graph $(G, \Sigma)$ has a blocking pair, say $u, v$, then for every minor $\left(G^{\prime}, \Sigma^{\prime}\right)$ the vertices $u^{\prime}, v^{\prime}$ corresponding to respectively $u, v$ will form a blocking pair. This proves in particular, that the class of p-graphic matroids is minor closed.

Let $M$ be an even-cycle matroid and let $N=M / I \backslash J$ be a minor of $M$. Suppose that $(G, \Sigma)$ is a representation of $M$. It follows from Remark 1.4.2 that $(H, \Gamma):=(G, \Sigma) / I \backslash J$ is a representation of $N$. We say that representation $(H, \Gamma)$ of $N$ extends to the representation $(G, \Sigma)$ of $M$. Remark 1.4.2 implies that every representation of $M$ is obtained by extending some representation of $N$. It is possible, however, that a single representation of $N$ extends to several inequivalent representations of $M$. If $N$ is a minor of a matroid $M$ then $M$ is a major of $N$. The following results are proved in [10],

Proposition 2.1.2. Let $M$ be a matroid and let $N=M \backslash$ e for some element e. Suppose that $N$ is an even-cycle matroid. Let $\mathcal{F}$ be an equivalence class of representations of $N$. Let $\mathcal{F}^{\prime}$ be the set of all extensions of $\mathcal{F}$ to $M$. Then $\mathcal{F}^{\prime}$ is contained in at most one equivalence class of the representations of $M$.

Proposition 2.1.3. Let $M$ be a matroid without loops and coloops, and let $N=M /$ e for some element e. Suppose that $N$ is an even-cycle matroid that is not graphic. Let $\mathcal{F}$ be an equivalence class of representations of $N$. Let $\mathcal{F}^{\prime}$ be the set of all extensions of $\mathcal{F}$ to $M$. Then $\mathcal{F}^{\prime}$ is contained in at most two equivalence classes of the representations of $M$.

Recall that an equivalence class for an even-cycle matroid is given by the set of all signed graphs that are equivalent to a fixed signed graph that is a representation of that matroid. Partition the set of all signed-graph representations into equivalence classes. Let $\mathcal{E}(M)$ be obtained by selecting one signed-graph from each equivalence classe, i.e. $\mathcal{E}(M)$ is a maximal collection of representations of $M$ that are pairwise inequivalent. The membership algorithm will keep track of $\mathcal{E}(M)$ for even-cycle matroids that are not p-graphic.

Now let us outline how having a polynomial algorithm for membership in the class of pgraphic yields a polynomial algorithm for membership in the class of even-cycle matroids. Note 
that we assume for such an algorithm that if the answer is YES then the algorithm returns a representation that has a blocking pair. Consider a 3-connected binary matroid $M$ given by a $(0,1)$-matrix representation. We check if $M$ is p-graphic; if it is, then it is also an evencycle matroid. Moreover, the membership algorithm returns a representation $(G, \Sigma)$. In fact, $(G, \Sigma)$ has a blocking pair. We can then return $(G, \Sigma)$ as a certificate and stop. Otherwise, because of Proposition 2.1.1 we can find a minor $N$ of $M$ that is minimal with respect to (i) $N$ is non p-graphic and (ii) $N$ is 3-connected. However, unlike the membership problem for graphic matroids in Section 1.2, we cannot use Theorem 1.1.1 to find a small minor of $M$, since 3 -connectivity is not minor-closed. Instead, we have the following similar result.

Theorem 2.1.4. There exists a constant c such that if a binary matroid $N$ is minimal with respect to the following properties,

(1) $N$ is non-p-graphic, and

(2) $N$ is 3-connected,

then $|E(N)|<c$.

We will postpone the proof of Theorem 2.1.4 until Section 2.4. It follows from Theorem 2.1.4 that the size of $N$ is a constant independent of $M$. Then, we check if $N$ an even-cycle matroid (it has constant size so this can be done in constant time). If it is not, then $M$ is not an evencycle matroid either and we can stop. Otherwise, we can use brute force to find $\mathcal{E}(N)$. As $M$ and $N$ are 3-connected, [13] implies that we can construct a sequence of 3-connected matroids $M_{1}, \ldots, M_{k}$ where $M=M_{1}, M_{k}=N$, and for every $i \in[k-1], M_{i+1}$ is obtained from $M_{i}$ by deleting or contracting an element, say $e_{i}$ of $M_{i}$. Inductively, we constructed $\mathcal{E}\left(M_{i+1}\right)$. Using Proposition 2.1.2 and Proposition 2.1.3, we can construct $\mathcal{E}\left(M_{i}\right)$ from $\mathcal{E}\left(M_{i+1}\right)$ in time polynomial in the size of $M_{i}$ and the cardinality of $\mathcal{E}\left(M_{i}\right)$. However, the cardinality of $\mathcal{E}\left(M_{i}\right)$ is bounded by a constant because of Theorem 1.5.1. It follows that $\mathcal{E}\left(M_{i}\right)$ can be constructed in polynomial time in the size of $M_{i}$. If $\mathcal{E}\left(M_{i}\right)=\emptyset$ then $M_{i}$ is not an even-cycle matroid and neither is $M$. Otherwise, if $i=1$ then $M_{i}=M$ is an even-cycle matroid with representations $\mathcal{E}\left(M_{i}\right)$, and if $i>1$ then we recursively construct $\mathcal{E}\left(M_{i-1}\right)$.

\subsection{An algorithm for recognizing even-cut matroids}

We can proceed in a similar way to obtain a polynomial membership algorithm for even-cut matroid using a polynomial recognition algorithm for p-cographic matroids. Observe that if a 
graft $(G, T)$ has at most four terminals then so does every minor $\left(G^{\prime}, T^{\prime}\right)$ of $(G, T)$. This proves in particular, that the class of p-cographic matroids is minor closed.

Let $M$ be an even-cut matroid and let $N=M / I \backslash J$ be a minor of $M$. Suppose that $(G, T)$ is a representation of $M$. It follows from Remark 1.4.4 that $\left(G^{\prime}, T^{\prime}\right):=(G, T) \backslash I / J$ is a representation of $N$. We say that representation $\left(G^{\prime}, T^{\prime}\right)$ of $N$ extends to the representation $(G, T)$ of $M$. Remark 1.4.4 implies that every representation of $M$ is obtained by extending some representation of $N$. It is possible, however, that a single representation of $N$ extends to several inequivalent representations of $M$. Recall that an equivalence class for an even-cut matroid is given by the set of all grafts that are equivalent to a fixed graft that is a representation of that matroid. The following result appears in [6],

Proposition 2.2.1. Let $M$ be a matroid and let $N=M \backslash$ e for some element e. Suppose that $N$ is an even-cut matroid. Let $\mathcal{F}$ be an equivalence class of representations of $N$. Let $\mathcal{F}^{\prime}$ be the set of all extensions of $\mathcal{F}$ to $M$. Then $\mathcal{F}^{\prime}$ is contained in at most one equivalence class of the representations of $M$.

Proposition 2.2.2. Let $M$ be a matroid without loops and coloops, and let $N=M /$ e for some element e. Suppose that $N$ is an even-cut matroid that is not cographic. Let $\mathcal{F}$ be an equivalence class of representations of $N$. Let $\mathcal{F}^{\prime}$ be the set of all extensions of $\mathcal{F}$ to $M$. Then $\mathcal{F}^{\prime}$ is contained in at most two equivalence classes of the representations of $M$.

Let $M$ be an even-cut matroid. Partition the set of all graft representations into equivalence classes. Let $\mathcal{E}(M)$ be obtained by selecting one graft from each equivalence class, i.e. $\mathcal{E}(M)$ is a maximal collection of representations of $M$ that are pairwise inequivalent. The membership algorithm will keep track of $\mathcal{E}(M)$ for even-cut matroids that are not p-cographic.

Now let us outline how having a polynomial algorithm for membership in the class of pcographic yields a polynomial algorithm for membership in the class of even-cut matroids. Note we assume for such an algorithm that if the answer is YES then the algorithm returns a representation that has 4 terminals. Consider a 3-connected binary matroid $M$ given by a $(0,1)$-matrix representation. We check if $M$ is p-cographic; if it is, then it is also an even-cut matroid. Moreover, the membership algorithm returns a representation $(G, T)$. In fact, $|T|=4$. We can then return $(G, T)$ as a certificate and stop. Otherwise, because of Proposition 2.1.1 we can find a minor $N$ of $M$ that is minimal with respect to (i) $N$ is non p-cographic and (ii) $N$ is 3-connected. The following theorem follows the Theorem 2.1.4 since p-cographic matroids are dual of p-graphic matroids by Proposition 1.5.3,

Theorem 2.2.3. There exists a constant $c$ such that if a binary matroid $N$ is minimal with respect to the following properties, 
(1) $N$ is non-p-cographic, and

(2) $N$ is 3-connected,

then $|E(N)|<c$.

It follows from theorem 2.2.3 that the size of $N$ is a constant independent of $M$. Then, we check if $N$ an even-cut matroid (it has constant size so this can be done in constant time). If it is not, then $M$ is not an even-cut matroid either and we can stop. Otherwise, we can use brute force to find $\mathcal{E}(N)$. As $M$ and $N$ are 3-connected, [13] implies that we can construct a sequence of 3-connected matroids $M_{1}, \ldots, M_{k}$ where $M=M_{1}, M_{k}=N$, and for every $i \in[k-1]$, $M_{i+1}$ is obtained from $M_{i}$ by deleting or contracting an element, say $e_{i}$ of $M_{i}$. Inductively, we constructed $\mathcal{E}\left(M_{i+1}\right)$. Using Proposition 2.2.1 and Proposition 2.2.2, we can construct $\mathcal{E}\left(M_{i}\right)$ from $\mathcal{E}\left(M_{i+1}\right)$ in time polynomial in the size of $M_{i}$ and the cardinality of $\mathcal{E}\left(M_{i}\right)$. However, the cardinality of $\mathcal{E}\left(M_{i}\right)$ is bounded by a constant because of Theorem 1.5.2. It follows that $\mathcal{E}\left(M_{i}\right)$ can be constructed in polynomial time in the size of $M_{i}$. If $\mathcal{E}\left(M_{i}\right)=\emptyset$ then $M_{i}$ is not an evencut matroid and neither is $M$. Otherwise, if $i=1$ then $M_{i}=M$ is an even-cut matroid with representations $\mathcal{E}\left(M_{i}\right)$, and if $i>1$ then we recursively construct $\mathcal{E}\left(M_{i-1}\right)$.

\subsection{Matroid connectivity}

\subsubsection{The connectivity function}

Tutte [16] introduced connectivity and separations in matroids. In this section, we review these definitions and present their applications to even-cycle and even-cut matroids. Let $M$ be a matroid with a rank function $r$. For $X \subseteq E(M)$, the connectivity function is defined as $\lambda_{M}(X)=r(X)+r(\bar{X})-r(M)$. A partition $(X, \bar{X})$ of $E(M)$ is $k$-separating if $\lambda_{M}(X) \leq k-1$. It is exactly $k$-separating when equality holds. A partition $(X, \bar{X})$ is a $k$-separation if it is exactly $k$-separating and $|X|,|\bar{X}| \geq k . M$ is $k$-connected if it has no $r$-separations for any $r<k$. We simply say that $M$ is connected if $M$ is 2-connected. Let $G$ be a connected graph and let $X \subseteq E(G)$. The partition $(X, \bar{X})$ is a $k$-separation of $G$ if $|X|,|\bar{X}| \geq k,\left|\mathcal{B}_{G}(X)\right|=k$ and both $G[X]$ and $G[\bar{X}]$ are connected. Note that with this definition two parallel edges of $G$ form a 2 -separation of $G$. A connected graph $G$ is $k$-connected if it has no $r$-separations for any $r<k$.

A signed-graph is bipartite if all its cycles are even. The number of components of a graph $G$ is denoted by $\kappa(G)$. The next propositions from [10] describes the connectivity function for even-cycle matroids. 
Proposition 2.3.1. Let $(G, \Sigma)$ be a non-bipartite signed-graph where $G$ is connected and let $\left(X_{1}, X_{2}\right)$ be a partition of $E(G)$. For $i=1,2$ let $p_{i}=0$ if $\left(G\left[X_{i}\right], \Sigma \cap X_{i}\right)$ is bipartite and let $p_{i}=1$ otherwise. Then

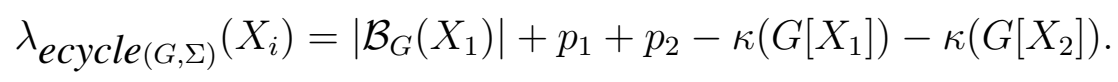

We will use the following application of this proposition,

Proposition 2.3.2. Suppose that ecycle $(G, \Sigma)$ is 3-connected. Let $S$ denote the set of all odd loops. Then

(1) $(G, \Sigma)$ has no even loop,

(2) $|S| \leq 1$ and

(3) $G \backslash S$ is 2-connected.

Moreover, if $G$ has a 2-separation $(X, \bar{X})$, then $(G[X], \Sigma \cap X)$ and $(G[\bar{X}], \Sigma \cap \bar{X})$ are both non-bipartite.

The next propositions [6] describes the connectivity function for even-cut matroids.

Proposition 2.3.3. Let $(G, T)$ be a graft where $T \neq \emptyset$ and $G$ is connected and let $X_{1}, X_{2}$ be a partition of $E(G)$. For $i=1,2$ let $p_{i}=0$ if $(G, T) / \bar{X}_{i}$ has no odd cut and let $p_{i}=1$ otherwise. Then

$$
\lambda_{\text {ecut }(G, T)}\left(X_{i}\right)=\left|\mathcal{B}_{G}\left(X_{1}\right)\right|+p_{1}+p_{2}-\kappa\left(G\left[X_{1}\right]\right)-\kappa\left(G\left[X_{2}\right]\right) .
$$

Given a separation $X$ of $G$, we define the interior of $X$ in $G$ to be $\mathcal{I}_{G}(X)=V_{G}(X)-\mathcal{B}_{G}(X)$. We will use the following application of this proposition,

Proposition 2.3.4. Suppose that ecut $(G, T)$ is 3-connected. Let $S$ denote the set of all odd bridges. Then

(1) $(G, T)$ has no even bridge,

(2) $|S| \leq 1$ and

(3) G/S is 2-connected.

Moreover, if $G$ has a 2-separation $(X, \bar{X})$ then $T \cap \mathcal{I}_{G / \bar{X}}(X)$ and $T \cap \mathcal{I}_{G / X}(\bar{X})$ are both nonempty. 


\subsubsection{2-Sums in p-graphic matroids}

Let $M_{1}$ and $M_{2}$ be binary matroids that have exactly one element, say $e$, in common. The 2-sum $M=M_{1} \oplus_{2} M_{2}$ of $M_{1}$ and $M_{2}$ is defined as the matroid with elements $E\left(M_{1}\right) \triangle E\left(M_{2}\right)$ and circuits that are either circuits of $M_{i}$ avoiding $e$ for $i \in\{1,2\}$ or; are of the form $C_{1} \triangle C_{2}$ where for $i \in\{1,2\}, C_{i}$ is a circuit of $M_{i}$ using $e$. We will use the following results from [9].

Proposition 2.3.5. Let $M$ be a binary matroid and let $X \subseteq E(M)$. Then $(X, \bar{X})$ is a 2separation of $M$ if and only if there exists $M_{1}, M_{2}$ with $E\left(M_{1}\right)-E\left(M_{2}\right)=X$ and $M=$ $M_{1} \oplus_{2} M_{2}$. Moreover, if $M$ is 2-connected then $M_{1}$ and $M_{2}$ are minors of $M$.

Next we present two natural ways of constructing 2-sums of even-cycle matroids in terms of representations. Let $\left(G_{1}, \Sigma\right)$ be a signed graph and let $G_{2}$ be a graph. Suppose that $E\left(G_{1}\right) \cap$ $E\left(G_{2}\right)=\{e\}$ where $e$ is not a loop of $G_{1}$ or $G_{2}$ and suppose also that $e \notin \Sigma$. Let $G$ be obtained from $G_{1}$ and $G_{2}$ by identifying $e$ and then deleting $e$ (we can get two possible graphs in that way and these graphs are related by a single Whitney-flip). Then it can be readily checked that,

$$
\operatorname{ecycle}(G, \Sigma)=\operatorname{ecycle}\left(G_{1}, \Sigma\right) \oplus_{2} \operatorname{cycle}\left(G_{2}\right) .
$$

We then say that $(G, \Sigma)$ is obtained from $\left(G_{1}, \Sigma\right)$ and $G_{2}$ by summing on an edge.

Let $\left(G_{1}, \Sigma_{1}\right)$ and $\left(G_{2}, \Sigma_{2}\right)$ be non-bipartite signed graphs. Suppose that $E\left(G_{1}\right) \cap E\left(G_{2}\right)=$ $\{e\}$ where $e$ is an odd loop of both $\left(G_{1}, \Sigma_{1}\right)$ and $\left(G_{2}, \Sigma_{2}\right)$. Let $G$ be obtained by taking the disjoint union of $G_{1}$ and $G_{2}$ and deleting the loop $e$. Let $\Sigma=\Sigma_{1} \triangle \Sigma_{2}$. Then it can be readily checked that,

$$
\operatorname{ecycle}(G, \Sigma)=\operatorname{ecycle}\left(G_{1}, \Sigma_{1}\right) \oplus_{2} \operatorname{ecycle}\left(G_{2}, \Sigma_{2}\right) .
$$

We then say that $(G, \Sigma)$ is obtained from $\left(G_{1}, \Sigma_{1}\right)$ and $\left(G_{2}, \Sigma_{2}\right)$ by summing on a loop.

By Proposition 2.3.5, to understand 2-sums in p-graphic matroids, we need to understand 2 -separations in p-graphic matroids. Before we proceed, we require a definition. Let $G$ be a graph and let $\left(E_{1}, E_{2}\right)$ be a partition of $E(G)$. Then we define auxiliary graph $\tilde{G}$ of $G$ according to $E_{1}, E_{2}$ as follows:

(1) each component of $G\left[E_{1}\right], G\left[E_{2}\right]$ is a vertex of $\tilde{G}$.

(2) each vertex of $G$ that is incident to two components of $G\left[E_{1}\right], G\left[E_{2}\right]$ is an edge between corresponding vertices in $\tilde{G}$.

Note that every auxiliary graphs are bipartite. 
Proposition 2.3.6. Let $M$ be an even-cycle matroid that is not graphic where $\left(X_{1}, X_{2}\right)$ is a 2separation of $M$. Let $\left(G^{\prime}, \Sigma\right)$ be a representation of $M$. Then there exists a graph $G$ equivalent to $G^{\prime}$ and its auxiliary graph $\tilde{G}$ according to $\left(X_{1}, X_{2}\right)$ where for $i \in\{1,2\}, G_{i}=G\left[X_{i}\right]$ and $\Sigma_{i}=\Sigma \cap X_{i}$, such that either

(1) both $\left(G_{1}, \Sigma_{1}\right)$ and $\left(G_{2}, \Sigma_{2}\right)$ are non-bipartite and $\tilde{G}$ is a 1-path graph,

(2) exactly one of $\left(G_{1}, \Sigma_{1}\right),\left(G_{2}, \Sigma_{2}\right)$ is non-bipartite and $\tilde{G}$ is a 2-circuit graph,

(3) both $\left(G_{1}, \Sigma_{1}\right)$ and $\left(G_{2}, \Sigma_{2}\right)$ are bipartite and $\tilde{G}$ is a union of two 2-circuit graphs sharing one vertex in common.

Moreover, in (3), let $G_{1}$ be the corresponding graph to a vertex of degree 4 in the auxiliary graph and let $G_{2}$ be the union of two components $C_{1}$ and $C_{2}$ respectively corresponding to two vertices of degree 2 in the auxiliary graph. Let $\mathcal{B}_{G}\left(E\left(C_{1}\right)\right)=\left\{a_{1}, a_{2}\right\}$ and let $\mathcal{B}_{G}\left(E\left(C_{2}\right)\right)=\left\{b_{1}, b_{2}\right\}$. Then $\left(\delta\left(a_{1}\right) \cup \delta\left(b_{1}\right)\right) \cap X_{1}$ is a signature of $\Sigma$.

Proof. Let $G$ be a graph equivalent to $G^{\prime}$ such that $\tilde{G}$ has the minimum number of vertices. Since $G$ is connected, $\tilde{G}$ is connected. Let $\left(A_{1}, A_{2}\right)$ be a bipartition of $\tilde{G}$ where for $i \in\{1,2\}, A_{i}$ are components of $G_{i}$.

Claim 1. There exists a cut-edge in $\tilde{G}$ if and only if (1) occurs.

Proof. Suppose that there exists a cut-edge $e=u v$ of $\tilde{G}$ where $u \in A_{1}$ and $v \in A_{2}$. Then deleting $e$ divides $\tilde{G}$ into two components $C_{1}$ containing $u$ and $C_{2}$ containing $v$. By the way of contradiction, let us assume $|V(\tilde{G})| \geq 3$. We may assume that $C_{1}$ contains at least two vertices. Then there exists a vertex $w \in A_{2}$ in $C_{1}$. Let $H$ be a graph obtained from $G$ by splitting the vertex corresponding to $e$ according to $u$ and $v$, and identifying one vertex of the component corresponding to $w$ and one vertex of the component corresponding to $v$. Then $H$ is a graph equivalent to $G$ where $\tilde{H}$ has less number of vertices than $\tilde{G}$, giving a contradiction. Thus, $u, v$ are the only vertices and $\tilde{G}$ is a 1-path graph. Since $\lambda_{M}\left(E\left(M_{1}\right)\right)=1$, by Proposition 2.3.1, both $\left(G_{1}, \Sigma_{1}\right),\left(G_{2}, \Sigma_{2}\right)$ are non-bipartite. The opposite direction is trivial.

Claim 2. There is no path $\left(v_{1}, v_{2}, v_{3}, v_{4}\right)$ of length 3 in $\tilde{G}$ such that $\operatorname{deg}\left(v_{2}\right)=\operatorname{deg}\left(v_{3}\right)=2$. Also, there is no 3-circuit $\left(v_{1}, v_{2}, v_{3}\right)$ in $\tilde{G}$ such that $\operatorname{deg}\left(v_{2}\right)=\operatorname{deg}\left(v_{3}\right)=2$.

Proof. Suppose that there exists a path $\left(v_{1}, v_{2}, v_{3}, v_{4}\right)$ in $\tilde{G}$. Let $X=E\left(v_{2}\right) \cup E\left(v_{3}\right)$ in $G$. Since $(X, \bar{X})$ is a 2-separation of $G$, we can perform a Whitney-flip on $X$ to obtain an auxiliary graph with less number of vertices, giving a contradiction. We can use the same argument for the second result. 
Let $k=\left|\mathcal{B}_{G}\left(E\left(G_{1}\right)\right)\right|$. For $i \in\{1,2\}$, let $p_{i}=0$ if $\left(G_{i}, \Sigma_{i}\right)$ is bipartite and let $p_{i}=1$ otherwise. By Proposition 2.3.1, $k=\kappa\left(G_{1}\right)+\kappa\left(G_{2}\right)+1-p_{1}-p_{2}$. Thus, $|E(\tilde{G})|=k=|V(\tilde{G})|+1-p_{1}-p_{2}$. There are the following cases.

Case 1. Both $\left(G_{1}, \Sigma_{1}\right)$ and $\left(G_{2}, \Sigma_{2}\right)$ are non-bipartite.

Since $p_{1}=p_{2}=1$, we have $|E(\tilde{G})|=|V(\tilde{G})|-1$. Thus, $\tilde{G}$ is a tree. By Claim 1, (1) occurs.

Case 2. Exactly one of $\left(G_{1}, \Sigma_{1}\right)$ and $\left(G_{2}, \Sigma_{2}\right)$ is bipartite.

We may assume $\left(G_{1}, \Sigma_{1}\right)$ is bipartite. Since $p_{1}=0$ and $p_{2}=1,|E(\tilde{G})|=|V(\tilde{G})|$. By Claim 1, there is no vertex of degree 1. Thus, $\tilde{G}$ is a circuit. By Claim 2, $\tilde{G}$ is a 2 -circuit and (2) occurs.

Case 3. Both $\left(G_{1}, \Sigma_{1}\right)$ and $\left(G_{2}, \Sigma_{2}\right)$ are bipartite.

Since $p_{1}=p_{2}=0,|E(\tilde{G})|=|V(\tilde{G})|+1$. By Claim 1, there is no vertex of degree 1 . Thus, in $\tilde{G}$, either every vertex has degree 2 except two vertices of degree 3 , or every vertex has degree 2 except one vertex of degree 4.

Claim 3. Every vertex has degree 2 except one vertex of degree 4 in $\tilde{G}$.

Proof. Suppose that every vertex has degree 2 except two vertices of degree 3 in $\tilde{G}$. Let $u, v$ be vertices of degree 3. Then, there exists a path $P_{1}$ between $u$ and $v$, and consider $H=\tilde{G} \backslash P_{1}$. Since every vertex in $H$ except isolated vertices has degree $2, H$ is a circuit or a union of two disjoint circuits. If $H$ is a union of two disjoint cycles, then each edge of $P_{1}$ is a cut-edge contradicting Claim 1. Thus, $H$ is a cycle containing both $u, v$, so $\tilde{G}$ is a union of three internally disjoint $u, v$-paths $P_{1}, P_{2}, P_{3}$. By Claim 2, length of $P_{1}, P_{2}, P_{3}$ is at most 2. Suppose that $u, v$ are contained in the different partition of $\left(A_{1}, A_{2}\right)$. Then, $P_{1}, P_{2}, P_{3}$ are 1-paths and for $i \in\{1,2\}$, $G_{i}$ is connected. Let $\mathcal{B}_{G}\left(X_{1}\right)=\left\{v_{1}, v_{2}, v_{3}\right\}$. Since $\left(G_{2}, \Sigma_{2}\right)$ is bipartite, we may assume $\Sigma=\Sigma_{1}$. Since $\left(G_{1}, \Sigma_{1}\right)$ is bipartite, $\Sigma_{1}$ is a cut of $G_{1}$. If this cut does not separate $v_{1}, v_{2}, v_{3}$ in $G_{1}$, then $(G, \Sigma)$ is bipartite, giving a contradiction. If this cut separate $v_{1}, v_{2}, v_{3}$ (say, $v_{1}$ and $v_{2}, v_{3}$ ), then $v_{1}$ is a blocking vertex, giving a contradiction by Remark 1.4.1. Thus, $u, v$ are contained in the same partition of $\left(A_{1}, A_{2}\right)$. Thus, $P_{1}, P_{2}, P_{3}$ are 2-paths. We can use the similar argument to show that $(G, \Sigma)$ is graphic, giving a contradiction.

By Claim 3, every vertex has degree 2 except one vertex $u$ of degree 4 in $\tilde{G}$. $\tilde{G} \backslash u$ is a union of disjoint two paths. Thus, $\tilde{G}$ is a union of two circuits sharing one vertex in common. By Claim 2, these circuits are 2-circuits and (3) occurs. We can use the similar argument in Claim 3 to prove the last part.

Now we can prove the following Proposition 2.3.7 and Proposition 2.3.8 about a 2-sum in p-graphic matroids. 
Proposition 2.3.7. Let $M$ be a p-graphic matroid that is not graphic. Suppose there exist matroids $M_{1}$ and $M_{2}$ such that $E\left(M_{1}\right) \cap E\left(M_{2}\right)=\{e\}$ and $M=M_{1} \oplus_{2} M_{2}$. Then for some $i \in\{1,2\}, M_{i}$ is p-graphic and non-graphic but $M_{3-i}$ is graphic.

Proof. Proposition 2.3.5 implies that $M_{1}, M_{2}$ are minor of $M$. It follows that $M_{1}, M_{2}$ are both p-graphic. Moreover, if $M_{1}, M_{2}$ are both graphic then so is $M$, a contradiction. It suffices to show that for some $i \in\{1,2\}, M_{i}$ is graphic. Let $\left(G^{\prime}, \Sigma\right)$ be a BP-representation of $M$ and for $i \in\{1,2\}$, let $X_{i}=E\left(M_{i}\right)-\{e\}$. Apply Proposition 2.3.6 to $M$ and $\left(G^{\prime}, \Sigma\right)$ with the 2-separation $\left(X_{1}, X_{2}\right)$ and let $G$ be the result graph. For $i \in\{1,2\}$, let $G_{i}=G\left[X_{i}\right]$ and let $\Sigma_{i}=\Sigma \cap X_{i}$

Case 1. The outcome (1) occurs.

Then, $(G, \Sigma)$ is obtained by summing on a loop from $\left(G_{1}, \Sigma_{1}\right)$ and $\left(G_{2}, \Sigma_{2}\right)$ where for $i \in\{1,2\}$, $M_{i}=\operatorname{ecycle}\left(G_{i}, \Sigma_{i}\right)$. Suppose that none of $\left(G_{1}, \Sigma_{1}\right)$ and $\left(G_{2}, \Sigma_{2}\right)$ is graphic. By Remark 1.4.1, for $i \in\{1,2\},\left(G_{i}, \Sigma_{i}\right)$ has at least two vertices to intersect all odd circuits. Thus, $(G, \Sigma)$ require at least three vertices to intersect all odd circuits $\left(\right.$ as $\left(G_{1}, \Sigma_{1}\right)$ and $\left(G_{2}, \Sigma_{2}\right)$ share at most one vertex). But this contradicts the fact that $(G, \Sigma)$ is a BP-representation.

Case 2. The outcome (2) occurs.

Then, $(G, \Sigma)$ is obtained by summing on an edge from $\left(G_{1}, \Sigma_{1}\right)$ and $\left(G_{2}, \Sigma_{2}\right)$ where for $i \in$ $\{1,2\}, M_{i}=\operatorname{ecycle}\left(G_{i}, \Sigma_{i}\right)$. Suppose that $\left(G_{1}, \Sigma_{1}\right)$ is bipartite. Then, $M_{1}$ is graphic.

Case 3. The outcome (3) occurs.

Let $G_{1}$ be the corresponding graph to a vertex of degree 4 in the auxiliary graph. Let $G_{2}$ be the union of two components $C_{1}$ and $C_{2}$ respectively corresponding to two vertices of degree 2 in the auxiliary graph. Let $\mathcal{B}_{G}\left(E\left(C_{1}\right)\right)=\left\{a_{1}, a_{2}\right\}$ and let $\mathcal{B}_{G}\left(E\left(C_{2}\right)\right)=\left\{b_{1}, b_{2}\right\}$. Since $\left(\delta\left(a_{1}\right) \cup \delta\left(b_{1}\right)\right) \cap X_{1}$ is a signature of $\Sigma$, an edge set $C \subseteq E\left(M_{2}\right)$ is a circuit of $M_{2}$ if and only if $C$ is either a circuit of $G_{2}$ or the union of $\{e\}$ and an inclusion-wise minimal $\left\{a_{1}, a_{2}, b_{1}, b_{2}\right\}$-join of $G_{2}$. Now construct a graph $H$ from $G_{2}$ by (i) identifying $a_{1}$ and $b_{1}$, and (ii) adding an edge $e=\left(a_{2}, b_{2}\right)$. Then, cycle $(H)=M_{2}$. Thus, $M_{2}$ is graphic.

Proposition 2.3.8. Let $M_{1}$ be a p-graphic matroid and let $M_{2}$ be a graphic matroid. Suppose that $E\left(M_{1}\right) \cap E\left(M_{2}\right)=\{e\}$. Then $M=M_{1} \oplus_{2} M_{2}$ is p-graphic. Moreover, if $M_{1}$ is not graphic, then so is $M$.

Before we can proceed to the proof of Proposition 2.3.8, we require some preliminaries. Consider a signed graph $(G, \Sigma)$ and vertices $v_{1}, v_{2} \in V(G)$ where $\Sigma \subseteq \delta_{G}\left(v_{1}\right) \cup \delta_{G}\left(v_{2}\right) \cup \operatorname{loop}(G)$. We can construct a signed graph $\left(G^{\prime}, \Sigma\right)$ from $(G, \Sigma)$ by replacing the incidences of every odd edge $e$ as follows: 
- if $e=v_{1} v_{2}$ in $G$, then $e$ becomes a loop in $G^{\prime}$ incident to $v_{1}$;

- if $e$ is a loop in $G$, then $e=v_{1} v_{2}$ in $G^{\prime}$;

- if $e=x v_{i}$, for $i \in\{1,2\}$ and $x \neq v_{1}, v_{2}$, then $e=x v_{3-i}$ in $G^{\prime}$.

In this case, we say that $\left(G^{\prime}, \Sigma\right)$ is obtained from $(G, \Sigma)$ by a Lovász-flip. In [11] it is shown that ecycle $\left(G^{\prime}, \Sigma\right)=\operatorname{ecycle}(G, \Sigma)$ i.e. they are both representations of the same p-graphic matroid. An immediate corollary is the following observation,

Remark 2.3.9. Let $M$ be a connected p-graphic matroid and let e be some element of $M$. Then there exists a BP-representation of $M$ where e is not a loop.

Proof of Proposition 2.3.8. Let $e$ be the unique element in $E\left(M_{1}\right) \cap E\left(M_{2}\right)$. Let $\left(G_{1}, \Sigma\right)$ be a BP-representation of $M_{1}$. Because of Remark 2.3.9 we may assume that $e$ is not an odd loop of $\left(G_{1}, \Sigma_{1}\right)$. By resigning we may also assume that $e \notin \Sigma_{1}$. Let $G_{2}$ be representation of $M_{2}$. Then by summing $\left(G_{1}, \Sigma\right)$ and $G_{2}$ on edge $e$ we obtain a BP-representation $(G, \Sigma)$ of $M$. The last part directly follows from Proposition 2.3.5.

\subsection{Bounding the number of representations}

The following results respectively appears in [10] and [6].

Theorem 2.4.1. Let $N$ be a 3-connected even-cycle matroid that is not p-graphic. Let $M$ be a 3 -connected major of $N$. For every equivalence class $\mathcal{F}$ of $N$, the set of extensions of $\mathcal{F}$ to $M$ is the union of at most two equivalence classes.

Theorem 2.4.2. Let $N$ be a 3-connected even-cut matroid that is not p-cographic. Let $M$ be a 3 -connected major of $N$. For every equivalence class $\mathcal{F}$ of $N$, the set of extensions of $\mathcal{F}$ to $M$ is the union of at most two equivalence classes.

Now, we are ready to prove Theorem 1.5.1 and Theorem 1.5.2.

Proof of Theorem 1.5.1. Let $c$ be the constant from Theorem 2.1.4. Let $M$ be a 3-connected even-cycle matroid that is not p-graphic. Let $N$ be a minimal matroid with respect to (1) $N$ is non-p-graphic and (2) $N$ is 3-connected. By the Theorem 2.1.4, $|E(N)|<c$. Let $c^{\prime}$ be the maximum number of inequivalent representations for a matroid on at most $c$ elements. Then $|\mathcal{S}(N)| \leq c^{\prime}$. It follows from Theorem 2.4.1 that $|\mathcal{S}(M)| \leq 2 c^{\prime}$. 
Proof of Theorem 1.5.2. Let $c$ be the constant from Theorem 2.2.3. Let $M$ be a 3-connected even-cut matroid that is not p-cographic. Let $N$ be a minimal matroid with respect to (1) $N$ is non-p-cographic and (2) $N$ is 3-connected. By the Theorem 2.2.3, $|E(N)|<c$. Let $c^{\prime}$ be the maximum number of inequivalent representations for a matroid on at most $c$ elements. Then $|\mathcal{S}(N)| \leq c^{\prime}$. It follows from Theorem 2.4.2 that $|\mathcal{S}(M)| \leq 2 c^{\prime}$.

Now it remains that to prove Theorem 2.1.4. Before we prove Theorem 2.1.4, we need following lemmas.

Lemma 2.4.3. Let $N$ be a binary matroid that is minimally non-p-graphic. Then $N$ is connected. Moreover, if $N$ is not 3-connected, then $N$ has at most one 2-separation.

Proof. Suppose $N$ is not connected. Then there exists a 1-separation $\left(X_{1}, X_{2}\right)$ of $N$. Since $N$ is a minimally non-p-graphic matroid, $\left.N\right|_{X_{1}}$ and $\left.N\right|_{X_{2}}$ are p-graphic. For $i \in\{1,2\}$, let $\left(G_{i}, \Sigma_{i}\right)$ be BP-representations for $\left.N\right|_{X_{i}}$. By Remark 2.3.1, since $\left(X_{1}, X_{2}\right)$ is a 1-separation of $N$, at least one of $\left(G_{i}, \Sigma_{i}\right)$ is bipartite. Thus, $N$ is p-graphic, giving a contradiction.

For the second part, we assume that $N$ has at least two 2-separations. By [1], there exists $N_{1}, N_{2}, N_{3}$ such that $N=N_{1} \oplus_{2} N_{2} \oplus_{2} N_{3}$ where $E\left(N_{1}\right) \cap E\left(N_{2}\right)=\{e\}, E\left(N_{2}\right) \cap E\left(N_{3}\right)=\{f\}$ and $E\left(N_{1}\right) \cap E\left(N_{3}\right)=\emptyset$. Since $N$ is minimally non-p-graphic, $N_{1} \oplus_{2} N_{2}$ and $N_{2} \oplus_{2} N_{3}$ are p-graphic. By Proposition 2.3.8, $N_{1}$ and $N_{3}$ are not graphic, otherwise $N$ is p-graphic. Since $N_{2}$ is connected, there exists a circuit $C$ containing $e$ and $f$ in $N_{2}$. Then $N \backslash\left(N_{2}-C\right) /(C-\{e, f\})$ is 2-sum of $N_{1}$ and $N_{3}$ where $e$ and $f$ are regarded as the same edge. It contradicts Proposition 2.3.7 as $N_{1}$ and $N_{3}$ are both not graphic.

The following lemmas are proven respectively in [4] and [2].

Lemma 2.4.4. Let $M$ be a matroid and let $N$ be a minor of $M$. Suppose that $N$ has a unique exact 2-separation $(X, Y)$ and that $M$ is minimal major of $N$ that bridges $(X, Y)$. Then $M$ is 3-connected.

Lemma 2.4.5. If $(X, Y)$ is an exact 2-separation in a matroid $N$ and $M$ is a minimal matroid that bridges the 2-separation $(X, Y)$ in $N$, then $|E(M)|<|E(N)|+5$.

We now prove Theorem 2.1.4.

Proof of Theorem 2.1.4. Let $N$ be a binary matroid that is minimal with respect to (1) $N$ is nonp-graphic and (2) $N$ is 3-connected. Let $N^{\prime}$ be a minor of $N$ that is minimally non-p-graphic. Let $c$ be the maximum size of an excluded minor for p-graphic. By Theorem 1.1.1, $c$ is a constant. 
Note that $\left|E\left(N^{\prime}\right)\right| \leq c$. By Lemma 2.4.3, either $N^{\prime}$ is 3-connected, or $N^{\prime}$ is connected and has a unique 2-separation. If $N^{\prime}$ is 3-connected, then $N=N^{\prime}$. Thus, we may assume that $N^{\prime}$ is connected and has unique 2-separation $(X, Y)$. Let $N^{\prime \prime}$ be a minor of $N$ and a major of $N^{\prime}$ that minimally bridges $(X, Y)$. It follows from Lemma 2.4.4 that $N^{\prime \prime}$ is 3-connected. Thus, $N=N^{\prime \prime}$. By Lemma 2.4.5, $|E(N)|<\left|E\left(N^{\prime}\right)\right|+5 \leq c+5$. 


\section{Chapter 3}

\section{Recognizing p-graphic and p-cographic matroids}

The goal of this chapter is solving membership problems for p-graphic and p-cographic matroids when the given matroids are 3-connected. By Proposition 1.5.3, it suffices to have an algorithm for recognizing p-cographic matroids. In Section 3.1, we introduce folding/unfolding operations between a $B P$-representation and a $T_{4}$ representation, and prove Proposition 1.5.3. In Section 3.2, we give a brief outline of an algorithm for recognizing p-cographic matroids. Unlike other algorithms mentioned in the previous sections, this algorithm keep track of two types of classes: equivalence classes and anemone classes. Both classes and related results will be considered respectively in Section 3.3 and Section 3.4.

\subsection{From p-graphic to p-cographic}

Before we proceed to the proof for Proposition 1.5.3, we need a number of definitions from [11]. Consider a signed graph $(G, \Sigma)$ where $\Sigma \subseteq \delta_{G}(s) \cup \delta_{G}(t)$ and suppose that there are no loops and edges between $s$ and $t$. The graft $(H, T)$ obtained from $(G, \Sigma)$ by unfolding on $s, t$ is defined as follows:

(1) split $s$ into $s_{1}, s_{2}$ according to $\Sigma \cap \delta_{G}(s)$;

(2) split $t$ into $t_{1}, t_{2}$ according to $\Sigma \cap \delta_{G}(t)$;

(3) $\operatorname{set} T=\left\{s_{1}, s_{2}, t_{1}, t_{2}\right\}$. 

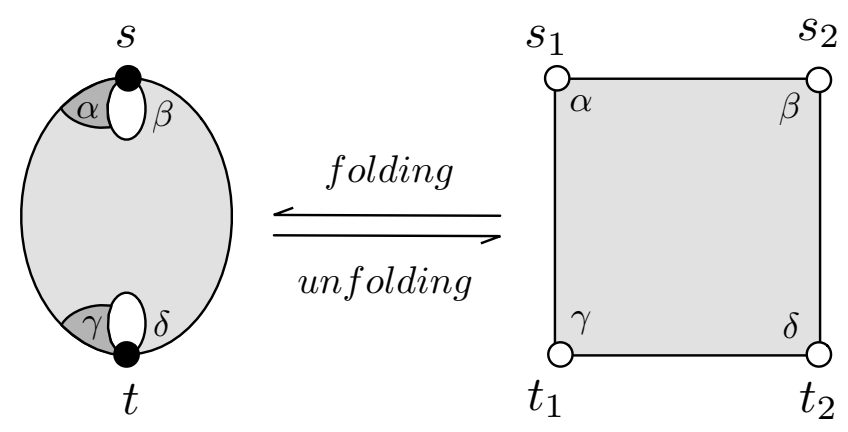

Figure 3.1: Folding/unfolding between a $B P$-representation and a $T_{4^{-}}$ representation. Shaded edges are odd and white vertices are terminals.

We illustrate the construction in the following picture, Let us extend this definition to the case where we have loops or edges $e$ with ends $s, t$ of $G$ as follows:

(a) If $e$ is an even loop in $G$ it remains an even loop at the same vertex in $H$; If $e$ is an even loop at $s$ (resp. $t$ ) in $G$, it remains an even loop at $s_{1}$ or $s_{2}$ (resp. $t_{1}$ or $t_{2}$ ) in $H$.

(b) If $e$ is an odd loop in $G$ at $s$, we have $e=\left(s_{1}, s_{2}\right)$.

(c) If $e$ is an odd loop in $G$ at $t$, we have $e=\left(t_{1}, t_{2}\right)$.

(d) If $e=(s, t)$ is even we have a choice $e=\left(s_{1}, t_{1}\right)$ or $e=\left(s_{2}, t_{2}\right)$ in $H$.

(e) If $e=(s, t)$ is odd we have a choice $e=\left(s_{1}, t_{2}\right)$ or $e=\left(s_{2}, t_{1}\right)$ in $H$.

Note that, for (a)-(e), the edge $e$ behaves similarly to a path $Q$ of length two with respect to the previous definition. For instance for (d), we think of $e$ as a path $Q$ with two odd edges or two even edges. For (e), we think of $e$ as a path $Q$ where the edge incident to $s$ is odd and the edge incident to $t$ is even or vice-versa. We say that $(G, \Sigma)$ is obtained from $(H, T)$ by folding with pairing $s_{1}, s_{2}$ and $t_{1}, t_{2}$.

The following characterization of the cocycles of even-cycle and even-cut matroids appears in [11]. We follow closely the proof given in that paper.

\section{Proposition 3.1.1.}

(1) The cocycles of ecycle $(G, \Sigma)$ are precisely cuts of $G$ and signatures of $(G, \Sigma)$; 
(2) The cocycles of ecut $(G, T)$ are precisely cycles of $G$ and $T$-joins of $G$.

Proof of Proposition 1.5.3. Let $M$ be a p-graphic matroid. By definition there exists a representation $(G, \Sigma)$ with vertices $s, t$ such that $\Sigma \subseteq \delta_{G}(s) \cup \delta_{G}(t) \cup \operatorname{loop}(G)$. Let $(H, T)=$ $\left(H,\left\{s_{1}, s_{2}, t_{1}, t_{2}\right\}\right)$ be a $T_{4}$-representation obtained by unfolding $(G, \Sigma)$ on $s, t$ where $s_{1}, s_{2}$ correspond to $s$ and $t_{1}, t_{2}$ correspond to $t$. Let $N=\operatorname{ecut}(H, T)$. We need to show that the cycles of $M$ are equal to the cocycles of $N$. Because of Proposition 3.1.1 it suffices to show that the set of all even-cycles of $(G, \Sigma)$ is equal to the union of the set of all cycles and all $T$-joins of $H$. Suppose that $C$ is an even cycle of $(H, \Gamma)$. For every $v \in V(H)-\{s, t\},\left|\delta_{H}(v) \cap C\right|=\left|\delta_{G}(v) \cap C\right|$, which is even. For $i=1,2$ define $d(s, i)=\left|C \cap \delta_{G}\left(s_{i}\right)\right|$ and $d(t, i)=\left|C \cap \delta_{G}\left(t_{i}\right)\right|$. Since $C$ is a cycle $d(s, 1), d(s, 2)$ have the same parity and so do $d(t, 1), d(t, 2)$. Note that $\alpha=\delta_{G}\left(s_{1}\right)$, $\beta=\delta_{G}\left(t_{1}\right)$ and $\Gamma=\alpha \Delta \beta$. Thus, as $|C \cap \Gamma|$ is even, $d(s, 1)$ and $d(t, 1)$ have the same parity. Thus $d(s, 1), d(s, 2), d(t, 1), d(t, 2)$ are either all even or all odd. In the former case $C$ is a cycle of $G$, in the later case it is a $T$-join of $G$. The converse is similar.

\subsection{An algorithm for recognizing p-cographic matroids}

In this section, we give a brief version of an algorithms to solve the membership problem for p-graphic(resp. p-cographic) matroids when the given matroids is 3-connected and is not graphic(resp. cographic). By Proposition 1.5.3, it is enough to solve the membership problem for p-cographic matroids. We will use the same strategy for membership problem for graphic matroids that is mentioned in Section 1.2. In section 1.3.3, the example shows that we may have exponentially many equivalence classes for a p-cographic matroid. To solve this, we will use an additional class of representations, called an anemone class. These classes will be used to consider all representations obtained by shuffling petals in Section 1.3.3 as one class of representations. The formal definition for anemone class will be introduced in Section 3.3. Recall that an equivalence class for an p-graphic matroid is given by the set of all signed graphs that are equivalent to a fixed signed graph that is a representation of that matroid. We only consider $T_{4}{ }^{-}$ representations, since p-graphic matroids are minor-closed. Let $\mathcal{A}(M)$ be obtained by selecting one $T_{4}$-representation from each anemone classes and let $\mathcal{S}(M)$ be obtained by selecting one $T_{4^{-}}$ representation from each equivalence classes that have at least one $T_{4}$-representation that is not contained in any anemone classes. The membership algorithm will keep track of $\mathcal{A}(M) \cup \mathcal{S}(M)$ for p-cographic matroids that are not cographic. Then as an analogue of Theorem 1.5.2, we have the following result,

Theorem 3.2.1. Let $M$ be a 3-connected p-cographic matroid that is not cographic. Then the 
set of all $T_{4}$-representations of $M$ is contained in a polynomial number of equivalence representations and a polynomial number of anemone classes.

Now let us outline a polynomial time algorithm for the membership problem for p-cographic matroids. Consider a 3-connected binary matroid $M$ given by a $(0,1)$-matrix representation. We check if $M$ is cographic by using the membership algorithm for cographic matroids. If $M$ is cographic, then it is also a p-cographic matroid. Moreover, the membership algorithm returns a representation $(G, T)$ where $T=\emptyset$. We can then return $(G, \emptyset)$ as a certificate and stop. Otherwise, because of Proposition 2.1.1 we can find a minor $N$ of $M$ that is minimally noncographic. Note that every minimally non-cographic matroids is 3-connected. By Theorem 1.1.1, $N$ has a bounded size. As $M$ and $N$ are 3-connected, by[13], this implies that we can construct a sequence of 3-connected matroids $M_{1}, \ldots, M_{k}$ where $M=M_{1}, M_{k}=N$, and for every $i \in[k-1], M_{i+1}$ is obtained from $M_{i}$ by deleting or contracting an element, say $e_{i}$ of $M_{i}$. Iteratively, we constructed $\mathcal{S}\left(M_{i+1}\right) \cup \mathcal{A}\left(M_{i+1}\right)$. Then, we can construct $\mathcal{S}\left(M_{i}\right) \cup \mathcal{A}\left(M_{i}\right)$ from $\mathcal{S}\left(M_{i+1}\right) \cup \mathcal{A}\left(M_{i+1}\right)$ in polynomial time in the size of $M_{i}$ and the cardinality of $\mathcal{S}\left(M_{i}\right) \cup \mathcal{A}\left(M_{i}\right)$. However, the cardinality of $\mathcal{S}\left(M_{i}\right) \cup \mathcal{A}\left(M_{i}\right)$ is bounded by a polynomial number because of Theorem 3.2.1. It follows that $\mathcal{S}\left(M_{i}\right) \cup \mathcal{A}\left(M_{i}\right)$ can be constructed in polynomial time in the size of $M_{i}$. If $\mathcal{S}\left(M_{i}\right) \cup \mathcal{A}\left(M_{i}\right)=\emptyset$ then $M_{i}$ is not a p-cographic matroid and neither is $M$. Otherwise, if $i=1$ then $M_{i}=M$ is a p-cographic matroid with representations $\mathcal{S}\left(M_{i}\right) \cup \mathcal{A}\left(M_{i}\right)$, and if $i>1$ then we iteratively construct $\mathcal{S}\left(M_{i-1}\right) \cup \mathcal{A}\left(M_{i-1}\right)$.

\subsection{Anemone classes}

\subsubsection{Flowers}

Let us review the definition of flowers from [12]. Given a matroid $M$ with rank function $r$, the connectivity function $\lambda_{M}$ returns for every $X \subseteq E(M)$ the integer, $\lambda_{M}(X):=r(X)+r(\bar{X})-$ $r(E(M))$. A flower $\Phi$ of a 3-connected matroid $M$ is an ordered partition $\left(P_{1}, \ldots, P_{n}\right)$ of $E(M)$ where $\left|P_{i}\right| \geq 2$ and $\lambda_{M}\left(P_{i}\right)=\lambda_{M}\left(P_{i} \cup P_{i+1}\right)=2$ for all $i \in[n]$ where all indices are taken modulo $n$. The sets $P_{1}, \ldots, P_{n}$ are the petals of the flower.

By a cyclic subset of $[n]$ we mean a proper subset of the form $\{i, i+1, \ldots, j-1, j\}$ with $i \leq j$ or the complement of such a set. The flower $\Phi$ is a daisy if for every cyclic subset $I$ of $[n]$, the set $\cup_{i \in I} P_{i}$ is 3 -separating (i.e. the union of consecutive petals is 3-separating) and no other union of petals is 3-separating. The flower $\Phi$ is an anemone if for every non-empty proper subset $I$ of $[n]$, the set $\cup_{i \in I} P_{i}$ is 3-separating (i.e. the union of an arbitrary set of petals is 3-separating). It is shown in [12] that for a 3-connected matroid, every flower is either a daisy or an anemone. 
Definition 3.3.1. Let $(G, T)$ be a graft with $|T|=4$. Denote by $E_{0}$ the set of edges of $G$ that have both ends in $T$. Let $P_{1}, \ldots, P_{n}$ be a partition of $E(G)-E_{0}$. The pair $\Phi=\left(E_{0},\left\{P_{1}, \ldots, P_{n}\right\}\right)$ where $n \geq 4$ is an ordinary anemone of $(G, T)$ if for every $i \in[n]$ :

(1) $G\left[P_{i}\right]$ is connected,

(2) $\mathcal{B}_{G}\left(P_{i}\right) \subseteq T$ and $\left|\mathcal{B}_{G}\left(P_{i}\right)\right| \geq 3$.

We say that $P_{1}, \ldots, P_{n}$ are the petals of the ordinary anemone and edges in $E_{0}$ are loose.

Definition 3.3.2. Let $\Phi=\left(E_{0},\left\{P_{1}, \ldots, P_{k}\right\}\right)$ be an ordinary anemone of $(G, T)$ where $T=$ $\left\{t_{1}, t_{2}, t_{3}, t_{4}\right\}$. We say that $(G, T)$ and $\left(G^{\prime}, T\right)$ are related by rearranging loose edges if $G$ can be obtained from $G^{\prime}$ by repeatedly replacing edge $e=t_{p} t_{q}$ by $e=t_{r} t_{s}$ where $\{p, q, r, s\}=[4]$. For every $i \in[n]$ let $G_{i}^{\prime}$ be the graph obtained from $G\left[P_{i}\right]$ by relabeling the terminals in one of three possible ways,

(1) interchange the labels of $t_{1}$ and $t_{2}$ the labels of $t_{3}$ and $t_{4}$;

(2) interchange the labels of $t_{1}$ and $t_{3}$ the labels of $t_{2}$ and $t_{4}$;

(3) interchange the labels of $t_{1}$ and $t_{4}$ the labels of $t_{2}$ and $t_{3}{ }^{1}$

Now let $G^{\prime}$ be obtained by identifying vertices $t_{1}\left(\right.$ resp. $\left.t_{2}, t_{3}, t_{4}\right)$ in each of $G_{i}^{\prime}$ for $i=1, \ldots, k$. We then say that $(G, T)$ and $\left(G^{\prime}, T^{\prime}\right)$ are related by rearranging petals.

In the previous definition, it can be readily checked that if $\Phi$ is an ordinary anemone for $(G, T)$ and $\left(G^{\prime}, T^{\prime}\right)$ is obtained from $(G, T)$ by rearranging petals and loose edges, then $\Phi$ is an ordinary anemone for $\left(G^{\prime}, T^{\prime}\right)$ as well. Moreover, it can be checked that $C \subseteq E(G)$ is a cycle or a $T$-join of $(G, T)$ if and only if $C$ is a cycle or a $T^{\prime}$-join of $\left(G^{\prime}, T^{\prime}\right)$. Thus, $(G, T)$ and $\left(G^{\prime}, T^{\prime}\right)$ are representations of the same p-cographic matroid. This leads to the following definition, given $\Phi$ an ordinary anemone of $(G, T)$ the set of all grafts obtained from $(G, T)$ by rearranging the loose edges or petals is the anemone class generated by $(G, T)$ and $\Phi$. Note no two members of an anemone class are related by Whitney-flips in general, i.e. are equivalent.

We saw in the introduction that ordinary anemones may give rise to p-cographic matroids that have an exponential number of pairwise inequivalent $T_{4}$-representations. In this section we gain a better understanding of these types of anemone.

\footnotetext{
${ }^{1}$ proceed as if $T \subseteq V_{G}\left(P_{i}\right)$ for all $i \in[k]$ by thinking of vertices in $T \backslash V_{G}\left(P_{i}\right)$ as isolated vertices of $G$.
} 


\subsubsection{Representations of ordinary anemones}

We will show that for a p-cographic matroid, an ordinary anemone for one representation will correspond, for another representation, to either an ordinary anemone or to a skewed anemone that we define next.

Definition 3.3.3. Let $(G, T)$ be a graft with $|T|=4$. Let $v_{1}, v_{2}, v_{3}$ be distinct vertices of $G$ and let $X=\left\{v_{1}, v_{2}, v_{3}\right\}$. Denote by $E_{0}$ as follows:

(a) if $X \subseteq T$, then let $E_{0}$ be the set of all edges that have both ends in $T$,

(b) if $|T \cap X|=2$, then let $E_{0}$ be the set of all edges that have both ends in $X$ or that have both ends in $T-X$.

(c) if $|T \cap X| \leq 1$, then let $E_{0}$ be the set of all edges that have both ends in $X$.

The pair $\Phi=\left(E_{0},\left\{P_{1}, \ldots, P_{n}\right\}\right)$ where $E_{0}, P_{1}, \ldots, P_{n}$ is a partition of $E(G)$ and where $n \geq 4$ is a skewed anemone for $(G, T)$ if

(1) $T \subseteq V_{G}\left(P_{i}\right)$ for some $i \in[n]$.

Moreover, for every $i \in[n]$ :

(2) $G\left[P_{i}\right]$ is connected, and

(3) $\mathcal{B}_{G}\left(P_{i}\right)=X$.

$P_{1}, \ldots, P_{n}$ are the petals of the skewed anemone and edges in $E_{0}$ are loose.

We illustrate the construction in Figure 3.2, Let $E$ be a set and let $\mathcal{S}=\left\{S_{1}, \ldots, S_{p}\right\}$ and $\mathcal{R}=\left\{R_{1}, \ldots, R_{q}\right\}$ be two partitions of $E$. If for every $i \in[q], R_{i} \subseteq S_{j}$ for some $j \in[p]$, then $\mathcal{R}$ is a refinement of $\mathcal{S}$. If $\mathcal{R}$ is a refinement of $\mathcal{S}$ then $\mathcal{S}$ is a coarsening of $\mathcal{R}$. An ordinary (resp. skewed) anemone $\Phi$ of a graft $(G, T)$ is maximal if no refinement $\Phi^{\prime}$ of $\Phi$ where $\left|\Phi^{\prime}\right|>|\Phi|$ is an ordinary (resp. skewed) anemone.

We are now ready to state the main result of this section,

Proposition 3.3.4. Let $M$ be a 3-connected p-cographic matroid with $T_{4}$-representations $(G, T)$ and $\left(G^{\prime}, T^{\prime}\right)$. Then $\Phi$ is an ordinary or skewed anemone of $\left(G^{\prime}, T^{\prime}\right)$ if $\Phi$ is an ordinary anemone of $(G, T)$. Moreover, if $\Phi$ is a maximal in $(G, T)$ if and only if it is maximal in $\left(G^{\prime}, T^{\prime}\right)$. 


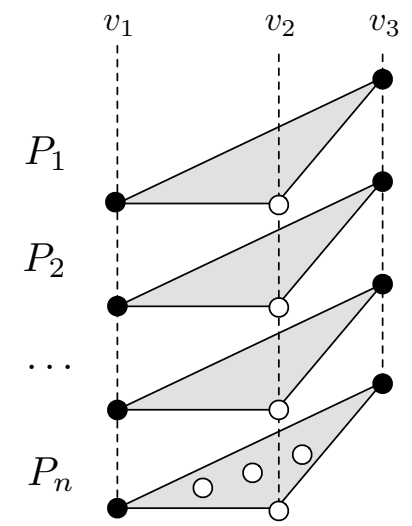

Figure 3.2: Construction of a skewed anemone. White vertices are terminals. Dotted edges mean identifying vertices.

We next define a set of matroid properties that captures the behaviour of ordinary and skewed anemones with exactly three petals. What we need is essentially the notion of a copaddle, a type of matroid anemone; however, it will be convenient in our case to modify the definition in [12] to treat separately the elements of $M$ that are in the co-span of each petal $P_{i}$. This leads to the following definition.

Definition 3.3.5. Consider a binary matroid $M$ with a partition $\left(E_{0}, P_{1}, P_{2}, \ldots, P_{n}\right)$ of $E(M)$. Let $N=M / E_{0}$. Then $\left(E_{0},\left\{P_{1}, \ldots, P_{n}\right\}\right)$ is a copaddle of $M$ if for every $i \in[n]$,

(1) $e \in E_{0}$ if and only if $e$ is a coloop of $M \backslash P_{i}$,

(2) $\lambda_{N}\left(P_{i}\right)=2$,

and for all distinct $i, j \in[n]$,

(3) $\sqcap_{N}\left(P_{i}, P_{j}\right)=0$,

(4) $\sqcap_{N^{*}}\left(P_{i}, P_{j}\right)=2$,

where $P_{1}, \ldots, P_{n}$ are the petals of the copaddle. Observe that this is related to the common notion of anemone as we can move the elements in $E_{0}$ to any petal $P_{i}$, that is,

Remark 3.3.6. Let $M$ be a 3-connected binary matroid. If $\left(E_{0},\left\{P_{1}, \ldots, P_{n}\right\}\right)$ is a copaddle of $M$, then $\left(E_{0} \cup P_{1}, \ldots, P_{n}\right)$ is an anemone of $M$. 
The proof of Proposition 3.3.4 is covered in the next three sections. In Section 3.3.2 we study 1- and 3-separations in even-cut matroids. In Section 3.3.2 we show that ordinary and skewed anemone give rise to copaddles. As a corollary we prove that ordinary and skewed anemones correspond matroid anemone. In Section 3.3.2 we show that copaddles correspond to ordinary or skewed anemone. We complete the proof of Proposition 3.3.4 in Section 3.3.2.

\section{1- and 3-separations in grafts.}

Consider a graft $(G, T)$ and $X \subset E(G)$ where $X \neq \emptyset$. We say that $X$ induces a 1-separation of Type $I$ in $(G, T)$ if $G[X], G[\bar{X}]$ are connected; $\left|\mathcal{B}_{G}(X)\right|=1$; and $T \subseteq V_{G}(X)$ or $T \subseteq$ $V_{G}(\bar{X})$. We say that $X$ induces a 1-separation of Type II in $(G, T)$ if $G[X], G[\bar{X}]$ are connected; $\left|\mathcal{B}_{G}(X)\right|=2$; and $T=\mathcal{B}_{G}(X)$.

We will need the following result about 1-separations,

Proposition 3.3.7. Let $M=\operatorname{ecut}(G, T)$ where $G$ is connected. Consider $X \subset E(G)$ where $X \neq \emptyset$ and $G[X], G[\bar{X}]$ are connected. Then $\lambda_{M}(X)=0$ if and only if $X$ induces a 1-separation of Type I or II.

Proof. Follows immediately from Proposition 2.3.3.

Consider a graft $(G, T)$ and $X \subset E(G)$ where $X \neq \emptyset$. We say that $X$ induces a 3 -separation of Type $I$ in $(G, T)$ if $G[X], G[\bar{X}]$ are connected; $\left|\mathcal{B}_{G}(X)\right|=2$; and $\mathcal{I}_{G}(X) \cap T \neq \emptyset$, and $\mathcal{I}_{G}(\bar{X}) \cap T \neq \emptyset$. We say that $X$ induces a 3-separation of Type II in $(G, T)$ if $G[X], G[\bar{X}]$ are connected; $\left|\mathcal{B}_{G}(X)\right|=3 ; T \nsubseteq \mathcal{B}_{G}(X)$; and $T \subseteq V_{G}(X)$ or $T \subseteq V_{G}(\bar{X})$. We say that $X$ induces a 3-separation of Type III in $(G, T)$ if $G[X], G[\bar{X}]$ are connected; $\left|\mathcal{B}_{G}(X)\right|=4$; and $T \subseteq \mathcal{B}_{G}(X)$ where $T \neq \emptyset$.

We will need the following result about 3-separations,

Proposition 3.3.8. Let $M=\operatorname{ecut}(G, T)$ where $G$ is connected. Consider $X \subset E(G)$ where $X \neq \emptyset$ and $G[X], G[\bar{X}]$ are connected. Then $\lambda_{M}(X)=2$ if and only if $X$ induces a 3 -separation of Type I, II or III.

Proof. Follows immediately from Proposition 2.3.3.

Proposition 3.3.9. Let $(G, T)$ be a graft with a partition $P_{1}, P_{2}, P_{3}$ of $E(G)$. Suppose that $G\left[P_{i}\right]$ and $G \backslash P_{i}$ is connected for all $i \in[3]$. Let $M=\operatorname{ecut}(G, T)$ and let $\{i, j, k\}=[3]$. Then

(1) $\lambda_{M}\left(P_{i}\right)=2$ if and only if $P_{i}$ induces a 3-separation of Type I, II, or III in $(G, T)$; 
(2) $\sqcap_{M}\left(P_{i}, P_{j}\right)=0$ if and only if $P_{i}$ induces a 1-separation of Type I or II of $(G, T) / P_{k}$;

(3) $\sqcap_{M^{*}}\left(P_{i}, P_{j}\right)=2$ if and only if $P_{i}$ induces a 3-separation of Type I, II, or III in $(G, T) \backslash P_{k}$.

Proof. (1) Follows directly from Proposition 3.3.8. (2) Remark 1.4.4 implies that $\left(G^{\prime}, T^{\prime}\right)=$ $(G, T) / P_{k}$ is a representation of $M \backslash P_{k}$. Thus $\sqcap_{M}\left(P_{i}, P_{j}\right)=\lambda_{\text {ecut }\left(G^{\prime}, T^{\prime}\right)}\left(P_{i}\right)$. By Proposition 3.3.7, $\lambda_{\text {ecut }\left(G^{\prime}, T^{\prime}\right)}\left(P_{i}\right)=0$ if and only if $P_{i}$ induces a 1-separation of Type I or II of $\left(G^{\prime}, T^{\prime}\right)$. (3) As the function $\lambda$ is invariant under duals, $\lambda_{\left(M / P_{k}\right)^{*}}\left(P_{i}\right)=\lambda_{M / P_{k}}\left(P_{i}\right)$. Remark 1.4.4 implies that $\left(G^{\prime}, T^{\prime}\right):=(G, T) \backslash P_{k}$ is a representation of $M / P_{k}$. Thus $\Pi_{M^{*}}\left(P_{i}, P_{j}\right)=\lambda_{\text {ecut }\left(G^{\prime}, T^{\prime}\right)}\left(P_{i}\right)$. By Proposition 3.3.8, $\lambda_{\text {ecut }\left(G^{\prime}, T^{\prime}\right)}\left(P_{i}\right)=2$ if and only if $P_{i}$ induces a 3 -separation of Type I, II, or III of $\left(G^{\prime}, T^{\prime}\right)$.

Proposition 3.3.10. Let $(G, T)$ be a representation of a matroid $M$ with disjoint subsets $P_{1}, P_{2}$ of $E(G)$. Suppose that $G\left[P_{i}\right]$ is connected for $i=1,2$. Let $M=\operatorname{ecut}(G, T)$. If $\sqcap_{M^{*}}\left(P_{1}, P_{2}\right)=2$ then $G\left[P_{1} \cup P_{2}\right]$ is connected.

Proof. Let $P_{3}=E(G)-\left(P_{1} \cup P_{2}\right)$. As in the proof of Proposition 3.3.9(c) we show that $\lambda_{M^{*}}\left(P_{1}, P_{2}\right)=\lambda_{\text {ecut }\left(G^{\prime}, T^{\prime}\right)}\left(P_{i}\right)$ where $\left(G^{\prime}, T^{\prime}\right)=(G, T) \backslash P_{3}$. Suppose for a contradiction $G\left[P_{1} \cup P_{2}\right]$ is not connected. As $G\left[P_{1}\right], G\left[P_{2}\right]$ are connected, $V_{G}\left(P_{1}\right) \cap V_{G}\left(P_{2}\right)=\emptyset$. But then Proposition 2.3.3 implies that $\lambda_{\text {ecut }\left(G^{\prime}, T^{\prime}\right)}\left(P_{1}\right) \leq 1$, a contradiction.

\section{From ordinary or skewed anemones to copaddles}

Proposition 3.3.11. Let $M$ be a 3-connected p-cographic matroid $M$ with a $T_{4}$-representation $(G, T)$. If $\Phi$ is an ordinary anemone of $(G, T)$, then $\Phi$ is a copaddle of $M$.

Proof. We have $\Phi=\left(E_{0},\left\{P_{1}, \ldots, P_{n}\right\}\right)$ for some partition $E_{0}, P_{1}, \ldots, P_{n}$ of $E(M)$. We need to prove properties (1)-(4) of Definition 3.3.5. By (1) and (2) in Definition 3.3.1, G[P$\left.P_{i}\right]$ is connected for $i \in[n]$. (1) $e \in E_{0}$ if and only if $e$ has both ends in $T$. Let $i \in[n]$. Remark 1.4.4 implies that $(G, T) / P_{i}$ is a representation of $M \backslash P_{i}$. By (2) in Definition 3.3.1, either $e$ is a loop of $G / P_{i}$ or $e$ is an edge of $(G, T) / P_{i}$ where the unique terminals are the ends of $e$. In either cases $e$ is a coloop of $M \backslash P_{i}$. (2) Let $i, j \in[n]$. Remark 1.4.4 implies that $(H, T):=(G, T) \backslash E_{0}$ is a representation of $N=M / E_{0}$. By Definition 3.3.1(1), $G\left[P_{i}\right]$ is connected, and by Definition 3.3.1(1) and (2), $G\left[E(M)-P_{i}\right]$ is connected. By Definition 3.3.1(2), $\mathcal{B}_{H}\left(P_{i}\right) \subseteq T$ where $\alpha:=\left|\mathcal{B}_{H}\left(P_{i}\right) \cap T\right| \in$ $\{3,4\}$. If $\alpha=3$ then $P_{i}$ induces a 3-separation of Type II in $(H, T)$. If $\alpha=4$ then $P_{i}$ induces a 3-separation of Type III in $(H, T)$. In both cases, Proposition 3.3.9(1) implies that $\lambda_{N}\left(P_{i}\right)=2$ as required. (3) Let $\alpha:=\left|\mathcal{B}_{G}\left(E(M)-P_{i}\right) \cap T\right|$. By Definition 3.3.1(2), $\alpha \in\{3,4\}$. If $\alpha=3$ then $P_{i}$ induces a 1-separation of Type II in $(H, T) /\left(E(M)-P_{i}\right)$. If $\alpha=4$ then $P_{i}$ induces a 
1-separation of Type I in $(H, T) /\left(E(M)-P_{i}\right)$. In both cases, Proposition 3.3.9(2) implies that $\sqcap_{N}\left(P_{i}, P_{j}\right)=0$ as required. (4) Let $\left(H^{\prime}, T^{\prime}\right)=(H, T) \backslash\left(E(M)-P_{i}\right)$. By Definition 3.3.1(2), $\alpha:=\left|\mathcal{B}_{G^{\prime}}\left(P_{i}\right)\right| \in\{2,3,4\}$. If $\alpha=2$ then $P_{i}$ induces a 3 -separation of Type I in $\left(G^{\prime}, T^{\prime}\right)$. If $\alpha=3$ then $P_{i}$ induces a 3-separation of Type II in $\left(G^{\prime}, T^{\prime}\right)$. If $\alpha=4$ then $P_{i}$ induces a 3separation of Type III in $\left(G^{\prime}, T^{\prime}\right)$. In all cases, Proposition 3.3.9(3) implies that $\Pi_{N^{*}}\left(P_{i}, P_{j}\right)=2$ as required.

We leave the proof of the following result as an exercise as it is very similar to that of Proposition 3.3.11.

Proposition 3.3.12. Let $M$ be a p-cographic matroid $M$ with a $T_{4}$-representation $(G, T)$. If $\Phi$ is a skewed anemone of $(G, T)$, then $\Phi$ is a copaddle of $M$.

This together with Remark 3.3.6 implies the following remark.

Remark 3.3.13. Let $M$ be a 3-connected p-cographic matroid with a $T_{4}$-representation $(G, T)$. If $\left(E_{0},\left\{P_{1}, \ldots, P_{n}\right\}\right)$ is an ordinary or a skewed anemone of $(G, T)$ then $\left(E_{0} \cup P_{1}, P_{2}, \ldots, P_{n}\right)$ is an anemone of $M$.

\section{From copaddles to ordinary or skewed anemones}

Proposition 3.3.14. Let $M$ be a 3-connected p-cographic matroid with a $T_{4}$-representation $(G, T)$. Consider a partition $E_{0}, P_{1}, \ldots, P_{n}$ of $E(M)$ where $G\left[P_{i}\right]$ is connected for every $i \in[n]$. Suppose that for every coarsening $Q_{1}, Q_{2}, Q_{3}$ of $P_{1}, \ldots, P_{n},\left(E_{0},\left\{Q_{1}, Q_{2}, Q_{3}\right\}\right)$ is a copaddle of $M$. Then $\Phi=\left(E_{0},\left\{P_{1}, \ldots, P_{n}\right\}\right)$ is an ordinary or a skewed anemone of $(G, T)$.

We will proceed by induction on the number of petals. Let us first consider the base case where we have exactly three petals.

Proposition 3.3.15. Let $M$ be a 3-connected p-cographic matroid that is not cographic. Let $(G, T)$ be a $T_{4}$-representation of $M$. If $\Phi=\left(E_{0},\left\{Q_{1}, Q_{2}, Q_{3}\right\}\right)$ is a copaddle of $M$ and $G\left[Q_{i}\right]$ is connected for all $i \in[3]$ then $\Phi$ is an ordinary or a skewed anemone of $(G, T)$.

Proof. Let $H=G \backslash E_{0}$. Remark 1.4.4 implies that $(H, T)$ is a representation of $N=M / E_{0}$.

\section{Claim 1.}

(a) If $\mathcal{B}_{G}\left(Q_{i}\right) \subseteq T$ for all $i \in[3]$, then $\Phi$ is an ordinary anemone. 
(b) If $T \subseteq V_{G}\left(Q_{i}\right)$ for some $i \in[3]$ and there exists $a, b, c \in V(G)$ such that for all $i \in$ [3], $\mathcal{B}_{G}\left(Q_{i}\right) \subseteq\{a, b, c\}$, then $\Phi$ is a skewed anemone.

Proof. (a) Let $e \in E_{0}$ and let $i \in$ [3]. Since $e$ is a coloop of $M \backslash Q_{i}$ it is either a loop of $G / Q_{i}$ or an edge of $(G, T) / Q_{i}$ where the only terminals are then end of $e$. Since this holds for all $i \in[3]$ and since by hypothesis $\mathcal{B}_{G}\left(Q_{i}\right) \subseteq T$ the ends of $e$ must be in $T$. We need to show that each property (1) and (2) of Definition 3.3.1 holds. (1) holds by hypothesis, (2) by hypothesis $\mathcal{B}_{G}\left(Q_{i}\right) \subseteq T$. By Definition 3.3.5(4), $\lambda_{M}\left(Q_{i}\right)=2$. By Proposition 3.3.9(1) $Q_{i}$ induces a 3separation of Type II, or III in $(H, T)$. It follows that $\left|\mathcal{B}_{H}\left(Q_{3}\right) \cap T\right| \in\{3,4\}$ as required. (b) Similarly as in (a) we prove that edges in $E_{0}$ have both ends in $\{a, b, c\}$. We need to show that each property (1)-(3) of Definition 3.3.3 holds. (1) by hypothesis, (2) by Claim 1. The proof for (3) is similar to (2) of part (a).

Consider first the case where for some $i \in[3], T \cap \mathcal{I}_{H}\left(Q_{i}\right) \neq \emptyset$. We may assume $i=3$. Let $\left(H^{\prime}, T^{\prime}\right)=(H, T) \backslash Q_{3}$. As $T \cap \mathcal{I}_{H}\left(Q_{3}\right) \neq \emptyset, T^{\prime}=\emptyset$. By Definition 3.3.5(4), $\sqcap_{M^{*}}\left(Q_{1}, Q_{2}\right)=2$. Equivalently, by Proposition 3.3.9(3) $Q_{1}$ induces a 3-separation of Type I, II, or III in $\left(H^{\prime}, T^{\prime}\right)$. But as $T^{\prime}=\emptyset$ it is must be of Type II. Denote by $a, b, c$ the vertices in $\mathcal{B}_{H^{\prime}}\left(Q_{1}\right)$. Thus $V_{H}\left(Q_{1}\right) \cap$ $V_{H}\left(Q_{2}\right)=\{a, b, c\}$. By Definition 3.3.5(2), $\lambda_{M}\left(Q_{1}\right)=2$. By Proposition 3.3.9(1) $Q_{1}$ induces a 3-separation of Type I, II, or III in $(H, T)$. Since $\mathcal{B}_{H}\left(Q_{1}\right) \subseteq\{a, b, c\}$ and since $T \cap \mathcal{I}_{H}\left(Q_{3}\right) \backslash$ $V\left(Q_{1}\right) \neq \emptyset$, it must be of Type II. Thus $\mathcal{B}_{H}\left(Q_{1}\right)=\{a, b, c\}$ and $T \cap \mathcal{I}_{H}\left(Q_{1}\right)=\emptyset$. Similarly, $\mathcal{B}_{H}\left(Q_{2}\right)=\{a, b, c\}$ and $T \cap \mathcal{I}_{H}\left(Q_{2}\right)=\emptyset$. Thus $\mathcal{B}_{H}\left(Q_{3}\right) \subseteq\{a, b, c\}$ and $T \subseteq V_{H}\left(Q_{3}\right)$. It then follows from the Claim that $\Phi$ is a skewed anemone of $(G, T)$.

Thus we will assume that,

$$
\mathcal{I}_{H}\left(Q_{i}\right) \cap T=\emptyset \quad \text { for all } i \in[3] .
$$

Let $\left(H^{\prime}, T^{\prime}\right):=(H, T) / Q_{3}$. By Definition 3.3.5(3), $\sqcap_{N}\left(Q_{1}, Q_{2}\right)=0$. By Proposition 3.3.9(2), Case 1 or Case 2 occurs.

Case 1. $Q_{1}$ (resp. $\left.Q_{2}\right)$ induces a 1 -separation of $\left(H^{\prime}, T^{\prime}\right)$ of Type I.

It follows that $\mathcal{B}_{H}\left(Q_{i}\right) \subseteq \mathcal{B}_{H}\left(Q_{3}\right)$ for $i=1,2$. Because of (3.1), $T^{\prime}=\emptyset$ thus $T \subseteq V_{H}\left(Q_{3}\right)$. But then (3.1) implies in fact that $T \subseteq \mathcal{B}_{H}\left(Q_{3}\right)$. Proposition 3.3.10 implies that $H\left[Q_{1} \cup Q_{2}\right]$ is connected. By Definition 3.3.5(2), $\lambda_{M}\left(Q_{3}\right)=2$. By Proposition 3.3.9(a) $Q_{3}$ induces a 3separation of Type I, II, or III in $(H, T)$. As $T \subseteq \mathcal{B}_{H}\left(Q_{3}\right)$ it must be of Type III. Since $M$ is a pcographic matroid that is not cographic, $|T|=4$ and $\mathcal{B}_{H}\left(Q_{3}\right)=T$. Then $\mathcal{B}_{H}\left(Q_{i}\right) \subseteq \mathcal{B}_{H}\left(Q_{3}\right)=$ $T$ for $i=1,2$. By Definition 3.3.5(2), $\lambda_{M}\left(Q_{i}\right)=2$ for $i=1,2$. By Proposition 3.3.9(1) $Q_{i}$ induces a 3-separation of Type I, II, or III in $(H, T)$. As $\mathcal{I}_{H}\left(Q_{i}\right) \cap T=\emptyset$, it must be of Type II or III. In all cases it follows from the Claim that $\Phi$ is an ordinary anemone for $(H, T)$. 
Case 2. $Q_{1}$ (resp. $\left.Q_{2}\right)$ induces a 1-separation of $\left(H^{\prime}, T^{\prime}\right)$ of Type II.

Denote by $t_{1}, t_{2}, t_{3}, t_{4}$ the vertices in $T$. Then we have, say, $t_{1}$ which is the unique vertex in $V_{H}\left(Q_{1}\right) \cap V_{H}\left(Q_{2}\right)-V_{H}\left(Q_{3}\right)$, and $t_{2}, t_{3}, t_{4} \in \mathcal{B}_{H}\left(Q_{3}\right)$. Because of $t_{1}, H\left[Q_{1} \cup Q_{2}\right]$ is connected. By Definition 3.3.5(2), $\lambda_{M}\left(Q_{3}\right)=2$. By Proposition 3.3.9(1) $Q_{3}$ induces a 3-separation of Type I, II, or III in $(H, T)$. Because of $t_{1}$ it is not of Type III. Because of $t_{2}, t_{3}, t_{4}$ it is not of Type I, thus it is of Type II, i.e. $\mathcal{B}_{H}\left(Q_{3}\right)=\left\{t_{2}, t_{3}, t_{4}\right\}$. By Definition 3.3.5(2), $\lambda_{M}\left(Q_{i}\right)=2$ for $i=1,2$. By Proposition 3.3.9(1) $Q_{i}$ induces a 3-separation of Type I, II, or III in $(H, T)$. As $\mathcal{I}_{H}\left(Q_{i}\right) \cap T=\emptyset$, it must be of Type II or III. In all cases it follows from the Claim that $\Phi$ is an ordinary anemone for $(H, T)$.

We now generalize the previous result to the case where we have an arbitrary number of petals.

Proof of Proposition 3.3.14. We have $\Phi=\left(E_{0},\left\{P_{1}, \ldots, P_{n}\right\}\right)$. Let us proceed by induction on $n$. We may assume $n \geq 4$ for otherwise the result follows from Proposition 3.3.15. Define $\Phi^{+}(i, j)$ as the partition $\Phi-\left\{P_{i}, P_{j}\right\} \cup\left\{P_{i} \cup P_{j}\right\}$ and define $\Phi^{-}(i, j)$ as the partition $\cup_{\ell \in[k]-\{i, j\}} P_{\ell}, P_{i}, P_{j}$. Observe that if $\left\{Q_{1}, Q_{2}, Q_{3}\right\}$ is a coarsening of $\Phi^{+}(i, j)$ (resp. $\Phi^{-}(i, j)$ ) then it is also a coarsening of $\Phi$. Moreover, if the petals $G\left[P_{i}\right]$ are connected in $G$ then so are the petals in any coarsening. Thus the hypothesis of the theorem also hold for $\Phi^{+}(i, j)$ and $\Phi^{-}(i, j)$.

For all distinct $i, j \in[k]$ it follows by induction that $\Phi^{+}(i, j)$ is an ordinary or skewed anemone of $(G, T)$ and that $\Phi^{-}(i, j)$ is an ordinary or skewed anemone of $(G, T)$. Consider first the case where $\mathcal{I}_{G}\left(P_{i}\right) \cap T=\emptyset$ for all $i \in[k]$. Pick distinct $i, j \in[k]$. Then $\Phi^{+}(i, j)$ and $\Phi^{+}(i, j)$ are both ordinary anemones of $(G, T)$. It follows that for all $P \in \Phi^{+}(i, j) \cup \Phi^{-}(i, j)$, $\mathcal{B}_{G}(P) \subseteq T$ and $\left|\mathcal{B}_{G}(P) \cap T\right| \in\{3,4\}$. In particular, this holds for all $P=P_{i}$ with $i=1, \ldots, k$, hence $\Phi$ is an ordinary anemone. Consider the case where $\mathcal{I}_{G}\left(P_{i}\right) \cap T \neq \emptyset$ for some $i \in[k]$. Pick $j \in[k]-\{i\}$. Then $\Phi^{+}(i, j)$ and $\Phi^{+}(i, j)$ are both skewed anemones of $(G, T)$. It follows that there exists $a, b, c \in V(G)$ such that for all $P \in \Phi^{+}(i, j) \cup \Phi^{-}(i, j), \mathcal{B}_{G}(P)=\{a, b, c\}$. In particular, this holds for all $P=P_{i}$ with $i=1, \ldots, k$, hence $\Phi$ is a skewed anemone.

\section{The proof of Proposition 3.3.4}

Let $M$ be a matroid and let $X, Y \subseteq E(M)$. We say that $X$ and $Y$ are crossing if none of $X \cap Y$, $X \cap \bar{Y}, \bar{X} \cap Y$ and $\bar{X} \cap \bar{Y}$ is an empty set.

Lemma 3.3.16. Let $M$ be a 3-connected p-cographic matroid with $T_{4}$-representations $(G, T)$ and $\left(G^{\prime}, T^{\prime}\right)$. Let $\Phi=\left(E_{0},\left\{P_{1}, \ldots, P_{n}\right\}\right)$ be an ordinary anemone of $(G, T)$. Then for all $i \in[n], G^{\prime}\left[E(M)-P_{i}\right]$ is connected. 
Proof. Suppose not. Then there exists $X \subseteq E(M)-P_{i}$ such that $(X, \bar{X})$ is a 1- or 2-separation of $M^{\prime}=M \backslash P_{i}$. Thus, $\lambda_{M^{\prime}}(X) \leq 1$. Suppose that for every $i \in[n], X$ and $P_{i}$ are not crossing. Then, by taking complement of $X$ if necessary, we may assume $X \subseteq P_{j}$ for some $j \in[n]-\{i\}$. Then $\lambda_{M}(X)=\lambda_{M^{\prime}}(X) \leq 1$, a contradiction. Thus, $X$ and $P_{j}$ are crossing for some $j \in$ $[n]-\{i\}$. Then by submodularity, $\lambda_{M^{\prime}}\left(X \cap P_{j}\right)+\lambda_{M^{\prime}}\left(X \cup P_{j}\right) \leq \lambda_{M^{\prime}}(X)+\lambda_{M^{\prime}}\left(P_{j}\right) \leq 3$. Thus, $\lambda_{M^{\prime}}\left(X \cap P_{j}\right) \leq 1$ or $\lambda_{M^{\prime}}\left(X \cup P_{j}\right) \leq 1$. Since $M$ is 3 -connected, $X \cap P_{j}$ or $\bar{X} \cap \bar{P}_{j}$ is an edge. By the similar argument, $X \cap \bar{P}_{j}$ or $\bar{X} \cap P_{j}$ is an edge. If $\bar{X} \cap P_{j}$ is an edge, then $\left|P_{j}\right|=2$. This contradicts that $\left|\mathcal{B}_{G}\left(P_{j}\right)\right| \geq 3$. Thus, $X \cap \bar{P}_{j}$ is an edge. Thus, for some $k \in[n]-\{i, j\}$, $X \cap P_{k} \neq \emptyset$ and $\mathcal{I}_{G}(X) \cap T \neq \emptyset$. Thus, $\lambda_{M^{\prime}}(X)=2$, giving a contradiction.

We are now ready for the proof of the main result of this section.

Proof of Proposition 3.3.4. Let $\Phi=\left(E_{0},\left\{P_{1}, \ldots, P_{n}\right\}\right)$ be an ordinary anemone of $(G, T)$.

Claim 1. $G^{\prime}\left[P_{i}\right]$ is connected for all $i \in[n]$.

Proof. Suppose there exists $i \in[n]$ such that $G^{\prime}\left[P_{i}\right]$ is not connected. Let $C_{1}, \ldots, C_{k}$ be components of $G^{\prime}\left[P_{i}\right]$. By Proposition 2.3.3, $\lambda\left(P_{i}\right)=\left|\mathcal{B}_{G^{\prime}}\left(P_{i}\right)\right|+p_{1}+p_{2}-k-1$ where $p_{1}, p_{2}$ are corresponding constant in Proposition 2.3.3. Since $M$ is 3-connected, $\left|\mathcal{B}_{G^{\prime}}\left(C_{j}\right)\right| \leq 2$ for all $j=1, \ldots, k$. Suppose $k \leq 4$. Then, $\lambda\left(P_{i}\right) \leq\left|\mathcal{B}_{G^{\prime}}\left(P_{i}\right)\right|-k-1 \leq 3$ contradicting $\lambda\left(P_{i}\right)=2$. If $k=3$, then $\left|\mathcal{B}_{G^{\prime}}\left(C_{j}\right)\right|=2$ for all $j=1,2,3$ and $p_{1}=p_{2}=0$. Since $M$ is 3 -connected $\mathcal{I}_{G^{\prime}}\left(C_{j}\right) \neq \emptyset$, so $p_{1}=1$, giving a contradiction. Thus, $k=2$. If $\left|\mathcal{B}_{G^{\prime}}\left(P_{i}\right)\right|=5$, then $\left|\mathcal{B}_{G^{\prime}}\left(C_{j}\right)\right|=2$ for some $j \in[2]$ and $p_{1}=p_{2}=0$, but by the similar argument, $p_{1}=1$, giving a contradiction. Thus, $\left|\mathcal{B}_{G^{\prime}}\left(P_{i}\right)\right|=4,\left|\mathcal{B}_{G^{\prime}}\left(C_{j}\right)\right|=2, p_{1}=1$ and $p_{2}=0$. Since $M$ is 3-connected, $\mathcal{I}_{G^{\prime}}\left(C_{j}\right) \neq \emptyset$ for $j=1,2$ and $\mathcal{I}_{G^{\prime}}\left(\bar{P}_{i}\right)=\emptyset$. Thus, $P_{i}$ is the unique petal such that $G^{\prime}\left[P_{i}\right]$ is not connected. Then we can use the same argument in the proof of Proposition 3.3.15 to show that there exists $a, b, c \in V\left(G^{\prime}\right)$ such that for all $j \in[n]$ but $i, \mathcal{B}_{G^{\prime}}\left(P_{j}\right)=\{a, b, c\}$. By Proposition 3.3.16, $\mathcal{B}_{G^{\prime}}\left(C_{j}\right) \cap\{a, b, c\} \neq \emptyset$ for $j=1,2$. We may assume $\left|\mathcal{B}_{G^{\prime}}\left(C_{1}\right)\right|=1$. Thus, there exists $m \in[n]$ such that $\mathcal{B}_{G^{\prime}}\left(C_{j}\right) \subseteq V_{G^{\prime}}\left(P_{m}\right)$. Then $\lambda\left(P_{i}, P_{m}\right) \leq 1$, giving a contradiction. $\diamond$

Let $Q_{1}, Q_{2}, Q_{3}$ be a coarsening of $P_{1}, \ldots, P_{n}$ and let $\Phi^{\prime}=\left(E_{0},\left\{Q_{1}, Q_{2}, Q_{3}\right\}\right)$. If $\Phi^{\prime}$ is an ordinary anemone of $(G, T)$ then by Proposition 3.3.11 $\Phi^{\prime}$ is a copaddle. If $\Phi^{\prime}$ is a skewed anemone of $(G, T)$ then by Proposition 3.3.12 $\Phi^{\prime}$ is a copaddle. It follows from Claim 2 and Proposition 3.3.14 that $\Phi$ is an ordinary or a skewed anemone of $\left(G^{\prime}, T^{\prime}\right)$. Suppose for a contradiction, $\Phi$ is maximal in $(G, T)$ but that $\Phi$ is not maximal in $\left(G^{\prime}, T^{\prime}\right)$. Then there is a refinement $\hat{\Phi}$ of $\Phi$ that is an ordinary or skewed anemone of $\left(G^{\prime}, T^{\prime}\right)$. Applying the first part of the theorem it follows that $\hat{\Phi}$ is an ordinary or skewed anemone of $(G, T)$. Observe that $\Phi$ is an ordinary 
(resp. skewed) anemone of $(G, T)$ if and only if $\hat{\Phi}$ is an ordinary (resp. skewed) anemone of $(G, T)$, contradicting the fact that $\Phi$ is maximal.

\subsubsection{Ordinary anemones related by arranging petals and loose edges}

If $\Phi$ is an ordinary anemone for two $T_{4}$-representations of a p-cographic matroid, then we can describe exactly the relationship between these representations. The analogous statement where we replace ordinary anemones by skewed ones is not true.

Proposition 3.3.17. Let $M$ be a 3-connected p-cographic matroid that is not cographic. Let $(G, T)$ and $\left(G^{\prime}, T^{\prime}\right)$ be $T_{4}$-representations of $M$. If $\Phi=\left(E_{0},\left\{P_{1}, \ldots, P_{n}\right\}\right)$ is a maximal ordinary anemone of both $(G, T)$ and $\left(G^{\prime}, T^{\prime}\right)$, then $(G, T),\left(G^{\prime}, T^{\prime}\right)$ are related by rearranging petals and loose edges.

Proof. Let $i \in[n]$. Let $H$ be obtained from $G\left[P_{i}\right]$ by identifying vertices in $\mathcal{B}_{G}\left(P_{i}\right)$ and let $H^{\prime}$ be obtained from $G^{\prime}\left[P_{i}\right]$ by identifying vertices in $\mathcal{B}_{G^{\prime}}\left(P_{i}\right)$.

Claim 1. $H$ and $H^{\prime}$ are equivalent.

Proof. Let $D=E(M)-P_{i}$. By Definition 3.3.1(2), $\alpha:=\left|\mathcal{B}_{G}\left(P_{i}\right)\right| \in\{3,4\}$. Consider first the case where $\alpha=4$. Then $H=G / D$, and $T \subseteq V_{G}(D)$. In particular, $(G, T) / D=(H, \emptyset)$. It follows that

$$
\operatorname{ecut}(G, T) \backslash D=\operatorname{ecut}((G, T) / D)=\operatorname{ecut}(H, \emptyset)=\operatorname{cut}(H)
$$

Consider now the case where $\alpha=3$, say $\mathcal{B}_{G}(G)=\left\{t_{1}, t_{2}, t_{3}\right\}$ where $T=\left\{t_{1}, t_{2}, t_{3}, t_{4}\right\}$. Denote by $\hat{H}$ the graph obtained $G\left[P_{i}\right]$ by identifying $t_{1}, t_{2}, t_{3}$ to a single vertex, say $\hat{t}$. Then $\hat{H}=G / D$. Note that $H$ is obtained from $\hat{H}$ by identifying $\hat{t}$ and $t_{4}$. It follows that,

$$
\operatorname{ecut}(G, T) \backslash D=\operatorname{ecut}((G, T) / D)=\operatorname{ecut}\left(\left(\hat{H},\left\{\hat{t}, t_{4}\right\}\right)=\operatorname{cut}(H)\right.
$$

where the first equation holds by Remark 1.4.4. Thus in both cases $\operatorname{cut}(H)=\operatorname{ecut}(G, T) \backslash D$. Similarly, for $G^{\prime}$ we show that $\operatorname{cut}\left(H^{\prime}\right)=\operatorname{ecut}\left(G^{\prime}, T^{\prime}\right) \backslash D$. It follows that $\operatorname{cut}(H)=\operatorname{cut}\left(H^{\prime}\right)$, or equivalently, $\operatorname{cycle}(H)=\operatorname{cycle}\left(H^{\prime}\right)$. By Theorem 1.2.3 this implies that $H$ and $H^{\prime}$ are related by Whitney-flips. Thus, $H$ and $H^{\prime}$ are equivalent.

Claim 2. $H$ and $H^{\prime}$ are isomorphic.

Proof. By Claim 1, $H$ and $H^{\prime}$ are equivalent. Suppose $H$ and $H^{\prime}$ are not isomorphic. Suppose there is a 2-separation $\left(X, P_{i}-X\right)$ of $H$ that is also a 2-separation of $G$. By Proposition 2.3.3, $\lambda_{M}(X)=1$. This contradicts 3 -connectivity of $M$. Thus, every 2 -separation of $H$ has the 
identified vertex as its one of boundary vertices and it is a 3-,4- or 5-separation in $G$. Thus, no two 2-separations in the sequence of Whitney-flips from $H$ to $H^{\prime}$ are crossing. Let $\left(X, P_{i}-X\right)$ be one of 2-separations that is performed from $H$ to $H^{\prime}$. Then, $\left(X, P_{i}-X\right)$ is also a 2-separation of $H^{\prime}$. By rearranging petals if necessary, we may assume there exist two petals $P_{j}, P_{k}$ where $i, j, k$ are all distinct and $\mathcal{B}_{G}\left(P_{j}\right) \cap \mathcal{B}_{G}\left(P_{k}\right)=T$. Then we can find a path $Q_{1}$ in $G\left[P_{j}\right]$ and a path $Q_{2}$ in $G\left[P_{k}\right]$, such that $Q_{1} \cup Q_{2}$ is a $T$-join of $(G, T)$. Let $Q=Q_{1} \cup Q_{2}$ and let $R=\bar{P}_{i}-Q$. In fact, there are at least two choices for the pair $\left(Q_{1}, Q_{2}\right)$, thus we may assume that in $G / Q \backslash R$, $\left(X, P_{i}-X\right)$ is a 3-separation. Since $Q$ is a cocircuit of ecut $(G, T), Q$ is either a $T^{\prime}$-join of $\left(G^{\prime}, T^{\prime}\right)$ or a circuit of $G^{\prime}$. Consider first the case where $Q$ is a $T^{\prime}$-join of $\left(G^{\prime}, T^{\prime}\right)$. Let $F=G / Q \backslash R$ and let $F^{\prime}=G^{\prime} / Q \backslash R$. Then,

$$
\operatorname{cut}(F)=\operatorname{ecut}(F, \emptyset)=\operatorname{ecut}(G, T) / Q \backslash R=\operatorname{ecut}\left(G^{\prime}, T^{\prime}\right) / Q \backslash R=\operatorname{ecut}\left(F^{\prime}, \emptyset\right)=\operatorname{cut}\left(F^{\prime}\right)
$$

By Theorem 1.2.3, $F$ and $F^{\prime}$ are related by Whitney-flips, giving a contradiction. Consider now the case where $Q$ is a circuit of $G^{\prime}$. Note that $V_{G^{\prime}}(Q) \cap T \geq 2$, otherwise $Q$ is a disjoint union of two circuits in ecut $(G, T) / P_{i}$, but $Q$ is a circuit in ecut $\left(G^{\prime}, T^{\prime}\right) / P_{i}$, giving a contradiction. Let $F=G / Q \backslash R$ and let $F^{\prime}=G^{\prime} / Q \backslash R$. Note that in ecut $\left(G^{\prime}, T^{\prime}\right) / Q \backslash R$, there are at most two terminals. By identifying possible two terminals, we may assume that ecut $\left(G^{\prime}, T^{\prime}\right) / Q \backslash R$ has no terminals. Then,

$$
\operatorname{cut}(F)=\operatorname{ecut}(F, \emptyset)=\operatorname{ecut}(G, T) / Q \backslash R=\operatorname{ecut}\left(G^{\prime}, T^{\prime}\right) / Q \backslash R=\operatorname{ecut}\left(F^{\prime}, \emptyset\right)=\operatorname{cut}\left(F^{\prime}\right)
$$

By Theorem 1.2.3, $F$ and $F^{\prime}$ are related by Whitney-flips, giving a contradiction.

Claim 3. Let $i \in[n]$ then $G\left[P_{i}\right]$ and $G^{\prime}\left[P_{i}\right]$ are isomorphic.

Proof. Suppose for a contradiction that $G\left[P_{i}\right]$ and $G^{\prime}\left[P_{i}\right]$ are not isomorphic. It follows from Claim 1 that we must have edges $e_{1}, e_{2} \in P_{i}$ such that in $G, e_{j}$ is incident to $t_{j}$ for $j=1,2$ where $t_{1}, t_{2}$ are distinct elements of $T$, and in $G^{\prime}, e_{1}, e_{2}$ are incident to the same vertex $t^{\prime} \in T^{\prime}$. Since $\Phi$ is maximal, $G\left[P_{i}\right] \backslash \mathcal{B}\left(P_{i}\right)$ is connected. It follows that there exists a path $Q$ in $G\left[P_{i}\right] \backslash \mathcal{B}\left(P_{i}\right)$ such that $Q \cup\left\{e_{1}, e_{2}\right\}$ forms a $t_{1} t_{2}$-path in $G\left[P_{i}\right]$. But then Claim 1 implies that $Q \cup\left\{e_{1}, e_{2}\right\}$ forms a circuit in $G^{\prime}\left[P_{i}\right]$. By Proposition 3.1.1 it implies that $Q \cup\left\{e_{1}, e_{2}\right\}$ is a cocircuit of $M$. But, then, Proposition 3.1.1 implies that $Q \cup\left\{e_{1}, e_{2}\right\}$ is either a circuit of $G$ or a $T$-join of $G$, a contradiction as $Q \cup\left\{e_{1}, e_{2}\right\}$ is a path of $G$ and $|T|=4$.

By a tripod of $G\left[P_{i}\right]$ (resp. $G^{\prime}\left[P_{i}\right]$ ) we mean a triple $\left(Q_{1}, Q_{2}, Q_{3}\right)$ where $Q_{1}, Q_{2}, Q_{3}$ are three edge disjoint paths in $G$ (resp. $G^{\prime}$ ) that share exactly one vertex $v_{0} \in \mathcal{I}_{G}\left(P_{i}\right)$ as an initial vertex and where the other end of $Q_{j}$, for $j=1,2,3$ is a vertex in $T$.

Claim 4. Let $i \in[n]$. Then there exists a tripod $\left(Q_{1}, Q_{2}, Q_{3}\right)$ of $G\left[P_{i}\right]$ (resp. $\left.G^{\prime}\left[P_{i}\right]\right)$ and distinct elements $t_{1}, t_{2}, t_{3} \in T$ where $t_{j}$ is the end of $Q_{j}$ for $j=1,2,3$. 
Proof. Since $\Phi$ is maximal, $G\left[P_{i}\right] \backslash \mathcal{B}\left(P_{i}\right)$ is connected. It follows that there exists in $G$ : a $t_{1}, t_{2^{-}}$ path $L \subseteq P_{i}$ that is internally disjoint from $T$, and a path $L^{\prime}$ with end in $V_{G}(L)-T$ and $t_{3}$ that is internally disjoint from $L$. Then $L \cup L^{\prime}$ contain the edges of the required tripod. Similarly, we can find the tripod in $G^{\prime}\left[P_{i}\right]$.

Since $M$ is not cographic, there exists $j \in[n]$, say $j=1$, such that $\mathcal{B}_{G}\left(P_{j}\right)=T$ (for otherwise we can rearrange the petals to get another representation of $M$ where a terminal becomes an isolated vertex, which implies that $M$ is cographic). Denote by $t_{1}, t_{2}, t_{3}, t_{4}$ the elements in $T$ and $T^{\prime}$. Recall from Claim 2, that $G\left[P_{i}\right]$ and $G^{\prime}\left[P_{i}\right]$ are isomorphic for all $i \in[n]$. We may assume that $G\left[P_{1}\right]$ are $G\left[P_{2}\right]$ are identical, in particular that vertex $t_{j}$ of $G\left[P_{1}\right]$ corresponds to vertex $t_{j}$ of $G^{\prime}\left[P_{1}\right]$ for all $j=1, \ldots, 4$. It can be readily checked that edges of $E_{0}$ in $G$ and $G^{\prime}$ are related by rearranging edges. We need to show that $G \backslash E_{0}$ are $G^{\prime} \backslash E_{0}$ are related by rearranging petals. Let $i \in\{2, \ldots, n\}$. It suffices to show that $G^{\prime}\left[P_{i}\right]$ is obtained from $G\left[P_{i}\right]$ by either:

i. keeping same labels for vertices of $G\left[P_{i}\right]$ and $G^{\prime}\left[P_{i}\right]$; or

ii. interchanging the labels of $t_{1}$ and $t_{2} \&$ the labels of $t_{3}$ and $t_{4}$; or

iii. interchanging the labels of $t_{1}$ and $t_{3} \&$ the labels of $t_{2}$ and $t_{4}$; or

iv. interchanging the labels of $t_{1}$ and $t_{4} \&$ the labels of $t_{2}$ and $t_{3}{ }^{2}$

By Claim 3 there exists a tripod $\left(Q_{1}, Q_{2}, Q_{3}\right)$ of $G\left[P_{1}\right]$ where $t_{j}$ is the end of $Q_{j}$ for $j=1,2,3$. We may assume (after possibly relabeling the vertices of $T$ in both $G$ and $G^{\prime}$ that $\mathcal{B}_{G}\left(P_{i}\right) \supseteq$ $\left\{t_{1}, t_{2}, t_{3}\right\}$. By Claim 3 there exists a tripod $\left(R_{1}, R_{2}, R_{3}\right)$ of $G\left[P_{i}\right]$ where $t_{j}$ is the end of $R_{j}$ for $j=1,2,3$. By Claim $2\left(Q_{1}, Q_{2}, Q_{3}\right)$ is a tripod of $G^{\prime}\left[P_{1}\right]$ and $\left(R_{1}, R_{2}, R_{3}\right)$ is a tripod of $G^{\prime}\left[P_{i}\right]$. Denote by $C(i, j)$ the set $Q_{i} \cup Q_{j} \cup R_{i} \cup R_{j}$. Exactly one of the following cases 1-4 occurs.

Case 1: $R_{1}$ has end $t_{1}$ in $G^{\prime} . C(1,2)$ is a circuit of $G$. By Proposition 3.1.1 it is either a circuit of a $T^{\prime}$-join of $G^{\prime}$, but it cannot be the latter, hence it is a circuit. It follows that $R_{2}$ has end $t_{2}$ in $G^{\prime}$. By considering $C(1,3)$ we prove similarly that $R_{3}$ has end $t_{3}$ in $G^{\prime}$. Hence, (i) occurs.

Case 2: $R_{2}$ has end $t_{1}$ in $G^{\prime} . C(1,2)$ is a circuit of $G$. By Proposition 3.1.1 it is either a circuit of a $T^{\prime}$-join of $G^{\prime}$, but it cannot be the latter, hence it is a circuit. It follows that $R_{2}$ has end $t_{1}$ in $G^{\prime}$. $C(1,3)$ is a circuit of $G$. By Proposition 3.1.1 it is either a circuit of a $T^{\prime}$-join of $G^{\prime}$. But it cannot be the former, hence it is a $T^{\prime}$-join. It follows that $R_{3}$ has end $t_{4}$ in $G^{\prime}$. Hence, (ii) occurs.

Case 3: $R_{3}$ has end $t_{1}$ in $G^{\prime}$. Similarly, as in the previous case, we have that $R_{3}$ has end $t_{1}$ in $G^{\prime}, R_{1}$ has end $t_{3}$ in $G^{\prime}$ and $R_{2}$ has end $t_{4}$ in $G^{\prime}$. Hence, outcome (iii) occurs.

\footnotetext{
${ }^{2}$ If $T \nsubseteq \mathcal{B}_{G}\left(P_{i}\right)$ then for the purpose of relabeling we think of the vertex in $T-\mathcal{B}_{G}\left(P_{i}\right)$ as part of $G\left[P_{i}\right]$.
} 
Case 4: none of $R_{1}, R_{2}, R_{3}$ has end $t_{1}$ in $G^{\prime} . C(2,3)$ is a circuit of $G$. By Proposition 3.1.1 it is either a circuit of a $T^{\prime}$-join of $G^{\prime}$, but it cannot be the latter, hence it is a circuit. It follows that $R_{2}, R_{3}$ have ends $t_{2}, t_{3}$ or $t_{3}, t_{2}$ respectively in $G^{\prime}$. Then $r_{1}$ has end $t_{4}$ in $G^{\prime} . C(1,2)$ is a circuit of $G$. But it cannot be the former, hence it is a $T^{\prime}$-join. Hence, $R_{3}$ has end $t_{2}$ in $G^{\prime}$ and $R_{2}$ has end $t_{3}$ in $G^{\prime}$. Hence, outcome (iv) occurs.

\subsubsection{Bounding the number of representations with anemones}

The key result in this section is the following,

Proposition 3.3.18. Let $M$ be a 3-connected p-cographic matroid. Then there exist anemone classes $\mathcal{F}_{1}, \ldots, F_{k}$ where $k \in O\left(\operatorname{rank}(M)^{3}\right)$ such that if $(G, T)$ is a $T_{4}$-representation of $M$ and there exists $\Phi$ that is a maximal ordinary anemone for $(G, T)$, then $(G, T) \in \mathcal{F}_{i}$ for some $i \in[k]$.

Before we proceed with the proof we wish to show that $k$ cannot be chosen as a constant in the previous result. Consider a wheel $W_{n}$ with vertices $v_{0}, v_{1}, \ldots, v_{n}$ and for $i=1, \ldots, n$, edges $v_{0} v_{i}$ and $v_{i} v_{i+1}$ (where $n+1=1$ ). Construct a graph $G$ from $W_{n}$ by applying the following construction for each $i \in[n]$ : (a) add to $W_{n}, 2$-connected graphs $G\left[P_{i}\right]$ and $G\left[P_{i}^{\prime}\right]$ where $\mathcal{B}_{G}\left(P_{i}\right)=\mathcal{B}_{G}\left(P_{i}^{\prime}\right)=\left\{v_{0}, v_{i}\right\}$, and (b) let $\Sigma$ such that $\Sigma \cap\left\{v_{i} v_{i+1} \mid i \in[n]\right\}=v_{1} v_{2}$ and such that for all $i \in[n], \Sigma \cap\left(P_{i} \cup P_{i}^{\prime}\right) \subseteq \delta_{G}\left(v_{0}\right)$ and there exists an odd circuit of $(G, \Sigma)$ contained in $P_{i}$ (resp. $P_{i}^{\prime}$ ) that is using $v_{0}$ and avoiding $v_{i}$. Then for every $i \in[n]$ we can resign $(G, \Sigma)$ to get a signature $\Gamma_{i} \subseteq \delta_{G}\left(v_{0}\right) \cup \delta_{G}\left(v_{i}\right)$. Denote by $\left(H_{i}, T_{i}\right)$ the graft obtained by unfolding $\left(G, \Gamma_{i}\right)$ on $v_{0}, v_{i}$. Then $\Phi=\left(\emptyset, G \backslash\left(P_{i} \cup P_{i}^{\prime}\right), P_{i}, P_{i}^{\prime}\right)$ is an ordinary anemone for $\left(H_{i}, T_{i}\right)$. Moreover, by Proposition 1.5.3 $\left(H_{i}, T_{i}\right)$ for all $i \in[n]$ are representations of the same even-cut matroid. We illustrate the construction in Figure 3.3,

Let $G$ be a connected graph and let $S \subseteq V(G)$. By a proper $S$-bridge we mean the connected graph obtained from a component of $G \backslash S$ by adding all the edges of $G$ that have one end in the component and one end in $S$. An $S$-bridge is either an edge with both ends in $S$ or a proper $S$-bridge. We have the following nice relation between proper bridges and petals of maximal flowers,

Remark 3.3.19. Let $\Phi=\left(E_{0},\left\{P_{1}, \ldots, P_{n}\right\}\right)$ be a maximal ordinary anemone for $(G, T)$. Then the petals $P_{1}, \ldots, P_{n}$ are exactly the proper $T$-bridges of $G$.

Remark 3.3.20. Let $\Phi=\left(E_{0},\left\{P_{1}, \ldots, P_{n}\right\}\right)$ be a maximal skewed anemone for $(G, T)$. Then the petals $P_{1}, \ldots, P_{n}$ are exactly the proper $\mathcal{B}_{G}\left(P_{1}\right)$-bridges of $G$. 

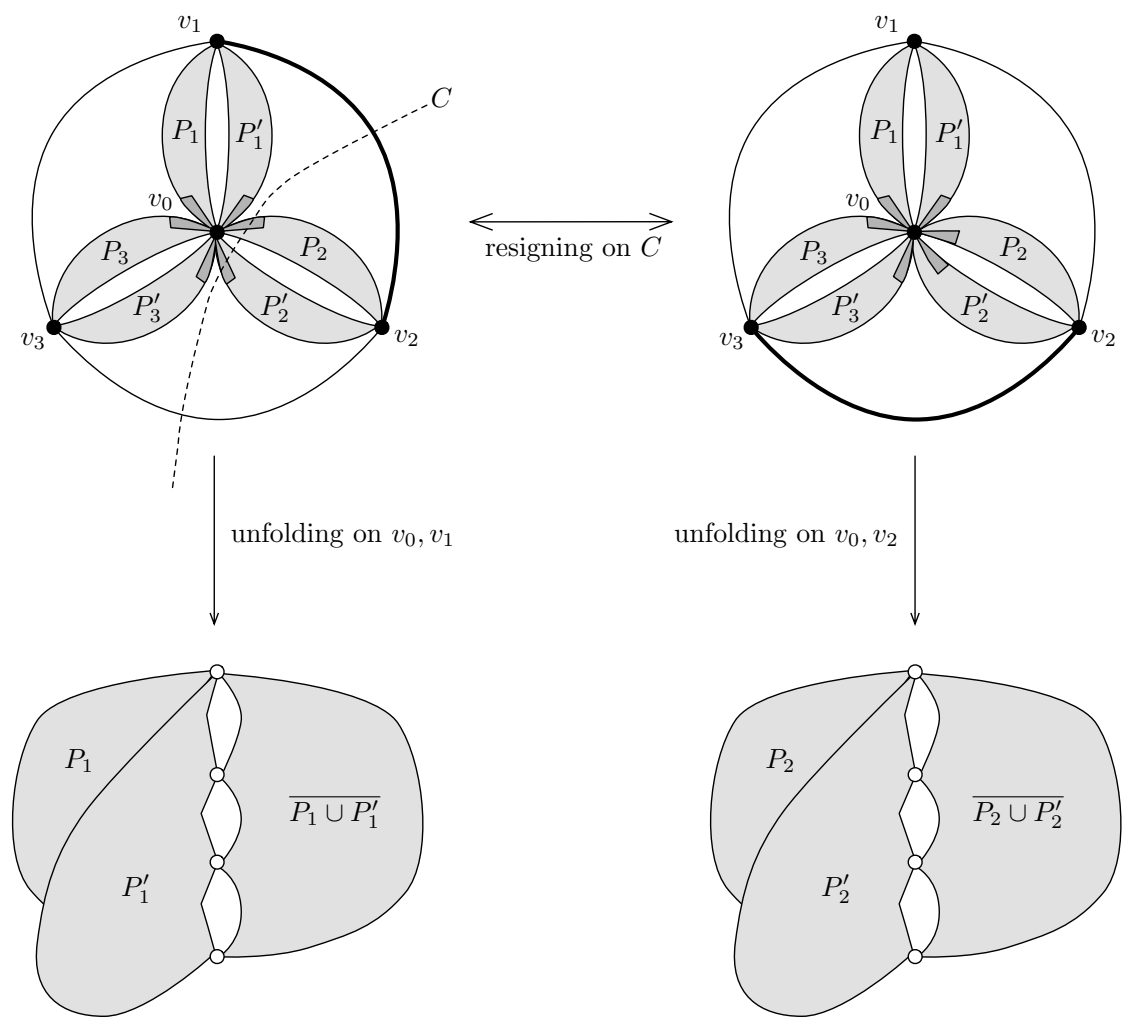

Figure 3.3: Linearly many ordinary anemones. Bold edges and shaded edges are odd. White vertices are terminal.

We are now ready for the proof of the main result in this section.

Proof of Theorem 3.3.18. Let $\left(G_{1}, T_{1}\right), \ldots,\left(G_{k}, T_{k}\right)$ be a maximal set of $T_{4}$-representations with the following properties:

- for each $i \in[k]$, there exists a maximal ordinary anemone $\Phi_{i}$ for $\left(G_{i}, T_{i}\right)$,

- for every distinct $i, j \in[k],\left(G_{i}, T_{i}\right)$ and $\left(G_{j}, T_{j}\right)$ are in distinct anemone equivalence classes.

We need to show that $k \in \mathcal{O}\left(|V|^{3}\right)$.

Claim 1. $\Phi_{1}, \ldots, \Phi_{k}$ are all distinct 
Proof. Suppose that for distinct $i, j \in[k], \Phi_{i}=\Phi_{j}$. Then $\Phi_{i}$ is a maximal ordinary anemone for both $\left(G_{i}, T_{i}\right)$ and $\left(G_{j}, T_{j}\right)$. By Proposition 3.3.17, $\left(G_{i}, T_{i}\right)$ and $\left(G_{j}, T_{j}\right)$ are related by rearranging petals and edges. Hence, $\left(G_{i}, T_{i}\right)$ and $\left(G_{j}, T_{j}\right)$ are in the same anemone class, a contradiction. $\diamond$

Let $i \in[k]$. By Proposition 3.3.4 $\Phi_{i}$ is either: (i) a maximal ordinary anemone of $\left(G_{1}, T_{1}\right)$ or (ii) a maximal skewed anemone of $\left(G_{1}, T_{1}\right)$. By Remark 3.3.19 it follows that there is a unique ordinary anemone as in (i). By Remark 3.3.20 it follows that there are at most $\left(\begin{array}{c}|V| \\ 3\end{array}\right)$ skewed anemones as in (ii) because it is the number of choices for the three-vertex boundary. It follows that $k \in \mathcal{O}\left(|V|^{3}\right)$ as required.

\subsubsection{Extending anemones}

Suppose that we have a p-cographic matroid $M$, and that $N$ is obtained by deleting or contracting a single element of $M$. The proof of Theorem 3.2.1 (as well as the membership algorithm for p-cographic matroids) relies on the fact that we can describe the representations of $M$ from the representations of $N$. In particular, we need to show that the number of equivalence classes and anemone classes of $N$ and $M$ only differ by a polynomial in $|E(N)|$. Two key steps are propositions 3.3.25 and 3.3.27 in this section.

First let us count the maximum number of grafts in an anemone class.

Remark 3.3.21. Let $\Phi$ be an anemone for some graft $(G, T)$. If $\Phi$ has $k$ petals, then the anemone class obtained generated by $\Phi$ and $(G, T)$ has cardinality at most $8 \times 3^{k}$.

Proof. There are at most $2^{3}$ ways of rearranging loose edges, and each petal can be rearranged in three possible ways (see Definition 3.3.2).

Before we proceed further we need some additional results and definitions.

Remark 3.3.22. Let $M$ be a matroid and $N=M \backslash$ e for some e $\in E(M)$. Then $M$ is determined by the cycles of $N$ as well as a unique cycle of $M$ using $e$.

Proof. Let $C$ be the unique cycle of $M$ using $e$. For any cycle $C_{1}$ of $M$ using $e, C_{2}=C \triangle C_{1}$ is a cycle of $M$ avoiding $e$. Thus, $C_{1}=C \triangle C_{2}$.

Proposition 3.3.23. Let $M$ be an p-cographic matroid and let eE(M). Let $N=M / e$ and suppose that both $M$ and $N$ are 3-connected. Consider a set $\Gamma \subseteq E(N)$ where $\Gamma \cup\{e\}$ is a cocycle of $M$. Suppose that $(G, T)$ is representation of $N$. Then $\left(G^{\prime}, T^{\prime}\right)$ is a representation of $M$ that extends $N$ if and only if $\left(G^{\prime}, T^{\prime}\right)$ satisfies one of the following, 
i. $\Gamma$ is a $\left\{v_{1}, v_{2}\right\}$-join and $G^{\prime}$ is obtained from $G$ by adding edge $e=\left(v_{1}, v_{2}\right)$, or

ii. For every $T$-join $J$ of $(G, T), \Gamma \triangle J$ is a $\left\{v_{1}, v_{2}\right\}$-join and $G^{\prime}$ is obtained from $G$ by adding edge $e=\left(v_{1}, v_{2}\right)$.

Proof. Suppose $\left(G^{\prime}, T^{\prime}\right)$ is a representation of $M$ that extends $N$. By Remark 1.4.4, $\left(G^{\prime}, T^{\prime}\right) \backslash e=$ $(G, T)$. Thus $T^{\prime}=T$. Since $\Gamma \cup\{e\}$ is a cocycle of $M, \Gamma \cup\{e\}$ is either a cycle of $G^{\prime}$ or a $T$-join of $\left(G^{\prime}, T\right)$. For the former case, $(i)$ holds. For the latter case, let $J$ be a $T$-join of $(G, T)$. Since $J$ is also a $T$-join of $\left(G^{\prime}, T\right),(\Gamma \cup\{e\}) \triangle J$ is a cycle of $G^{\prime}$. Thus, $(i i)$ holds. The converse follows from Remark 3.3.22 (by duality).

Proposition 3.3.24. Let $M$ be an p-cographic matroid and let $e \in E(M)$. Let $N=M \backslash e$ and suppose that both $M$ and $N$ are 3-connected. Consider a set $\Gamma \subseteq E(N)$ where $\Gamma \cup\{e\}$ is a cycle of $M$. Suppose that $(G, T)$ is representation of $N$. Then $\left(G^{\prime}, T^{\prime}\right)$ is a representation of $M$ that extends $(G, T)$ where $\left|T^{\prime}\right|=|T|$ if and only if $\left(G^{\prime}, T^{\prime}\right)$ can be constructed in the following way,

i. find $\Gamma^{\prime}=\Gamma \triangle D$ where $D$ is an even cut of $(G, T)$ and $\Gamma^{\prime} \subseteq \delta_{G}(v)$ for some $v \in V(G)$,

ii. $G^{\prime}$ is obtained from $G$ by splitting $v$ according to $\Gamma^{\prime}$ into $v_{1}, v_{2}$ and adding edge e $=\left(v_{1}, v_{2}\right)$,

iii. if $v \notin T$ then $T^{\prime}=T$,

iv. if $v \in T$ then $T^{\prime}=T-\{v\} \cup\left\{v_{3-i}\right\}$ for some $i \in[2]$ where $v_{i}$ is incident to the edges of $\Gamma^{\prime}$ in $G^{\prime}$.

Moreover, if e is not a loop of $M$, then $\Gamma^{\prime} \neq \emptyset$. If there is no parallel edge of e in $M$, then $\left|\Gamma^{\prime}\right|>1$ and $\left|\delta(v) \triangle \Gamma^{\prime}\right|>1$.

Proof. Suppose $\left(G^{\prime}, T^{\prime}\right)$ is a representation of $M$ that extends $N$. By Remark 1.4.4, $\left(G^{\prime}, T^{\prime}\right) / e=$ $(G, T)$. Let $e=\left(v_{1}, v_{2}\right)$ and let $v$ be a vertex obtained by contracting $e$. Since $\left|T^{\prime}\right|=|T|$, not all of $v_{1}, v_{2}$ are terminals. If both $v_{1}, v_{2}$ are not terminals, then $T^{\prime}=T$ and $v \notin T$. If exactly one of $v_{1}, v_{2}$ is a terminal, then $T^{\prime}=T-\{v\} \cup\left\{v_{i}\right\}$ for some $i \in[2]$ and $v \in T$. We may assume that $v_{1}$ is not a terminal. Then $D=\delta_{G^{\prime}}\left(v_{1}\right) \triangle(\Gamma \cup\{e\})$ is an even cut of $\left(G^{\prime}, T^{\prime}\right)$ avoiding $e$. Thus, $D$ is an even cut of $(G, T)$. Note that $v_{1}$ is incident to all edges of $\Gamma^{\prime}$ in $G^{\prime}$. The converse follows from Remark 3.3.22. The last part follows from Proposition 2.3.3.

Let $M$ be a p-cographic matroid and let $(G, T)$ be a $T_{4}$-representation. We say that $(G, T)$ is of anemone type if there exists $\Phi$ that is an anemone for $(G, T)$. 
Proposition 3.3.25. Let $M$ be a p-cographic matroid, let $e \in E(M)$ and let $N=M$ /e where $M$ and $N$ are 3-connected. Let $\left(G_{o}, T\right)$ be a $T_{4}$-representation of $N$ and let $\Phi=\left(E_{0},\left\{P_{1}, \ldots, P_{k}\right\}\right)$ be an ordinary anemone for $\left(G_{o}, T\right)$. Let $\mathcal{F}_{N}$ be the anemone class generated by $\Phi$ and $\left(G_{o}, T\right)$. Let $\mathcal{F}_{M}$ be the set of $T_{4}$-representations of $M$ that extend some representation in $\mathcal{F}_{N}$. Then either,

\section{i. $\mathcal{F}_{M}$ is contained in at most 216 equivalence classes, or}

ii. $\mathcal{F}_{M}$ is contained in a unique anemone class.

Moreover, suppose that we are given a set $\Gamma \subseteq E(N)$ where $\Gamma \cup\{e\}$ is a cocycle of $M$. Then together with $\left(G_{o}, T\right)$ we can construct in time polynomial in $|E(G)|$ the equivalence classes in (i) or the anemone class in (ii).

Proof. In this thesis, we will omit the proof for the algorithmic part. Let $\Gamma \subseteq E(N)$ where $\Gamma \cup\{e\}$ is a cocycle of $M$. For $i \in[k]$, let $H_{i}=G\left[P_{i}\right]$ and let $\Gamma_{i}=\Gamma \cap P_{i}$. Let $(G, T) \in \mathcal{F}_{N}$. Suppose that $(G, T)$ extends to some representation in $\mathcal{F}_{M}$. It follows from Proposition 3.3.23 that, after possibly replacing $\Gamma$ by $\Gamma \triangle J$ where $J$ is a $T$-join of $(G, T)$, we have $V_{G}(\Gamma)=\left\{v_{1}, v_{2}\right\}$ for some $\left\{v_{1}, v_{2}\right\} \subseteq V(G)$ and $e=\left(v_{1}, v_{2}\right)$. Consider first the case where $v_{1}, v_{2} \in V\left(H_{i}\right)$ for some $i \in[k]$. Then for an arbitrary $\left(G^{\prime}, T\right)$ in the anemone class $\mathcal{F}_{N},\left(G^{\prime}, T\right)$ is obtained from $(G, T)$ by rearranging petals and loose edges. It means that every representation in $\mathcal{F}_{N}$ extends to a representation of $M$. Moreover, it is easy to check that the resulting representations remain related by rearranging petals and edges. Hence, outcome $(i i)$ occurs.

Thus, we may assume that for distinct $i, j \in[k], v_{1} \in \mathcal{I}_{G}\left(P_{i}\right)$ and $v_{2} \in \mathcal{I}_{G}\left(P_{j}\right)$. Let $\left(G^{\prime}, T\right)$ be an arbitrary representation in $\mathcal{F}_{N}$.

Claim 1. $\left(G^{\prime}, T\right)$ extends to $M$ if and only if $P_{i}$ and $P_{j}$ are rearranged from $(G, T)$ in the same way.

Proof. Suppose that $P_{i}$ and $P_{j}$ are rearranged in a different way. Since $V_{G}(\Gamma)=\left\{v_{1}, v_{2}\right\}$, $V_{G^{\prime}}(\Gamma)=\left\{v_{1}, v_{2}, t_{1}, t_{2}\right\}$ for some distinct terminals $t_{1}, t_{2} \in T$. This contradicts Proposition 3.3.23.

It follows that all representations $(G, T) \in \mathcal{F}_{N}$ that extend to some representation of $M$ are exactly the representations for which $P_{i}$ and $P_{j}$ are rearranged like they are one petal. Moreover, either we have at least 4 petals, in which case outcome (ii) occurs, or there are at most 3 petals in which case outcome $(i)$ occurs as $8 \times 3^{3}=216$.

We leave the proof of the following remark as an exercise (it is equivalent to the problem of checking if a graph is bipartite). 
Remark 3.3.26. Let $(G, \Gamma)$ be a signed graph and let $R \subseteq V(G)$. Then we can check in time polynomial in $|E(G)|$ if there exists a cut $\delta(U)$ such that all edges in $\Gamma \triangle \delta(U)$ have at least one endpoint in $R$.

We say that an ordinary anemone $\Phi=\left(E_{0},\left\{P_{1}, \ldots, P_{k}\right\}\right)$ is degenerate if it is also a skewed anemone.

Proposition 3.3.27. Let $M$ be a p-cographic matroid, let $e \in E(M)$ and let $N=M \backslash$ e where $M$ and $N$ are 3-connected. Let $\left(G_{o}, T\right)$ be a $T_{4}$-representation of $N$ and let $\Phi=\left(E_{0},\left\{P_{1}, \ldots, P_{k}\right\}\right)$ be a maximal ordinary anemone for $\left(G_{o}, T\right)$. Let $\mathcal{F}_{N}$ be the anemone class generated by $\Phi$ and $\left(G_{o}, T\right)$. Let $\mathcal{F}_{M}$ be the set of $T_{4}$-representations of $M$ that extend some representation $(G, T)$ in $\mathcal{F}_{N}$ such that $\Phi$ is not degenerate in $(G, T)$. Then either,

i. $\mathcal{F}_{M}$ is contained in at most 216 equivalence classes, or

ii. $\mathcal{F}_{M}$ is contained in a unique anemone class.

Moreover, suppose that we are given a set $\Gamma \subseteq E(N)$ where $\Gamma \cup\{e\}$ is a cycle of $M$. Then together with $\left(G_{o}, T\right)$ we can construct in time polynomial in $|E(G)|$ the equivalence classes in (i) or find a representative for the anemone class in (ii).

Proof. In this thesis, we will omit the proof for the algorithmic part. Let $(G, T),\left(G^{\prime}, T\right) \in$ $\mathcal{F}_{N}$ such that $\Phi$ is non-degenerate ordinary anemone in $(G, T)$ and $\left(G^{\prime}, T\right)$, respectively. By Proposition 3.3.24, we may assume that there exist $v \in V(G)$ and $\Gamma \subseteq E(G)$ such that $\Gamma \subseteq \delta_{G}(v)$ and that $(G, T)$ extends by splitting $v$ according to $\Gamma$. Similarly, we may assume that there exists $v^{\prime} \in V\left(G^{\prime}\right)$ and $\Gamma^{\prime} \subseteq E\left(G^{\prime}\right)$ such that $\Gamma^{\prime} \subseteq \delta_{G^{\prime}}\left(v^{\prime}\right)$ and that $\left(G^{\prime}, T\right)$ extends by splitting $v^{\prime}$ according to $\Gamma^{\prime}$. Then, for some $U \subseteq V(G), \Gamma \triangle \Gamma^{\prime}=\delta_{G}(U)$. For $i \in[k]$, let $\Gamma_{i}=\Gamma \cap P_{i}$ and let $\Gamma_{i}^{\prime}=\Gamma^{\prime} \cap P_{i}$. Then, $\Gamma_{i} \triangle \Gamma_{i}^{\prime}=\delta_{G\left[P_{i}\right]}\left(U_{i}\right)$ for some $U_{i} \subseteq V\left(P_{i}\right)$.

Claim 1. If $\Gamma_{i} \neq \Gamma_{i}^{\prime}$, then there exists a vertex $u \in V_{G}\left(P_{i}\right)$ such that $\Gamma_{i}=\Gamma_{i}^{\prime} \triangle \delta_{G\left[P_{i}\right]}(u)$.

Proof. Since $\Gamma_{i} \neq \Gamma_{i}^{\prime}$, we may assume that $\Gamma_{i} \neq \emptyset$. Then, $v \in V_{G}\left(P_{i}\right)$. Suppose $\Gamma_{i}^{\prime}=\emptyset$. Since $\Gamma_{i} \triangle \Gamma_{i}^{\prime}$ is a cut of $G\left[P_{i}\right]$, so $\Gamma_{i}$ is a cut of $G\left[P_{i}\right]$. Thus, $\Gamma_{i}=\delta_{G\left[P_{i}\right]}(v)$. Thus, $\Gamma_{i}=\Gamma_{i}^{\prime} \triangle \delta_{G\left[P_{i}\right]}(v)$. Now, suppose $\Gamma_{i}^{\prime} \neq \emptyset$. Then there exists a vertex $w \in V_{G}\left(P_{i}\right)$ such that $\Gamma_{i}^{\prime} \subseteq \delta_{G\left[P_{i}\right]}(w)$. If $v=w$, then $\Gamma_{i} \triangle \Gamma_{i}^{\prime} \subseteq \delta_{G\left[P_{i}\right]}(v)$ is a cut of $V_{G}\left(P_{i}\right)$. Since both $\Gamma_{i}$ and $\Gamma_{i}^{\prime}$ are not empty, $\Gamma_{i}^{\prime}=$ $\Gamma_{i} \triangle \delta_{G\left[P_{i}\right]}(v)$. Thus, we may assume that $v \neq w$. Then $\{v, w\}$ forms a two-vertex cut-set of $G\left[P_{i}\right]$. Let $(X, \bar{X})$ be a 2-separation of $G\left[P_{i}\right]$. If $\mathcal{I}_{G\left[P_{i}\right]}(X) \cap T=\emptyset$, then $\lambda_{M}(X)=1$ by Proposition 2.3.3, contradicting 3-connectivity of $M$. Thus, $\mathcal{I}_{G\left[P_{i}\right]}(X) \cap T \neq \emptyset$, and similarly, $\mathcal{I}_{G\left[P_{i}\right]}(\bar{X}) \cap T \neq \emptyset$. Let $T=\left\{t_{1}, t_{2}, t_{3}, t_{4}\right\}$. There are following three cases up to exchanging $X$ and $\bar{X}$. 
Case 1. $\mathcal{B}_{G\left[P_{i}\right]}(X) \cap T=\emptyset$.

Without loss of generality, we may assume that $t_{1}, t_{2} \in \mathcal{I}_{G\left[P_{i}\right]}(X)$ and that $t_{3} \in \mathcal{I}_{G\left[P_{i}\right]}(\bar{X})$. Since $v, w \notin T, \Gamma=\Gamma_{i}$ and $\Gamma^{\prime}=\Gamma_{i}^{\prime}$. Since $\Gamma \triangle \Gamma^{\prime}$ is a cut of $G$, for every $j \neq i, \mathcal{B}_{G}\left(P_{j}\right)=$ $\left\{t_{1}, t_{2}, t_{4}\right\}$. Thus, $\Phi$ is a degenerate ordinary anemone in $(G, T)$, a contradiction.

Case 2. $\left|\mathcal{B}_{G\left[P_{i}\right]}(X) \cap T\right|=1$.

Without loss of generality, we may assume the $\mathcal{B}_{G\left[P_{i}\right]}(X) \cap T=\left\{t_{1}\right\}, t_{2} \in \mathcal{I}_{G\left[P_{i}\right]}(X)$ and $t_{3} \in \mathcal{I}_{G\left[P_{i}\right]}(\bar{X})$. Since $\Gamma \triangle \Gamma^{\prime}$ is a cut of $G$, either for every $j \neq i, \mathcal{B}_{G}\left(P_{j}\right)=\left\{t_{1}, t_{2}, t_{4}\right\}$ or for every $j \neq i, \mathcal{B}_{G}\left(P_{j}\right)=\left\{t_{1}, t_{3}, t_{4}\right\}$. In both cases, $\Phi$ is a degenerate ordinary anemone in $(G, T)$, a contradiction.

Case 3. $\left|\mathcal{B}_{G\left[P_{i}\right]}(X) \cap T\right|=2$. Without loss of generality, we may assume the $\mathcal{B}_{G\left[P_{i}\right]}(X) \cap T=$ $\left\{t_{1}, t_{2}\right\}, t_{3} \in \mathcal{I}_{G\left[P_{i}\right]}(X)$ and $t_{4} \in \mathcal{I}_{G\left[P_{i}\right]}(\bar{X})$. Then, $X$ and $\bar{X}$ are both petals, contradicting maximality of $\Phi$.

Suppose $v \notin T$. Let $P_{i}$ be the petal such that $v \in \mathcal{I}_{G}\left(P_{i}\right)$. By 3-connectivity of $M$ and Proposition 3.3.24, $|\Gamma|>1$ and $|\delta(v) \triangle \Gamma|>1$. By Claim $1, v=v^{\prime}$ and either $\Gamma=\Gamma^{\prime}$ or $\Gamma=\Gamma^{\prime} \triangle \delta_{G}(v)$, in which case outcome (ii) occurs. Now suppose $v \in T$. Note that $D:=\Gamma \triangle \Gamma^{\prime}$ is an even cut of $(G, T)$ and every even cut of $(G, T)$ can be generated by cuts of the form $\delta_{G}\left(\mathcal{I}_{G}\left(P_{i}\right)\right)$ and a cuts of the form $\delta\left(t_{a}\right) \triangle \delta\left(t_{b}\right)$ where $a, b$ are distinct and $a, b \in$ [4]. By Claim 1 , for $i \in[k], D \cap P_{i}$ is either the empty set or is of the form $\delta_{G\left[P_{i}\right]}\left(t_{j}\right)$ for some $t_{j} \in T$. Thus, $D \cap P_{i}=\emptyset$ for all $i \in[k]$. Let $I$ be the set of all $i \in[k]$ such that $\Gamma_{i} \neq \emptyset$. Then, either $|[k]-I| \leq 4$, in which case outcome (i) occurs, or $|[k]-I| \geq 5$, in which case outcome (ii) occurs.

\subsection{Bounding the number of representations}

\subsubsection{Equivalence classes and anemone classes}

In this section, we will prove Theorem 3.2.1, which we restate here:

Theorem 3.2.1. Let $M$ be a 3-connected p-cographic matroid that is not cographic. Then the set of all $T_{4}$-representations of $M$ is contained in the union of a polynomial number of equivalence classes and a polynomial number of anemone classes. 
Let $M$ be a 3-connected p-cographic matroid that is not cographic. For a equivalence class $\mathcal{F}$ of $M$, we say that $\mathcal{F}$ is anemone type if for every $T_{4}$-representation $(G, T) \in \mathcal{F},(G, T)$ has a non-degenerate ordinary anemone. The Proposition 3.3.18 shows that the number of anemone classes for $M$ is bounded by a polynomial function of $E(M)$. Thus, it suffices to bound the number of equivalence classes that are not anemone types by a polynomial function of $|E(M)|$. First, in Section 3.4.2, we will prove Lemma 3.4.1 that there are polynomially many equivalence classes containing a representation with a degenerate ordinary anemone.

Lemma 3.4.1. Let $M$ be a 3-connected p-cographic matroid that is not cographic. Then the number of equivalence classes for $M$ that contains a representation with a degenerate ordinary anemone is bounded by polynomial function of $|E(M)|$.

Fortunately, by Proposition 2.2.1, column extension of an equivalence class does not increase the number of equivalence classes. In fact, if we have possible anemone classes after column extension, then we may reduce the number of equivalence classes. However, by Proposition 2.2.2, row extension of equivalence classes may double the number of equivalence classes so that eventually, we have exponentially many equivalence classes as we saw in Section 1.3.3. Let $\mathcal{F}$ be a equivalence class of $N$ and let $M$ be a 3-connected p-cographic row major of $N$. We say that $\mathcal{F}$ is row stable for $M$, if the set of all extensions of $\mathcal{F}$ to $M$ is contained in at most one equivalence class. Otherwise, we say that $\mathcal{F}$ is row unstable for $M$. In the Section 3.4.5, we will prove the following lemma stating that the number of row unstable equivalence classes $\mathcal{F}$ such that every $T_{4}$-representation in $\mathcal{F}$ has no ordinary anemone, is bounded by polynomial function of $|E(M)|$. Thus, row extension only increase the number of equivalence classes that are not anemone type by a polynomial function of $|E(M)|$.

Lemma 3.4.2. Let $N$ be a 3-connected p-cographic matroid that is not cographic. Let $M$ be a 3-connected p-cographic major of $N$. Let $\mathcal{F}_{1}, \ldots, \mathcal{F}_{k}$ be equivalence classes for $N$ that are row unstable for $M$. Suppose that every $T_{4}$-representation in $\mathcal{F}_{1} \cup \cdots \cup \mathcal{F}_{k}$ has no ordinary anemone. Then $k=O\left(\left|E(M)^{14}\right|\right)$.

Proof of Theorem 3.2.1. Let $N$ be a minimally non-cographic minor of $M$. Note that $N$ is 3connected and p-cographic. By Seymour [13], there exists a sequence of 3-connected matroids $N_{1}, \ldots, N_{k}$, where $N=N_{1}, M=N_{k}$ and $N_{i}$ is a column or row minor of $N_{i+1}$ for all $i \in[k-1]$. Since $N$ is non-cographic and $M$ is p-cographic, $N_{i}$ is p-cographic and non-cographic for all $i \in[k]$. By Theorem 1.1.1, $N$ has a constant size. Thus, the set of all $T_{4}$-representations of $M$ is contained in a constant number equivalence classes. By the Proposition 3.3.18, there is a polynomial function $p_{1}$ such that for each $i \in[k]$, the number of anemone classes for $N_{i}$ is bounded by $p_{1}(|E(M)|)$. Thus, it suffices to prove that there exists a polynomial function $p_{2}$ 
such that for $i \in[k-1]$ the number of equivalence classes that are not anemone type from $N_{i}$ to $N_{i+1}$ is increased by $p_{2}(|E(M)|)$. Note that $N=N_{1}$ has the constant size, so there is a bounded number of equivalence classes for $N$.

Consider the case that $N_{i}$ is a column minor of $N_{i+1}$. By Proposition 2.2.1, an extension from each equivalence class for $N_{i}$ does not increase the number of equivalence classes for $N_{i+1}$. By Proposition 3.3.25, an extension from each anemone classes for $N_{i}$ increases the number of equivalence classes for $N_{i+1}$ by at most 216. By Proposition 3.3.18, there are at most $p_{1}(|E(M)|)$ anemone classes.

Now consider the case that $N_{i}$ is a row minor of $N_{i+1}$. By Proposition 2.2.2, an extension from each equivalence class for $N_{i}$ increases the number of equivalence classes for $N_{i+1}$ by at most 1. By Lemma 3.4.1 and Lemma 3.4.2, there exists a polynomial function $p_{3}$ such that the number of such extensions is bounded by $p_{3}(|E(M)|)$. By Proposition 3.3.27, an extension from each anemone classes for $N_{i}$ increases the number of equivalence classes for $N_{i+1}$ by at most 216 . By Proposition 3.3.18, there are at most $p_{1}(|E(M)|)$ anemone classes. Thus, $p_{2}=216 \times p_{1}+p_{3}$ is a polynomial function that we want.

\subsubsection{Equivalence classes that are not anemone type}

Let $M$ be a 3-connected p-cographic matroid that is not cographic. Let $(H, T)$ be a $T_{4}$-representation for $M$ that contains a non-degenerate ordinary anemone $\Phi=\left(E_{0},\left\{P_{1}, \ldots, P_{k}\right\}\right)$. Note that there exists three distinct petals $P_{i}, P_{j}, P_{l}$ for $i, j, l \in[k]$ such that

(1) $\mathcal{B}_{H}\left(P_{i}\right)=T$, and

(2) $\mathcal{B}_{H}\left(P_{j}\right) \cup \mathcal{B}_{H}\left(P_{l}\right)=T$.

The following lemma implies that equivalence classes which contain a representation with a non-degenerate ordinary anemone are anemone type.

Lemma 3.4.3. Let $M$ be a 3-connected p-cographic matroid that is not cographic. Let $(H, T)$ be a $T_{4}$-representation for $M$ that contains a non-degenerate ordinary anemone $\Phi=\left(E_{0},\left\{P_{1}\right.\right.$, $\left.\left.\ldots, P_{k}\right\}\right)$. Suppose $\mathcal{F}$ is an equivalence class containing $(H, T)$. Then $\mathcal{F}=\{(H, T)\}$.

Proof. Let $T=\left\{t_{1}, t_{2}, t_{1}, t_{2}\right\}$ and let $(X, \bar{X})$ be a 2-separation of $H$ where $\mathcal{B}_{H}(X)=\{u, v\}$. If $X \subseteq P_{i}$ for some $i \in[k]$, then by Proposition 2.3.3, $\lambda_{M}(X)=1$ contradicting 3-connectivity of $M$. Thus, $X$ and $P_{i}$ are crossing for some $i \in[k]$. We may assume that $u \in \mathcal{I}_{H}\left(P_{i}\right)$ and $v \in \mathcal{I}_{H}\left(\bar{P}_{i}\right)$. Let $N=\operatorname{cycle}(H)$. Then $\lambda_{N}(X)=1$ and $\lambda_{N}\left(P_{i}\right) \in\{2,3\}$. By submodularity 
of connectivity function, $\lambda_{N}\left(X \cap P_{i}\right)+\lambda_{N}\left(X \cup P_{i}\right) \leq \lambda_{N}(X)+\lambda_{N}\left(P_{i}\right) \leq 4$. Suppose that $\lambda_{N}\left(X \cap P_{i}\right)=\lambda_{N}\left(X \cup P_{i}\right)=2$. Then, $\left|\mathcal{B}_{H}\left(X \cap P_{i}\right) \cap \mathcal{B}_{H}\left(X \cap \bar{P}_{i}\right)\right|=2$. and $\mid \mathcal{B}_{H}(\bar{X} \cap$ $\left.P_{i}\right) \cap \mathcal{B}_{H}\left(\bar{X} \cap \bar{P}_{i}\right) \mid=2$. Since for $j \in[k]-\{i\}, H\left[P_{j}\right] \backslash \mathcal{B}_{H}\left(P_{j}\right)$ is connected, $v \in \mathcal{I}_{H}\left(P_{j}\right)$ contradicting that every pair of petals can have a common vertex only in $T$. Thus, we may assume $\lambda_{N}\left(X \cap P_{i}\right)=1$. By the similar argument, $\lambda_{N}\left(\bar{X} \cap P_{i}\right)=1$ or $\lambda_{N}\left(X \cap \bar{P}_{i}\right)=1$. If $\lambda_{N}\left(\bar{X} \cap P_{i}\right)=1$, then $\left|\mathcal{B}_{H}\left(P_{i}\right)\right|=2$, giving a contradiction. Thus, $\lambda_{N}\left(X \cap \bar{P}_{i}\right)=1$. Since $M$ is 3-connected, $X \cap P_{i}$ and $X \cap \bar{P}_{i}$ share a vertex (say $t_{1}$ ) in $T$. We may assume that $X \cap \bar{P}_{i}$ is an edge of $P_{j}$ where $j \in[k]$ and $i \neq j$. Then for $l \in[k]-\{i, j\}, \mathcal{B}_{H}\left(P_{l}\right)=\left\{t_{2}, t_{3}, t_{4}\right\}$. Thus, $X$ is the unique 2-separation of $H$. However, Whitney-flip on $X$ increases the number of terminal vertices to size of 6 , giving a contradiction.

Proof of Theorem 3.4.1. Let $\mathcal{F}$ be a equivalence class that contains a $T_{4}$-representation with a degenerate ordinary anemone $\Phi$. By Lemma 3.4.3, for every $(H, T) \in \mathcal{F}, \Phi$ is not a nondegenerate ordinary anemone in $(H, T)$. Thus, $\Phi$ has a petal $P_{i}$ where $\left|\mathcal{B}_{H}\left(P_{i}\right)\right|=4$ and there exists $t_{1}, t_{2}, t_{3} \in T$ such that for a petal $P_{j} \neq P_{i}$ of $\Phi, \mathcal{B}_{H}\left(P_{j}\right)=\left\{t_{1}, t_{2}, t_{3}\right\}$. Thus, for each anemone class $\mathcal{F}^{\prime}$, there are at most 4 representations that has a degenerate ordinary anemone. By Proposition 3.3.18, there exists a polynomial function $p$ such that the number of anemone classes for $M$ is bounded by $p(|E(M)|)$. Thus, the number of equivalence classes that contains a $T_{4}$-representation with a degenerate ordinary anemone is bounded by $4 \times p(|E(M)|)$.

\subsubsection{Homologous representations}

In [6], the following lemma explains how row unstable equivalence classes extend.

Lemma 3.4.4. Let $M$ be an even cut matroid that is not cographic and let $\mathcal{F}$ be an equivalence class of $M$. Let $N$ be a row major of $M$ with no loops or coloops. Suppose that the set $\mathcal{F}^{\prime}$ of extensions of $\mathcal{F}$ to $N$ is the union of two equivalence classes $\mathcal{F}_{1}$ and $\mathcal{F}_{2}$. Then for any $\left(H_{1}, T_{1}\right) \in$ $\mathcal{F}_{1}$ and $\left(H_{2}, T_{2}\right) \in \mathcal{F}_{2},\left(H_{1}, T_{1}\right)$ and $\left(H_{2}, T_{2}\right)$ are basic siblings or nested siblings.

A basic sibling and a nested sibling are defined in [11]. As corollary, if $M$ and $N$ are pcographic, then we have the following result from [5].

Corollary 3.4.5. Let $M$ be a 3-connected p-cographic matroid that is not cographic. Let $\mathcal{F}$ be a row unstable equivalence class of $M$. Then there exists a representation $(H, T)$ in $\mathcal{F}$ and a partition $P=\left(\left\{u_{1}, v_{1}\right\},\left\{u_{2}, v_{2}\right\}\right)$ of $T$, such that, for $i \in\{1,2\}$, a representation obtained by adding an edge $\left(u_{i}, v_{i}\right)$ to $(H, T)$ is contained in $\mathcal{F}_{i}$. 
In this case, we call $(H, T)$ a representative of $\mathcal{F}$ with a pairing $P$ and we call $(H, T, P)$ an extended $T_{4}$-representation for $M$.

For $i=1,2$, let $\left(H_{i}, T_{i}\right)$ be a representative of a row unstable equivalence class $\mathcal{F}_{i}$ with a pairing $P_{i}$ and let $\left(G_{i}, \Sigma_{i}\right)$ be a signed graph obtained by folding $\left(H_{i}, T_{i}\right)$ according to $P_{i}$. Then we say that $\left(H_{1}, T_{1}, P_{1}\right)$ and $\left(H_{2}, T_{2}, P_{2}\right)$ are homologous if $\left(G_{1}, \Sigma_{1}\right)$ and $\left(G_{2}, \Sigma_{2}\right)$ are equivalent. The following lemma explains the relation between row unstable equivalence classes.

Lemma 3.4.6. Let $N$ be a 3-connected p-cographic matroid which is not cographic and let $\mathcal{F}_{1}, \ldots, \mathcal{F}_{k}$ be equivalence classes for $N$ that is row unstable for a row major $M$ of $N$. For $i \in[k]$, Let $\left(H_{i}, T_{i}\right)$ be a representative of $\mathcal{F}_{i}$ with a pairing $P_{i}$. Then $\left(H_{1}, T_{1}, P_{1}\right), \cdots,\left(H_{k}, T_{k}\right.$, $\left.P_{k}\right)$ are homologous.

Proof. Let $e \in E(M)$ where $N=M / e$. For $i \in\{1,2\}$, let $\left(H_{i}^{\prime}, T_{i}^{\prime}\right)=\left(H_{i}, T_{i}\right) / e$. By Corollary 3.4.5, ends of $e$ are vertices of $T_{1}$, so $\left|T_{1}^{\prime}\right|=2$. Thus, $G_{1}$ can be obtained by identifying two vertices of $\left|T_{1}^{\prime}\right|$ from $\left(H_{1}^{\prime}, T_{1}^{\prime}\right)$ and $\operatorname{cut}\left(G_{1}\right)=\operatorname{ecut}\left(H_{1}^{\prime}, T_{1}^{\prime}\right)$. Similarly, $\operatorname{cut}\left(G_{2}\right)=\operatorname{ecut}\left(H_{2}^{\prime}, T_{2}^{\prime}\right)$. Since $\operatorname{ecut}\left(H_{1}, T_{1}\right)=\operatorname{ecut}\left(H_{2}, T_{2}\right), \operatorname{ecut}\left(H_{1}^{\prime}, T_{1}^{\prime}\right)=\operatorname{ecut}\left(H_{2}^{\prime}, T_{2}^{\prime}\right)$. Thus, $\operatorname{cut}\left(G_{1}\right)=\operatorname{cut}\left(G_{2}\right)$. Thus, $G_{1}$ and $G_{2}$ are equivalent, and $\left(G_{1}, \Sigma_{1}\right),\left(G_{2}, \Sigma_{2}\right)$ are also equivalent.

\subsubsection{Whitney flip sequences and the gap function}

Let $\left(H_{1}, T_{1}, P_{1}\right)$ and $\left(H_{2}, T_{2}, P_{2}\right)$ be homologous extended $T_{4}$-representations for $M$. For $i=$ 1,2 , let $\left(G_{i}, \Sigma_{i}\right)$ be a signed-graph obtained by folding $\left(H_{i}, T_{i}\right)$ according to $P_{i}$ and let $\left\{u_{i}, v_{i}\right\}$ be blocking pair obtained by identifying vertices of $T_{i}$. Then, $\Sigma_{i} \subseteq \delta\left(u_{i}\right) \cup \delta\left(v_{i}\right) .\left(G_{i}, \Sigma_{i},\left\{u_{i}, v_{i}\right\}\right)$ is an extended BP-representation for $M$ if $\left(G_{i}, \Sigma_{i}\right)$ is a BP-representation for $M$ and $\Sigma_{i} \subseteq$ $\delta\left(u_{i}\right) \cup \delta\left(v_{i}\right)$. In this case, $R_{i}=\left(G_{i}, \Sigma_{i},\left\{u_{i}, v_{i}\right\}\right)$ is obtained by folding $\left(H_{i}, T_{i}, P_{i}\right)$. We say that $R_{1}, R_{2}$ are equivalent if $\left(G_{1}, \Sigma_{1}\right),\left(G_{2}, \Sigma_{2}\right)$ are equivalent. Since $R_{1}, R_{2}$ are equivalent, we can find a sequence of Whitney-flips from $G_{1}$ to $G_{2}$.

Let $G_{1}, \ldots, G_{k+1}$ be graphs and let $X_{1}, \ldots, X_{k}$ be a 2 -separation of $G_{1}, \ldots, G_{k}$, respectively. Suppose that for $i=1, \ldots, k, G_{i+1}$ is a graph obtained by performing a Whitney-flip on $X_{i}$ in $G_{i}$. Then, we say that $\mathcal{S}=\left(X_{1}, \ldots, X_{k}\right)$ is a $w$-sequence of $G_{1}$ and denote $G_{k+1}=W_{f l i p}[G, \mathcal{S}]$. For $X, Y \in \mathcal{S}, X, Y$ are crossing if none of $X \cap Y, X \cap \bar{Y}, \bar{X} \cap Y, \bar{X} \cap Y$ is an empty set. Otherwise, $X, Y$ are non-crossing. We say that $\mathcal{S}$ is non-crossing if for every pairs $X, Y \in \mathcal{S}$, $X, Y$ are non-crossing. Note that if $\mathcal{S}$ is non-crossing, then we can rearrange the order of $\mathcal{S}$ arbitrarily. 
Let $\left(G_{1}, \Sigma_{1},\left\{u_{1}, v_{1}\right\}\right)$ and $\left(G_{2}, \Sigma_{2},\left\{u_{2}, v_{2}\right\}\right)$ be extended BP-representations for a matroid $M$. We say that $\left(\mathcal{S}_{1}, \mathcal{S}_{2}, \mathcal{S}_{3}\right)$ is a nice Whitney-flip sequence from $\left(G_{1}, \Sigma_{1},\left\{u_{1}, v_{1}\right\}\right)$ to $\left(G_{2}, \Sigma_{2}\right.$, $\left.\left\{u_{2}, v_{2}\right\}\right)$ if $\mathcal{S}_{2}$ is non-crossing w-sequence and there exist graphs $H_{1}$ and $H_{2}$, such that,

(1) for all $X \in \mathcal{S}_{1} \cup \mathcal{S}_{2} \cup \mathcal{S}_{3}, X$ is a minimal 2-separation(that is, $X$ induces connected graph after deleting boundary vertices of $X$ ).

(2) $H_{1}=W_{\text {flip }}\left[G_{1}, \mathcal{S}_{1}\right]$, where $\left\{u_{1}, v_{1}\right\} \cap \mathcal{B}_{G_{1}}(X)=\emptyset$ for all $X \in \mathcal{S}_{1}$,

(3) $H_{2}=W_{f l i p}\left[G_{2}, \mathcal{S}_{3}\right]$, where $\left\{u_{2}, v_{2}\right\} \cap \mathcal{B}_{G_{2}}(X)=\emptyset$ for all $X \in \mathcal{S}_{3}$, and

(4) $H_{2}=W_{\text {flip }}\left[H_{1}, \mathcal{S}_{2}\right]$, where $\left\{u_{1}, v_{1}\right\} \cap \mathcal{B}_{H_{1}}(X) \neq \emptyset$ and $\left\{u_{2}, v_{2}\right\} \cap \mathcal{B}_{H_{2}}(X) \neq \emptyset$ for all $X \in \mathcal{S}_{2}$.

By using the following lemma from [11], there exists a nice w-sequence $\left(\mathcal{S}_{1}, \mathcal{S}_{2}, \mathcal{S}_{3}\right)$ from $\left(G_{1}\right.$, $\left.\Sigma_{1},\left\{u_{1}, v_{1}\right\}\right)$ to $\left(G_{2}, \Sigma_{2},\left\{u_{2}, v_{2}\right\}\right)$.

Lemma 3.4.7. Let $G_{1}, G_{2}$ be 2-connected equivalent graphs and let $Z \subseteq V\left(G_{1}\right)$, where $|Z| \leq 2$. Then, there exist a w-sequence $\mathcal{S}_{1}$ of $G_{1}$ and a graph $H$ with a non-crossing $w$-sequence $\mathcal{S}_{2}$ such that:

(1) $H=W_{\text {flip }}\left[G_{1}, \mathcal{S}_{1}\right]$, where $Z \cup \mathcal{B}_{G_{1}}(X)=\emptyset$ for all $X \in \mathcal{S}_{1}$ and

(2) $G_{2}=W_{\text {flip }}\left[H, \mathcal{S}_{2}\right]$, where $Z \cup \mathcal{B}_{G_{1}}(X) \neq \emptyset$ for all $X \in \mathcal{S}_{2}$.

To use this lemma, we set $Z=\left\{u_{1}, v_{1}\right\}$. We may assume that each Whitney-flip in $\mathcal{S}_{1} \cup \mathcal{S}_{2}$ is minimal. Let $\mathcal{S}_{2}^{\prime}$ be a set of all $X \in \mathcal{S}_{2}$ such that $\left\{u_{2}, v_{2}\right\} \cap \mathcal{B}_{G_{2}}(X) \neq \emptyset$ and let $\mathcal{S}_{3}=\mathcal{S}_{2}-\mathcal{S}_{2}^{\prime}$. We can rearrange $\mathcal{S}_{2}$ into $\left(\mathcal{S}_{2}^{\prime}, \mathcal{S}_{4}\right)$, because $\mathcal{S}_{2}$ is non-crossing. Then $\left(\mathcal{S}_{1}, \mathcal{S}_{2}^{\prime}, \mathcal{S}_{3}\right)$ is a nice wsequence.

Let $R_{1}=\left(G_{1}, \Sigma_{1},\left\{u_{1}, v_{1}\right\}\right), R_{2}=\left(G_{2}, \Sigma_{2},\left\{u_{2}, v_{2}\right\}\right)$ be extended BP-representations for a matroid $M$. By using a nice w-sequence, we can define a function $\operatorname{gap}\left(R_{1}, R_{2}\right)$ by the minimum $\left|\mathcal{S}_{2}\right|$ among all nice w-sequences $\left(\mathcal{S}_{1}, \mathcal{S}_{2}, \mathcal{S}_{3}\right)$ from $R_{1}$ to $R_{2}$.

\subsubsection{The proof of Lemma 3.4.2}

\section{Extended BP-representations with large gap}

In this section, we will prove following Lemma 3.4.8 stating that if we have two homologous extended $T_{4}$-representations for a matroid with large gap, then at least one of them has an ordinary anemone. 
Lemma 3.4.8. Let $M$ be a 3-connected p-cographic matroid which is not cographic. Let $\left(H_{1}, T_{1}\right.$, $\left.P_{1}\right),\left(H_{2}, T_{2}, P_{2}\right)$ are homologous extended $T_{4}$-representation for $M$. For $i=1,2$, let $R_{i}=\left(G_{i}\right.$, $\left.\Sigma_{i},\left\{u_{i}, v_{i}\right\}\right)$ be an extended BP-representation obtained by folding $\left(H_{i}, T_{i}, P_{i}\right)$. If gap $\left(R_{1}, R_{2}\right)>$ 10 , then at least one of $\left(H_{1}, T_{1}\right)$ and $\left(H_{2}, T_{2}\right)$ has an ordinary anemone.

A w-sequence $\mathcal{S}=\left(X_{1}, \ldots, X_{k}\right)$ of a graph $G$ is a $w$-star of $G$ with a center $z$ if $\mathcal{S}$ is noncrossing and there exist distinct $z, v_{1}, \ldots, v_{k} \in V(G)$ such that for $i \in[k], \mathcal{B}_{G}\left(X_{i}\right)=\left\{z, v_{i}\right\}$. To prove Lemma 3.4.8, we need following Lemma 3.4.9 from [11] and Remark 3.4.10. We omit the proof of Remark 3.4.10.

Lemma 3.4.9. Let $G_{1}, G_{2}$ be equivalent graphs with $G_{2}=W_{\text {flip }}\left[G_{1}, \mathcal{S}\right]$ for some non-crossing w-sequence $\mathcal{S}$. Suppose that there exist vertices $z_{1} \in V\left(G_{1}\right)$ and $z_{2} \in V\left(G_{2}\right)$ such that $z_{1} \in$ $\mathcal{B}_{G_{1}}(X)$ and $z_{2} \in \mathcal{B}_{G_{2}}(X)$ for every $X \in \mathcal{S}$. Then $G_{2}=W_{\text {flip }}\left[G_{1}, \mathcal{S}^{\prime}\right]$ for some $\mathcal{S}^{\prime}$ which is a $w$-star of $G_{1}$ with center $z_{1}$ and a w-star of $G_{2}$ with center $z_{2}$.

Remark 3.4.10. Let $X_{1}, X_{2}, X_{3}$ be non-crossing 2-separations of a graph $G$. Suppose $z \in$ $\mathcal{B}_{G}\left(X_{1}\right) \cap \mathcal{B}_{G}\left(X_{2}\right)$ and $z \notin \mathcal{B}_{G}\left(X_{3}\right)$. Then, $X_{3} \cap\left(X_{1} \cup X_{2}\right)=\emptyset$ or $\bar{X}_{3} \cap\left(X_{1} \cup X_{2}\right)=\emptyset$.

Now, we need to define some terminology for signed graphs. Let $G$ be a connected graph and let $u, v$ be distinct vertices of $G$. Let $E_{0}$ be a set of edges of $G$ that have both ends in $\{u, v\}$ and let $P_{1}, \ldots, P_{k}$ be a partition of $E(G)-E_{0}$. We say that $\Phi=\left(E_{0},\left\{P_{1}, \ldots, P_{k}\right\}\right)$ where $k \geq 3$ is a graphic anemone of $G$ with a spine $\{u, v\}$, if

(1) for each $i \in[n], \mathcal{B}_{G}\left(P_{i}\right)=\{u, v\}$,

(2) for each $i \in[n], \mathcal{I}_{G}\left(P_{i}\right) \neq \emptyset$, and

(3) for each $i \in[n], G\left[P_{i}\right] \backslash\{u, v\}$ is connected.

The sets $P_{1}, P_{2}, \ldots, P_{n}$ are called the petals of $\Phi$.

Lemma 3.4.11. Let $M$ be a 3-connected p-cographic matroid that is not cographic. Let (H, $T, P)$ be an extended $T_{4}$-representation for $M$ and let $(G, \Sigma,\{u, v\})$ be an extended BPrepresentation obtained by folding $(H, T, P)$. Suppose that $\Phi=\left(E_{0},\left\{P_{1}, \ldots, P_{k}\right\}\right)$ is a graphic anemone of $G$ with a spine $\{u, v\}$. Then $\Phi$ is an ordinary anemone of $(H, T)$.

Proof. Since $(H, T)$ is a graft obtained by unfolding $(G, \Sigma),|T|=4$. Suppose that we split $u$ into $u_{1}, u_{2}$ and split $v$ into $v_{1}, v_{2}$ in $H$. Then, for every edge $e \in E_{0}$, the ends of $e$ are in $\left\{u_{1}, u_{2}, v_{1}, v_{2}\right\}$. Since $G\left[P_{i}\right] \backslash\{u, v\}$ is connected and $M$ is 3-connected, $H\left[P_{i}\right]$ is connected. Since $\mathcal{B}_{G}\left(P_{i}\right)=\{u, v\}, \mathcal{B}_{H}\left(P_{i}\right) \subseteq T$. By 3-connectivity of $M,\left|\mathcal{B}_{H}\left(P_{i}\right)\right| \geq 3$. 
We now prove Lemma 3.4.8.

Proof of Lemma 3.4.8. Suppose $\left(H_{1}, T_{1}\right)$ and $\left(H_{2}, T_{2}\right)$ have no ordinary anemone. Let $\left(\mathcal{S}_{1}, \mathcal{S}_{2}\right.$, $\mathcal{S}_{3}$ ) be a nice w-sequence from $R_{1}$ to $R_{2}$. Let $G_{1}^{\prime}=W_{f l i p}\left[G_{1}, \mathcal{S}_{1}\right]$ and Let $G_{2}^{\prime}=W_{f l i p}\left[G_{2}, \mathcal{S}_{2}\right]$. Note that for every 2-separation $X \in \mathcal{S}_{2}, \mathcal{B}_{G_{1}^{\prime}}(X) \cap\left\{u_{1}, v_{1}\right\} \neq \emptyset$ and $\mathcal{B}_{G_{2}^{\prime}}(X) \cap\left\{u_{2}, v_{2}\right\} \neq \emptyset$. Since, $\left(H_{1}, T_{1}\right)$ and $\left(H_{2}, T_{2}\right)$ have no ordinary anemone, there are at most two 2-separations in $\mathcal{S}_{2}$ that have both $u_{1}, v_{1}$ as its boundary vertices in $G_{1}^{\prime}$. Thus, we may assume there is no such 2-separations and $\left|\mathcal{S}_{2}\right| \geq 11$. Similarly, we may assume there is no 2-separations in $\mathcal{S}_{2}$ that have both $u_{2}, v_{2}$ as its boundary vertices in $G_{2}^{\prime}$ and $\left|\mathcal{S}_{2}\right| \geq 13$. Let $\left(X_{u} u, X_{u} v, X_{v} u, X_{v} v\right)$ be a partition of $\mathcal{S}_{2}$ such that, for $s, t \in\{u, v\}, X_{s t}$ be 2-separation in $\mathcal{S}_{2}$ incident to $s_{1}$ in $\left(G_{1}^{\prime}, \Sigma_{1}\right)$ and incident to $t_{2}$ in $\left(G_{2}^{\prime}, \Sigma_{2}\right)$. Since $\left|\mathcal{S}_{2}\right| \geq 13$, without loss of generality, $\left|X_{u u}\right| \geq 4$. By Lemma 3.4.9, $X_{u u}$ is a w-star of $W_{f l i p}\left[\left(G_{1}^{\prime}, \Sigma_{1}\right), X_{v u} \cup X_{v v}\right]$ with center $u_{1}$ and a w-star of $W_{f l i p}\left[\left(G_{2}^{\prime}, \Sigma_{2}\right), X_{u v}\right]$ with a center $u_{2}$. Let $A$ be a set of vertices in the boundary of $X_{u u}$ which is not $u_{1}$.

Case 1. $|A| \geq 3$.

Let $a_{1}, a_{2}, a_{3} \in A$ and let $X_{i}$ be a 2-separation where $\mathcal{B}_{G_{1}^{\prime}}\left(X_{i}\right)$ for $i=1,2,3$. By Remark 3.4.10, $G_{1}\left[X_{1} \cup X_{2} \cup X_{3}\right]$ and $G_{1}^{\prime}\left[X_{1} \cup X_{2} \cup X_{3}\right]$ are isomorphic since every 2-separations in $\mathcal{S}_{1}$ does not contain $u_{1}, v_{1}$ at the boundary. Since $M$ is 3-connected and each $X_{i}$ is minimal, $G_{1}^{\prime}\left[X_{i}\right]$ contains an odd circuit $C_{i}$ of $G^{\prime}$. Since $u_{1}, v_{1}$ is a blocking pair of $G_{1}, C_{i}$ contains $u_{1}$ and avoids $a_{i}$ in $G_{1}^{\prime}$. Thus, $C_{1}, C_{2}, C_{3}$ are pairwise disjoint in $G_{2}^{\prime}$. By Remark 3.4.10, $C_{1}, C_{2}, C_{3}$ are pairwise disjoint in $G_{2}$ since every 2-separations in $\mathcal{S}_{3}$ does not contain $u_{2}, v_{2}$ at the boundary. Thus, $G_{2}$ has no blocking pair, giving a contradiction.

Case 1. $|A| \leq 2$.

There exists $a \in A$ such that there are at least three 2-separations $X_{1}, X_{2}, X_{3}$ in $\mathcal{S}_{2}$ with $\left\{u_{1}, a\right\}$ as boundary vertices. By Remark 3.4.10, $G_{1}\left[X_{1} \cup X_{2} \cup X_{3}\right]$ and $G_{1}^{\prime}\left[X_{1} \cup X_{2} \cup X_{3}\right]$ are isomorphic since every 2-separations in $\mathcal{S}_{1}$ does not contain $u_{1}, v_{1}$ at the boundary. Since $M$ is 3-connected and each $X_{i}$ is minimal, $G_{1}^{\prime}\left[X_{i}\right]$ contains an odd circuit $C_{i}$ of $G^{\prime}$. Since $u_{1}, v_{1}$ is a blocking pair of $G_{1}, C_{i}$ contains $u_{1}$ and avoids $a$ in $G_{1}^{\prime}$. Thus, all of $C_{1}, C_{2}, C_{3}$ have a common vertex and avoid $u_{2}$ in $G_{2}^{\prime}$. By Remark 3.4.10, $C_{1}, C_{2}, C_{3}$ have a common vertex $w$ and avoid $u_{2}$ in $G_{2}$ since every 2-separations in $\mathcal{S}_{3}$ does not contain $u_{2}, v_{2}$ at the boundary. Since $\left\{u_{2}, v_{2}\right\}$ is a blocking pair of $G_{2}, w=v_{2}$. Thus, $\left(H_{2}, T_{2}\right)$ has an ordinary anemone, giving a contradiction.

\section{Extended BP-representations with small gap}

In this section, we will prove following Lemma 3.4.12 stating that if we have large number of extended $T_{4}$-representations with pairwise small gap, then at least one of them has an ordinary 
anemone.

Lemma 3.4.12. Let $M$ be a 3-connected p-cographic matroid which is not cographic. For $i \in$ $[k]$, let $\left(H_{i}, T_{i}, P_{i}\right)$ be an extended $T_{4}$-representation for $M$. Suppose that for $i, j \in[k],\left(H_{i}, T_{i}\right.$, $\left.P_{i}\right),\left(H_{j}, T_{j}, P_{j}\right)$ are homologous. For $i \in[k]$, let $R_{i}=\left(G_{i}, \Sigma_{i},\left\{u_{i}, v_{i}\right\}\right)$ be an extended BPrepresentation obtained by folding $\left(H_{i}, T_{i}, P_{i}\right)$. Suppose that for $i, j \in[k], \operatorname{gap}\left(G_{i}, G_{j}\right) \leq 10$. Then either $\left(H_{i}, T_{i}\right)$ has an ordinary anemone for some $i \in[k]$ or $k=O\left(|E(M)|^{14}\right)$.

To prove Lemma 3.4.12, we need following lemmas. We postpone the proof for Lemma 3.4.13 until the end of this section.

Lemma 3.4.13. Let $\left(G_{1}, \Sigma_{1},\left\{u_{1}, v_{1}\right\}\right), \cdots,\left(G_{k}, \Sigma_{k},\left\{u_{k}, v_{k}\right\}\right)$ be extended BP-representations that are equivalent. Suppose for any $i, j \in[k], \operatorname{gap}\left(G_{i}, G_{j}\right) \leq 10$. Let $M=$ ecycle $\left(G_{1}, \Sigma_{1}\right)$. Then either $G_{i}=G_{j}$ for some $i \neq j$ or $k=O\left(|E(M)|^{12}\right)$.

Lemma 3.4.14. Let $M$ be a 3-connected p-cographic matroid which is not cographic. Let $\left(H_{1}, T_{1}, P_{1}\right) \ldots,\left(H_{k}, T_{k}, P_{k}\right)$ be extended $T_{4}$-representations that are pairwise homologous and let $\left(G_{i}, \Sigma_{i},\left\{u_{i}, v_{i}\right\}\right)$ be an extended BP-representation obtained by folding $\left(H_{i}, T_{i}, P_{i}\right)$. Suppose there exists a graph $G$ such that for every $i \in[k], G=G_{i}$. Then either $\left(H_{i}, T_{i}\right)$ has an ordinary anemone for some $i \in[k]$ or $k=O\left(|E(M)|^{2}\right)$.

Proof. Suppose that $k \neq O\left(|E(M)|^{2}\right)$. Let $m$ be the maximum number of extended BPrepresentations with the same blocking pair $\{u, v\}$ and let $I \subseteq[k]$ be the set of indices of these extended BP-representations. Note that there are at most $|V(G)|^{2}$ choices for a blocking pair $\{u, v\}$ in $G$. Thus, $m \neq O(1)$. Let $E_{0}$ be a set of edges of $G$ that have both ends in $u, v$. Let $C_{1}, \ldots, C_{l}$ be components of $G \backslash\{u, v\}$. For $a \in[l]$, let $P_{a}=G\left[V\left(C_{a}\right) \cup\{u, v\}\right]$. By 3 -connectivity of $M$, for every $a \in[l], \mathcal{B}_{G}\left(P_{a}\right)=\{u, v\}$. For $i, j \in I$, since $\left(G, \Sigma_{i}\right)$ and $\left(G, \Sigma_{j}\right)$ are equivalent, $D:=\Sigma_{i} \triangle \Sigma_{j}$ is a cut of $G$. Note that $\Sigma_{i} \cup \Sigma_{j} \subseteq \delta(u) \cup \delta(v)$. Thus, $D$ is a cut of $G$ generated by $\delta(u), \delta(v)$ and $\delta\left(P_{1}\right) \ldots, \delta\left(P_{l}\right)$. Thus $l \geq 3$ and $\left(E_{0},\left\{P_{1}, \ldots, P_{l}\right\}\right)$ is a graphic anemone. By Lemma 3.4.11, for $i \in I,\left(H_{i}, T_{i}\right)$ has an ordinary anemone.

Before we prove Lemma 3.4.13, we need following lemmas.

Lemma 3.4.15. Let $\left(H_{1}, T_{1}, P_{1}\right),\left(H_{2}, T_{2}, P_{2}\right)$ be extended $T_{4}$-representations that are homologous and for $i=1,2$, let $R_{i}=\left(G_{i}, \Sigma_{i},\left\{u_{i}, v_{i}\right\}\right)$ be extended BP-representations obtained by folding $\left(H_{i}, T_{i}, P_{i}\right)$. Then there exists $\left(H_{2}^{\prime}, T_{2}^{\prime}, P_{2}^{\prime}\right)$ and its folding $R_{2}^{\prime}=\left(G_{2}^{\prime}, \Sigma_{2}^{\prime},\left\{u_{2}^{\prime}, v_{2}^{\prime}\right\}\right)$ such that

(1) $\left(H_{2}^{\prime}, T_{2}^{\prime}, P_{2}^{\prime}\right)$ and $\left(H_{2}, T_{2}, P_{2}\right)$ are equivalent. 
(2) $\left(H_{2}^{\prime}, T_{2}^{\prime}, P_{2}^{\prime}\right)$ and $\left(H_{1}, T_{1}, P_{1}\right)$ are homologous.

(3) there exists a nice $w$-sequence $\left(\mathcal{S}_{1}, \mathcal{S}_{2}, \mathcal{S}_{3}\right)$ from $R_{1}$ to $R_{2}^{\prime}$ such that $\left|\mathcal{S}_{2}\right|=\operatorname{gap}\left(R_{1}, R_{2}\right)=$ $\operatorname{gap}\left(R_{1}, R_{2}^{\prime}\right)$ and $\left|\mathcal{S}_{3}\right|=0$.

Proof. Let $\left(\mathcal{S}_{1}^{\prime}, \mathcal{S}_{2}^{\prime}, \mathcal{S}_{3}^{\prime}\right)$ be a nice w-sequence from $R_{1}$ to $R_{2}$ where $\left|\mathcal{S}_{2}^{\prime}\right|=\operatorname{gap}\left(R_{1}, R_{2}\right)$ and let $\mathcal{S}_{3}^{\prime}=\left(X_{1}, \ldots, X_{k}\right)$ and let $\overline{\mathcal{S}}_{3}^{\prime}$ be a w-sequence of $G_{2}$ obtained by reverse the order of $\mathcal{S}_{3}$. Since $X_{i} \cap\left\{u_{2}, v_{2}\right\}=\emptyset$ for all $i \in[k], \overline{\mathcal{S}}_{3}^{\prime}$ is a w-sequence of $H_{2}$. Let $\left(H_{2}^{\prime}, T_{2}^{\prime}\right)=W_{\text {flip }}\left[\left(H_{2}, T_{2}\right), \overline{\mathcal{S}}_{3}^{\prime}\right]$. Since $X_{i} \cap\left\{u_{2}, v_{2}\right\}=\emptyset, \mathcal{I}_{H_{2}}\left(X_{i}\right) \cap T_{2}$ has even size. Thus, $T_{2}^{\prime}=T_{2}$ and $\left(H_{2}^{\prime}, T_{2}, P_{2}\right),\left(H_{2}, T_{2}, P_{2}\right)$ are equivalent. Let $R_{2}^{\prime}$ be a extended BP-representation obtained by folding $\left(H_{2}^{\prime}, T_{2}, P_{2}\right)$. Then, $\left(\mathcal{S}_{1}^{\prime}, \mathcal{S}_{2}^{\prime}, \emptyset\right)$ is a nice w-sequence from $R_{1}$ to $R_{2}^{\prime}$. Thus, $\left(H_{2}^{\prime}, T_{2}^{\prime}, P_{2}^{\prime}\right)$ and $\left(H_{1}, T_{1}, P_{1}\right)$ are homologous. For the last part, $\left|\mathcal{S}_{2}^{\prime}\right|=\operatorname{gap}\left(R_{1}, R_{2}^{\prime}\right)$, otherwise $\operatorname{gap}\left(R_{1}, R_{2}\right)$ can be smaller.

We may assume that $\left(H_{2}^{\prime}, T_{2}, P_{2}\right)$ is chosen with minimum $\left|\mathcal{S}_{1}\right|$. In this case, we call $\left(H_{2}^{\prime}\right.$, $\left.T_{2}, P_{2}\right)$ a alternative of $\left(H_{2}, T_{2}, P_{2}\right)$ for $\left(H_{1}, T_{1}, P_{1}\right)$.

Lemma 3.4.16. Let $M$ be a 3-connected p-cographic matroid that is not cographic. Let $\left(H_{1}, T_{1}\right.$, $\left.P_{1}\right),\left(H_{2}^{\prime}, T_{2}, P_{2}\right)$ be extended $T_{4}$-representations for $M$ that are homologous and let $\left(H_{2}, T_{2}, P_{2}\right)$ be an alternative of $\left(H_{2}^{\prime}, T_{2}, P_{2}\right)$ for $\left(H_{1}, T_{1}, P_{1}\right)$ For $i \in[2]$, let $R_{i}=\left(G_{i}, \Sigma_{i},\left\{u_{i}, v_{i}\right\}\right)$ be extended BP-representations obtained by folding $\left(H_{i}, T_{i}, P_{i}\right)$. Then $\mathcal{S}_{1}$ is non-crossing. Moreover, there exists a set $\mathcal{S}$ only dependent of $\left(H_{1}, T_{1}, P_{1}\right)$ such that $|\mathcal{S}| \leq 16$ and $\mathcal{S}_{1} \in \mathcal{S}$ for any choice of $\left(H_{2}^{\prime}, T_{2}, P_{2}\right)$.

Proof. Let $\mathcal{X}=\left\{X_{1}, \ldots, X_{k}\right\}$ be the set of all minimal 2-separations of $G_{1}$ avoiding $u_{1}, v_{1}$. Note that $\mathcal{S}_{1} \subseteq \mathcal{X}$.

Claim 1. $\mathcal{X}$ is non-crossing.

Proof. Suppose $\mathcal{X}$ is crossing. Let $\left(X_{i}, \bar{X}_{i}\right),\left(X_{j}, \bar{X}_{j}\right)$ be the pair of 2-separations in $\mathcal{X}$. Since $X_{i}, X_{j}$ are minimal, all of $X_{i} \cap X_{j}, \bar{X}_{i} \cap X_{j}, X_{i} \cap \bar{X}_{j}, \bar{X}_{i} \cap \bar{X}_{j}$ are either a 2-separation in $G_{1}$ or a set of one edge. By 3-connectivity, at least three of them contains at least two edges and each of them contains an odd cycle. Since they cannot contain $u_{1}$ or $v_{1}$ in their boundary, there is no blocking pair. Contradiction.

The first part of the lemma follows from Claim 1 since $\mathcal{S}_{1} \subseteq \mathcal{X}$.

Claim 2. We may assume $X_{1} \subseteq \cdots \subseteq X_{k}$. 
Proof. By 3-connectivity of $M$, for $i \in[k]$, each of $X_{i}$ and $\bar{X}_{i}$ contains one vertex of $\left\{u_{1}, v_{1}\right\}$ in its interior. We may assume $u_{1} \in \mathcal{I}_{G_{1}}\left(X_{i}\right)$ for all $i \in[k]$. For distinct $i, j \in[k], X_{i}, X_{j}$ are non-crossing, so $X_{i} \subseteq X_{j}$ or $X_{j} \subseteq X_{i}$. Since $\mathcal{X}$ is non-crossing, we can rearrange $\mathcal{X}$ such that $X_{1} \subseteq \cdots \subseteq X_{k}$.

If $|\mathcal{X}| \leq 2$, then there are only four possibilities for $\mathcal{S}_{1}$. Thus, we may assume that $|\mathcal{X}| \geq 3$.

Claim 3. There is no $X \in \mathcal{S}_{2}$ such that $X, X_{2}$ are crossing.

Proof. Suppose there exists $X \in \mathcal{S}_{2}$ such that $X, X_{2}$ are crossing. We may assume $u_{1} \in \mathcal{B}_{G_{1}}(X)$. Since $u_{1} \in \mathcal{I}_{G_{1}}\left(X_{1}\right)$ and $X_{1} \subseteq X_{2}, X_{1}, X$ are crossing. Since $X, X_{1}, X_{2}$ are minimal, all of $X \cap X_{1}, \bar{X} \cap X_{1}, X \cap\left(X_{2}-X_{1}\right), \bar{X} \cap\left(X_{2}-X_{1}\right), X \cap \bar{X}_{2}, \bar{X} \cap \bar{X}_{2}$ are either a 2-separation of $G_{1}$, an empty set or a set of one edge. Since $X$ crosses with $X_{1}$ and $X_{2}$ respectively, $X \cap X_{1}$, $\bar{X} \cap X_{1}, X \cap \bar{X}_{2}, \bar{X} \cap \bar{X}_{2}$ are not empty and at least one of $X \cap\left(X_{2}-X_{1}\right), \bar{X} \cap\left(X_{2}-X_{1}\right)$ is not empty, otherwise $X_{1}=X_{2}$. By 3-connectivity of $M$, at least 4 of them contain at least two edges and each of them contains an odd cycle of $\left(G_{1}, \Sigma_{1}\right)$. Thus, $\mathcal{B}_{G_{1}}(X)=\left\{u_{1}, v_{1}\right\}$. Thus, $X$ is crossing with each element of $\mathcal{S}_{2}$. By the similar argument above, at least 6 of $X \cap X_{1}, \bar{X} \cap X_{1}$, $X \cap\left(X_{2}-X_{1}\right), \bar{X} \cap\left(X_{2}-X_{1}\right), X \cap\left(X_{3}-X_{2}\right), \bar{X} \cap\left(X_{3}-X_{2}\right), X \cap \bar{X}_{3}, \bar{X} \cap \bar{X}_{3}$ contain an odd cycle of $\left(G_{1}, \Sigma_{1}\right)$ and then there is no blocking pair. Contradiction.

It follows from Claim 3 that for each $X \in \mathcal{S}_{2}, X$ crosses with none of $X_{2}, \ldots, X_{k-1}$. Thus for $i=2, \ldots, k-1, X_{i}$ is a 2-separation of $G_{2}$. By the construction of alternative representation, $X_{i}$ is not a 2-separation of $H_{2}$. Thus, when we unfold $\left(G_{2}, \Sigma_{2}\right)$ to $\left(H_{2}, T_{2}\right)$, at least one vertex of $\mathcal{B}_{G_{2}}\left(X_{i}\right)$ is splitted, namely, $\mathcal{B}_{G_{2}}\left(X_{i}\right)$ contains at least one of $u_{2}, v_{2}$. We may assume that $u_{2} \in \mathcal{B}_{G_{2}}\left(X_{i}\right)$ for some $2 \leq i \leq k-1$.

Claim 4. For $2 \leq j \leq k-1, j \neq i, u_{2} \in \mathcal{B}_{G_{2}}\left(X_{j}\right)$.

Proof. Suppose $v_{2} \in \mathcal{B}_{G_{2}}\left(X_{j}\right)$. We may assume $j<i$. Since $X_{i}$ is not a 2-separation after unfolding and $M$ is 3-connected, there exists an odd circuit $C$ containing $u_{2}$ and avoiding $v_{2}$ such that $C \subseteq X_{i}$. Since $C$ avoids $v_{2}, C \subseteq\left(X_{i}-X_{j}\right)$. Then $C$ avoids $u_{1}, v_{1}$ in $G_{1}$. Contradiction. $\diamond$

Since for all $2 \leq i \leq k-1, \mathcal{B}_{G_{2}}\left(X_{i}\right)$ share a vertex in common, there are fixed choices of $X_{3}, \ldots, X_{k-2}$ for $\mathcal{S}_{1}$. Thus, there are only constantly many $\mathcal{S}_{1}$ for all $\left(H_{2}^{\prime}, T_{2}, P_{2}\right)$.

Now, we are ready to prove Lemma 3.4.13 and Lemma 3.4.12. 
Proof of Lemma 3.4.13. Let us fix $\left(G_{1}, \Sigma_{1},\left\{u_{1}, v_{1}\right\}\right.$ and for $i=2, \ldots, k$, let $\left(G_{i}^{\prime}, \Sigma_{i}^{\prime},\left\{u_{i}^{\prime}, v_{i}^{\prime}\right\}\right.$ be alternatives of $\left(G_{i}, \Sigma_{i},\left\{u_{i}, v_{i}\right\}\right.$. Let $\left(\mathcal{S}_{1}^{\prime}, \mathcal{S}_{2}, \emptyset\right)$ be a nice sequence from $G_{1}$ to $G_{i}^{\prime}$. By Lemma 3.4.16, $\mathcal{S}_{1}$ is non-crossing Whitney flips and $\left|\mathcal{S}_{1}\right| \leq 2$. Thus, there are $O\left(|E(M)|^{2}\right)$ choices for $\mathcal{S}_{1}^{\prime}$. Let $K_{i}=W_{f l i p}\left[G_{i}^{\prime}, \mathcal{S}_{1}^{\prime}\right]$. Then $\mathcal{S}_{2}$ is non-crossing Whitney flips of $K_{i}$ and $\left|\mathcal{S}_{2}\right| \leq 9$. Thus, there are $O\left(|E(M)|^{10}\right)$ choices for $\mathcal{S}_{2}$. Thus, $k=O\left(|E(M)|^{12}\right)$.

Proof of Lemma 3.4.12. Suppose that $k \neq O\left(|E(M)|^{14}\right)$. Let $m$ be the maximum number of extended BP-representations with the same graph $G$ and let $I$ be the set of indices of these extended BP-representations. By Lemma 3.4.13, $m \neq O\left(\mid E(M)^{2}\right)$. By Lemma 3.4.14, there exists $i \in[k]$ such that $\left(H_{i}, T_{i}\right)$ has an ordinary anemone.

\section{The proof of Lemma 3.4.2}

We now prove Theorem 3.4.2.

Proof of Theorem 3.4.2. For $i \in[k]$, let $R_{i}=\left(H_{i}, T_{i}, P_{i}\right)$ be an extended $T_{4}$-representation for $N$ that is a representative for $\mathcal{F}_{i}$. By Lemma 3.4.6, $R_{1}, \ldots, R_{k}$ are homologous. For $i \in[k]$, let $S_{i}=\left(G_{i}, \Sigma_{i},\left\{u_{i}, v_{i}\right\}\right)$ be an extended $B P$-representation obtained by folding $R_{i}$. Let $m$ be the maximum gap among $S_{1}, \ldots, S_{k}$. Let $i, j \in[k]$ be the indices such that $\operatorname{gap}\left(S_{i}, S_{j}\right)=m$. If $m>10$, then by Lemma 3.4.8, then at least one of $\left(H_{i}, T_{i}\right)$ and $\left(H_{j}, T_{j}\right)$ has an ordinary anemone, giving a contradiction. If $m \leq 10$, then by Lemma 3.4.12, $k=O\left(|E(M)|^{14}\right)$. 


\section{References}

[1] W. H. Cunningham and J. Edmonds. A combinatorial decomposition theory. Canad. J. Math., 32:734-765, 1980.

[2] J. Geelen, P. Hliněný and G. Whittle. Bridging separations in matroids. SIAM J. Discrete Math., 18:638-646, 2005.

[3] A.M.H. Gerards. On tutte's characterization of graphic matroids - a graphic proof. J. Graph Theory, 20:351-359, 1995.

[4] J. Geelen, A. M. H. Gerards and A. Kapoor. The excluded minors for $g f(4)$-representable matroids. J. Combin. Theory Ser. B, 79:247-299, 2000.

[5] B. Guenin. personal conversation. 2016.

[6] B. Guenin and I. Pivotto. Stabilizer theorems for even cut matroids. Submitted.

[7] A. J. Hoffman. Some recent applications of the theory of linear inequalities to extremal combinatorial analysis. In Combinatorial Analysis (Proceedings of Symposia in Applied Mathematics, Vol. X) (R. Bellman and M. Hall, Jr., eds.), pages 113-127. American Mathematical Society, Providence, Rhode Island, 1960.

[8] G. Whittle J. Geelen, A.M.H. Gerards. in preparation.

[9] J. Oxley. Matroid Theory. Oxford University Press, 1992.

[10] B. Guenin, I. Pivotto and P. Wollan. Stabilizer theorems for even cycle matroids. J. Combin. Theory Ser. B, 118:44-75, 2016.

[11] I. Pivotto. Even cycle and even cut matroids. Ph.D thesis, University of Waterloo, 2011.

[12] J. Oxley, C. Semple and G. Whittle. The structure of the 3-separations of 3-connected matroids. J. Combin. Theory Ser. B, 92:257-293, 2004. 
[13] P.D. Seymour. A note on the production of matroid minors. J. Combin. Theory Ser. B, 22:289-295, 1977.

[14] P.D. Seymour. On tutte's characterization of graphic matroids. In In Combinatorics 79. Part I(eds. M. Deza and I. G. Rosenberg)., volume 8, pages 83-90. Ann. Discrete Math., North-Holland, Amsterdam, 1980.

[15] W. T. Tutte. An algorithm for determining whether a given binary matroid is graphic. Proc. Amer. Math. Soc., 11:905-917, 1960.

[16] W. T. Tutte. Connectivity in matroids. Canad. J. Math., 18:1301-1324, 1966.

[17] H. Whitney. 2-isomorphic graphs. Amer. J. Math., 55:245-254, 1933. 